\title{
Renormalization of Vector Fields
}

\author{
Hans Koch ${ }^{2}$
}

\begin{abstract}
These notes cover some of the recent developments in the renormalization of quasiperiodic flows. This includes skew flows over tori, Hamiltonian flows, and other flows on $\mathbb{T}^{d} \times \mathbb{R}^{\ell}$. After stating some of the problems and describing alternative approaches, we focus on the definition and basic properties of a single renormalization step. A second part deals with the construction of conjugacies and invariant tori, including shearless tori, and non-differentiable tori for critical Hamiltonians. Then we discuss properties related to the spectrum of the linearized renormalization transformation, such as the accumulation rates for sequences of closed orbits. The last part describes extensions from "self-similar" to Diophantine rotation vectors. This involves sequences of renormalization transformations that are related to continued fractions expansions in one and more dimensions. Whenever appropriate, the discussion of details is restricted to special cases where inessential technical complications can be avoided.
\end{abstract}

Expanded notes from a mini-course given at the Fields Institute in Toronto, Canada, November 2005

2 Department of Mathematics, The University of Texas at Austin, 1 University Station C1200, Austin, TX $78712-0257$ 
Content

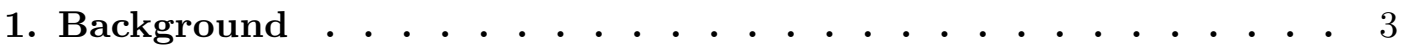

1.1 Invariant tori . . . . . . . . . . . . . . . . . . . . . . . . . . 3

1.2 Two direct approaches . . . . . . . . . . . . . . . . . . . . . . 3

1.3 Hamiltonians . . . . . . . . . . . . . . . . . . . . . . . . . . 4

$1.4 \mathrm{KAM}$ theory . . . . . . . . . . . . . . . . . . . . . . 5

1.5 Scales . . . . . . . . . . . . . . . . . . . . 6

1.6 Breakup of invariant tori . . . . . . . . . . . . . . . . . . . . . 7

2. Renormalization of flows . . . . . . . . . . . . . . 9

2.1 Hamiltonian systems . . . . . . . . . . . . . . . . . . . . . . . 9

2.2 Resonant and nonresonant Hamiltonians . . . . . . . . . . . . . . 11

2.3 The change of variables $\mathcal{U}_{H}$. $\quad . \quad$. . . . . . . . . . . . . . . . . . . . . . . . 13

2.4 Other vector fields . . . . . . . . . . . . . . . . . . . . . . . 14

2.5 Skew systems . . . . . . . . . . . . . . . . . . . . 15

3. A single renormalization group step . . . . . . . . . . . 17

3.1 Skew systems: definitions . . . . . . . . . . . . . . . . . . . . . 17

3.2 Skew systems: estimates . . . . . . . . . . . . . . . . . . . . 18

3.3 More general vector fields . . . . . . . . . . . . . . . . . . . 20

3.4 A general elimination procedure . . . . . . . . . . . . . . . . . 21

4. A nontrivial RG fixed point . . . . . . . . . . . . . . 24

4.1 Observations and result . . . . . . . . . . . . . . . . . . . . . . 24

4.2 Strategy of proof . . . . . . . . . . . . . . . . . . . 26

4.3 Non-twist flows . . . . . . . . . . . . . . . . . . . . . . . . . 28

5. Invariant tori . . . . . . . . . . . . . . . . . . . . . . 31

5.1 Some ideas and results . . . . . . . . . . . . . . . . . . . . . . 31

5.2 Renormalization of invariant tori . . . . . . . . . . . . . . . . 34

5.3 Existence . . . . . . . . . . . . . . . . . . . . . . . . . 36

5.4 Critical invariant tori . . . . . . . . . . . . . . . . . . . . . 38

5.5 Shearless tori . . . . . . . . . . . . . . . . . . . . . 40

6. Scaling . . . . . . . . . . . . . . . . . . . 43

6.1 Spectrum of the linearized RG transformation . . . . . . . . . . . . 43

6.2 Accumulation of periodic orbits . . . . . . . . . . . . . . . . . . 44

6.3 Choice of the manifold $\Sigma_{0} \quad$. . . . . . . . . . . . . . . . . . . . . . . . 47

6.4 The manifolds $\Sigma_{n}$ and orbits $\gamma_{n}$. . . . . . . . . . . . . . . . . . . . . . . 49

7. Sequences of RG transformations . . . . . . . . . . . 51

7.1 Diophantine and Brjuno numbers . . . . . . . . . . . . . . . . . 51

7.2 Multidimensional continued fractions . . . . . . . . . . . . . . . 52

7.3 Composing different RG transformations . . . . . . . . . . . . . . 54

7.4 An invariant manifold theorem . . . . . . . . . . . . . . . . . . 56

8. Reduction of skew flows . . . . . . . . . . . . . 58

8.1 A general result . . . . . . . . . . . . . . . . . . . . . . . 58

8.2 The stable manifold . . . . . . . . . . . . . . . . . . . . . . . 59

8.3 Conjugacy to a linear flow . . . . . . . . . . . . . . . . . . . . 60

8.4 The special case $\mathfrak{G}=\mathrm{SL}(2, \mathbb{R}) \quad$. . . . . . . . . . . . . . . . . . . . . . . 62

8.5 Excluding hyperbolicity . . . . . . . . . . . . . . . . . . . . . 64

References . . . . . . . . . . . . . . . . . 65 


\section{Background}

\subsection{Disclaimer}

This review is primarily about methods and ideas. It does not intend to give a comprehensive list of theorems on invariant tori, conjugacies, bifurcations, and other topics covered. The main focus is on renormalization group methods for Hamiltonian and other vector fields. And more specifically, on methods that that implement renormalization as a dynamical system on a space of Hamiltonians or vector fields. Much of the discussion is restricted to problems that I have worked on myself, which is not meant to imply that other work is less important.

\subsection{Invariant tori}

The general goal is to describe certain asymptotic behavior, like quasiperiodicity, for continuous-time dynamical systems

$$
\dot{u}=X(u) \text {. }
$$

Here, $X$ is a vector field on some manifold $\mathcal{M}$. The flow $\Phi_{X}$ associated with $X$ is defined by $\Phi_{X}^{t}\left(u_{0}\right)=u(t)$, where $u$ is the solution of (1.1) with initial condition $u(0)=u_{0}$.

In all cases discussed here, $\mathcal{M}$ will be a product of the $d$-torus $\mathbb{T}^{d}$ with some other manifold $B$. Most of the problems considered involve either invariant tori or conjugacies. By an invariant torus with rotation vector $\omega \in \mathbb{R}^{d}$, we mean a map $\Gamma: \mathbb{T}^{d} \rightarrow \mathcal{M}$ that is locally one-to-one and satisfies

$$
\Gamma \circ \Psi^{t}=\Phi_{X}^{t} \circ \Gamma, \quad \Psi^{t}(q)=q+t \omega .
$$

In other words, $\Gamma$ defines a semi-conjugacy between a restriction (to the range of $\Gamma$ ) of the original flow $\Phi_{X}$ and the linear flow $\Psi$ on the torus. Some true conjugacies $\Phi_{X}=U \circ \Phi_{Z} \circ U^{-1}$ will be discussed as well, where it is possible to conjugate $\Phi_{X}$ to the flow for a trivial vector field $Z$ on all of $\mathcal{M}$.

In order to see some of the difficulties involved in solving equation (1.2), it is useful to look at the differentiated version

$$
\omega \cdot \nabla \Gamma=X \circ \Gamma .
$$

One of the problems is that the differential operator $\omega \cdot \nabla$ is not easily invertible. Its spectrum, if nontrivial, accumulates at zero in a way that depends on the arithmetic properties or the rotation vector $\omega$.

\subsection{Two direct approaches}

One way of trying to solve equation (1.3) is by perturbing about an approximate solution $\Gamma_{0}$. Substituting $\Gamma=\Gamma_{0}+\gamma$ and expanding $X \circ\left(\Gamma_{0}+\gamma\right)$ in powers of $\gamma$, we obtain an equation of the form

$$
\gamma=\gamma_{0}+W(\gamma), \quad W(\gamma)=\sum_{m>1} \mathcal{D}^{-1} W_{n}(\gamma)^{m}
$$


where $\mathcal{D}=\omega \cdot \nabla-D X \circ \Gamma_{0}$. Iterating the map $\gamma \mapsto \gamma_{0}+W(\gamma)$ yields a formal power series for the torus, also referred to as Lindstedt series. This series is in general highly divergent, but there are nontrivial situations where resummation techniques can be used to obtain $\gamma$ from its Lindstedt series [39].

Interestingly, the problems that one encounters are similarly to those found for Feynman graph expansions in quantum field theory. It is these expansions that lead to the development of renormalization methods [118]. The divergencies can be associated with different "scales" in the problem, and by re-normalizing the expansion parameters appropriately, the divergencies at any given scale cancel. Applications of renormalization ideas from quantum field theory to the resummation problem for Lindstedt series can be found e.g. in $[51,57,58,59]$. In this context, renormalization can be viewed as a method for dealing with combinatorial problems and cancellations in certain highly nontrivial perturbation expansions.

Equation (1.3) also has a vague resemblance to field equations in quantum field theory. In some special cases, it is possible to make this connection more precise and write (1.3) as the Euler-Lagrange equation for some functional $\gamma \mapsto \mathcal{L}_{1}(\gamma)$. The modern way of analyzing such fields is via functional integrals. Expanding these integrals in powers of small coupling constants yields the above-mentioned Feynman graphs. (Integrals of the same type also appear in statistical mechanics, where there are usually no small parameters.) The approach taken in non-perturbative renormalization is to perform the integration one scale at a time, transforming a Lagrangian $\mathcal{L}_{k}$ at scale $k$ to a new Lagrangian $\mathcal{L}_{k+1}$ at scale $k+1$, making the map $\mathcal{L}_{k} \mapsto \mathcal{L}_{k+1}$ a dynamical system, if possible. Inspired by this approach, Bricmont et. al. have devised a renormalization scheme that applies to the problem of constructing invariant tori [8]. The formalism itself is non-perturbative, but in practice, the analysis can be carried out only for $X$ close to constant, where $\gamma$ is small. Similar ideas have also been applied successfully to the study of PDEs [9].

In the context described here, renormalization can be viewed as a procedure for solving certain difficult "scale free" problems iteratively, one scale at a time.

\subsection{Hamiltonians}

The renormalization group approach that we will focus on later is much closer to KAM theory than to the approaches sketched above. In order to simplify the discussion, we will restrict our attention to Hamiltonian flows. Consider $\mathcal{M}=\mathbb{T}^{d} \times B$, where $B$ is some open neighborhood of the origin in $\mathbb{R}^{d}$. A Hamiltonian vector field in action-angle variables is of the form $X=\mathbb{J} \nabla H$, with $\mathbb{J}=\left[\begin{array}{cc}0 & I \\ -I & 0\end{array}\right]$, where $H$ is the corresponding Hamiltonian, a differentiable function on $\mathcal{M}$. In other words, the equation $\dot{u}=X(u)$, with $u=(q, p)$, can be written as

$$
\dot{q}=\nabla_{p} H, \quad \dot{p}=-\nabla_{q} H .
$$

The corresponding flow will be denoted by $\Phi_{H}$.

Some basic facts and notation: $H$ is invariant under the flow. The maps $\Phi_{H}^{t}$ are symplectic, in the sense that they preserve the symplectic form $\sum d q_{j} \wedge d p_{j}$. If $U$ is any symplectic diffeomorphism of $\mathcal{M}$, then the pushforward of $X$ under $U$ is again a Hamiltonian vector field, with Hamiltonian $H \circ U$. Furthermore, $U$ preserves the Poisson bracket $\{f, g\}=\nabla_{1} f \cdot \nabla_{2} g-\nabla_{2} f \cdot \nabla_{1} g$. 
A change of coordinates $\left(q^{\prime}, p^{\prime}\right)=U(q, p)$ is canonical if and only if the one-form $p^{\prime} \cdot d q^{\prime}-p \cdot d q$ is closed. Locally, this one-form can be written as the differential of some function, which we will write as $p^{\prime} \cdot q^{\prime}-\phi$. We will only be interested in cases where $\phi$ is defined globally, as a function of $q$ and $p^{\prime}$. In this case, $\phi$ will be referred to as the generating function of $U$. It satisfies $q^{\prime}=\nabla_{2} \phi\left(q, p^{\prime}\right)$ and $p=\nabla_{1} \phi\left(q, p^{\prime}\right)$. In particular, if

$$
U=\mathrm{I}+u, \quad u(q, p)=(Q(q, p), P(q, p)),
$$

then we have

$$
Q(q, p)=\left(\nabla_{2} \phi\right)(q, p+P(q, p)), \quad P(q, p)=-\left(\nabla_{1} \phi\right)(q, p+P(q, p)) .
$$

Conversely, given $\phi$ not too large, these two equations determine a canonical transformation of the form (1.6). If $\phi$ is small, say of "size $\varepsilon$ ", then so are $P, Q$, and we have

$$
H \circ U=H\left(.+\nabla_{2} \phi, .-\nabla_{1} \phi\right)+\mathcal{O}\left(\varepsilon^{2}\right)=H+\{H, \phi\}+\mathcal{O}\left(\varepsilon^{2}\right) .
$$

\subsection{KAM theory}

KAM theory $[82,5,110,121,26]$ is concerned with with small perturbations of integrable Hamiltonians, such as

$$
K(q, p)=\omega \cdot p+\frac{1}{2}(M p) \cdot p
$$

with $\omega \in \mathbb{R}^{d}$ and $M$ a symmetric $d \times d$ matrix. The dynamics for $K$ is given by $\dot{q}=\omega+M p$ and $\dot{p}=0$. Notice that surfaces of constant $p$ are invariant tori for the flow generated by $K$, with frequency vector $w(p)=\omega+M p$.

The goal is to construct an invariant torus for $H=K+h$ with rotation vector $\omega$, by iterating the following procedure. Assuming that $h$ is small, say of "size $\varepsilon$ ", we have

$$
\begin{aligned}
(K+h) \circ U & =K+\{K, \phi\}+h+\mathcal{O}\left(\varepsilon^{2}\right) \\
& =K-\left[w \cdot \nabla_{1} \phi-h\right]+\mathcal{O}\left(\varepsilon^{2}\right) .
\end{aligned}
$$

Now we try to solve $[\ldots]=0$ near $p=0$, up to an error of order $\varepsilon^{2}$. The equation for the $\nu$-th Fourier mode of $\phi$ is

$$
i w \cdot \nu \phi_{\nu}=h_{\nu}+\mathcal{O}\left(\varepsilon^{2}\right) .
$$

So among other things, the average $h_{0}$ has to be small near $p=0$. Assuming that the matrix $M$ is nonsingular, this can be achieved by a $p$-translation, and a restriction to $p$ near zero. Assuming in addition that $\omega$ satisfies a Diophantine condition, equation (1.11) can be solved for frequencies $\nu$ that are not too large. Finally, if we also assume that $h$ is analytic, so that $h_{\nu} \rightarrow 0$ rapidly as $|\nu| \rightarrow \infty$, then the equation (1.11) can be solved for all $\nu$. Thus, the new Hamiltonian $(K+h) \circ U$ is of the form $K+g$, with $g$ of order $\varepsilon^{2}$. Now the procedure is iterated.

The KAM theorem for this situation states that, under the assumptions made above, the invariant torus with frequency vector $\omega$ persists under small perturbations of $K$. 


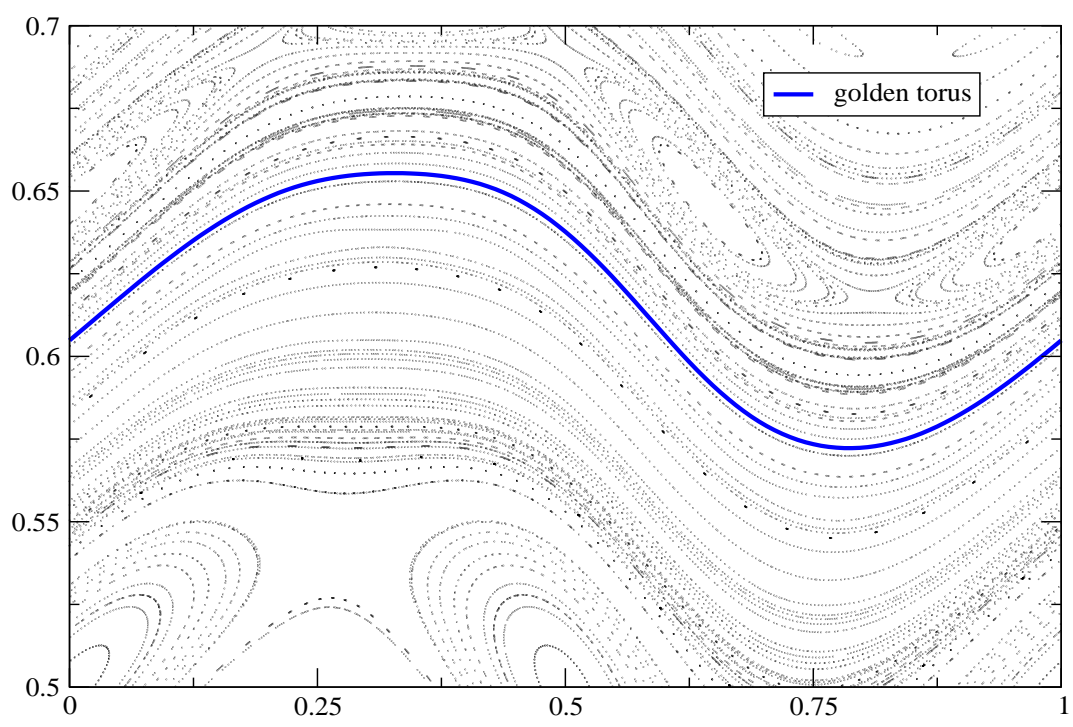

Fig. 1. Some orbits for the standard map with parameter value 0.5 [61].

Notice that the assumptions are also satisfied if $\omega$ is replaced by $c \omega$, with $c \neq 0$. The invariant tori for different values of $c$ lie on different energy surfaces. The average speed of the motion varies with $c$, but the winding numbers

$$
\lim _{t \rightarrow \infty} \frac{q_{j}(t)}{q_{d}(t)}=\frac{\omega_{j}}{\omega_{d}}
$$

do not depend on $c$. If the goal is to construct an invariant torus with fixed winding numbers, but unspecified value of $c$, then the nondegeneracy assumption on the matrix $M$ can be weakened: $M$ is allowed to have rank $d-1$, as long as the range is transversal to $\omega$. Such Hamiltonians are also referred to as isoenergetically nondegenerate.

The KAM torus for $H$ is obtained as the limit of $\Gamma_{n}=U_{1} \circ U_{2} \circ \ldots \circ U_{n}$ as $n \rightarrow \infty$, where $U_{n}$ denotes the canonical transformation used in the $n$-th step of the iteration described above. $\Gamma_{n}$ is defined on a domain $\mathbb{T}^{d} \times B_{n}$, with $\left\{B_{n}\right\}$ a sequence of smaller and smaller neighborhoods of zero. The limit $\Gamma$ yields the desired conjugacy

$$
\Gamma \circ \Phi_{K}^{t}=\Phi_{H}^{t} \circ \Gamma, \quad \text { on } \mathbb{T}^{d} \times\{0\}
$$

The KAM procedure has clearly a renormalization flavor, although some ingredients are missing, most notably the scaling. As we will see later, by modifying this algorithm appropriately, it can be made into a dynamical system. Some earlier steps in this direction were also taken in $[71,72,83]$.

\subsection{Scales}

Renormalization applies mainly to systems that involve a natural progression of scales, but that do not have any preferred scale. The effect of a renormalization operator is to shift the scales of the system. 
The best known examples in dynamical systems may be the composition operators $\mathcal{R}: F \mapsto F^{k}$ (modulo rescaling). Here, and in what follows, $F^{k}$ denotes the $k$-th iterate of a map $F$. These operators have been studied in great detail [128], after the observation of universality and scaling in one-parameter families of interval maps undergoing period doubling bifurcations $1 \rightarrow 2 \rightarrow \ldots \rightarrow 2^{n-1} \rightarrow 2^{n} \rightarrow \ldots$. The $k=2$ version of $\mathcal{R}$ lifts the inverse cascade to the space of maps, in the sense that $F \circ F$ has a period $2^{n-1}$ whenever $F$ has a period $2^{n}$.

In problems dealing with irrational rotations, the scales come from the arithmetic properties of the rotation numbers. Consider e.g. the number $\alpha=1 /(k+1 /(k+\ldots)))$, with $k$ some fixed positive integer. (For $k=1, \alpha$ is the inverse golden mean.) Its continued fraction approximants $u_{n} / v_{n}$ may be obtained as follows:

$$
\left[\begin{array}{l}
u_{n} \\
v_{n}
\end{array}\right]=T^{n}\left[\begin{array}{l}
0 \\
1
\end{array}\right], \quad T=\left[\begin{array}{ll}
0 & 1 \\
1 & k
\end{array}\right] .
$$

Consider a circle $\mathcal{C}$, defined by a strictly monotone function $C$ on $\mathbb{R}$, by identifying $C(x)$ with $x$ for any real $x$. A simple example would be $C_{0}(x)=x-1$. A map on $\mathcal{C}$ is a function $M: \mathbb{R} \rightarrow \mathbb{R}$ that commutes with $C$, and a point $x$ has rotation number $u / v$ for this map if $C^{u} \circ M^{v}(x)=x$. In this formulation, a renormalization operator that takes a pair $F=\left[\begin{array}{c}C \\ M\end{array}\right]$ with rotation number $u_{n-1} / v_{n-1}$ to a pair with rotation number $u_{n} / v_{n}$, is given by

$$
\mathcal{R}:\left[\begin{array}{c}
C \\
M
\end{array}\right] \mapsto\left[\begin{array}{lll}
C^{0} \circ M^{1} \\
C^{1} \circ M^{k}
\end{array}\right] \quad \text { (modulo rescaling) } .
$$

The pair $F_{0}=\left[\begin{array}{l}C_{0} \\ M_{0}\end{array}\right]$, with $M_{0}(x)=x+\alpha$, is a fixed point of $\mathcal{R}$, and it clearly has rotation number $\alpha$. Notice that the "exponents" in (1.15) are precisely the matrix elements of $T$. This suggests of course a generalization to problems with more than one frequency.

This renormalization operator $\mathcal{R}$ (for any $k$ ) has been studied in great detail [129]. To give a very simple application, it can be shown e.g. that if $F$ is a small perturbation of $F_{0}$ with rotation number $\alpha$, then $\mathcal{R}^{n}(F) \rightarrow F_{0}$ as $n \rightarrow \infty$. This in turn can be used to establish a conjugacy between $F$ and $F_{0}$.

The analogous operator can be defined also for other types of maps. Such an operator was studies in connection with the breakup of invariant circles in area-preserving maps of the plane $[68,100,52,101,117]$.

\subsection{Breakup of invariant tori}

Let now $d=2$ and $\omega=\left[\begin{array}{c}\vartheta^{-1} \\ 1\end{array}\right]$, with $\vartheta$ the golden mean $\frac{1}{2} \sqrt{5}+\frac{1}{2}$. By the KAM theorem, a Hamiltonian $H$ close to an integrable Hamiltonian like (1.9) has a smooth invariant torus $\Gamma$ with winding numbers $\omega_{j} / \omega_{d}$. The proof also shows that near this torus, $H$ is essentially integrable, and so the motion is highly ordered and stable. (The same is true in higher dimensions.) In the case $d=2$, an invariant 2-torus has an even stronger stabilizing effect, as it divides the (3 dimensional) energy surface containing it into disjoint invariant regions.

Consider a one-parameter family $\beta \mapsto H^{\beta}$ of Hamiltonians on $\mathbb{T}^{2} \times \mathbb{R}^{2}$, such as

$$
H^{\beta}(q, p)=\omega \cdot p+\frac{1}{2} p_{1}^{2}+\beta\left[\cos \left(q_{1}\right)+\cos \left(q_{1}-q_{2}\right)\right],
$$


which is essentially the Hamiltonian used in [47]. For values of $\beta$ close to 0 , this Hamiltonian has a golden invariant torus, that is, a smooth invariant torus with winding number $\vartheta^{-1}$. This torus is observed to persist as $\beta$ is increased, up to some value $\beta=\beta_{\infty}$, where it breaks up. The breakup is also seen to promote chaotic motion in the form of hyperbolic orbits with golden mean rotation number.

Before the critical point $\beta_{\infty}$, the system has "prominent" periodic orbits (symmetric Birkhoff orbits) for each of the rotation numbers $\frac{1}{2}, \frac{2}{3}, \frac{3}{5}, \frac{5}{8}, \ldots$ associated with the continued fraction expansion of $\theta^{-1}$. Past the critical point, these orbit with rotation number $u_{n} / v_{n}$ turn unstable, at parameter values $\beta_{n}$ that converge to $\beta_{\infty}$ in the limit $n \rightarrow \infty$. The convergence is observed to be asymptotically geometric, with

$$
\lim _{n \rightarrow \infty} \frac{\beta_{n+1}-\beta_{n}}{\beta_{n}-\beta_{n-1}}=\delta^{-1}, \quad \delta=1.6279 \ldots
$$

This ration appears to be universal, in the sense that the same values is observed within a large class of one-parameter families.

The "critical" Hamiltonian $H^{\beta_{\infty}}$ appears to have an invariant torus $\Gamma$ that is nonsmooth. Near this torus, the motion for $H^{\beta_{n+1}}$ looks like that of $H^{\beta_{n}}$, modulo a scaling of time (by $\vartheta$ ) and a scaling of space. The observed eigenvalues of the spatial scaling are

$$
\lambda_{\tau}=\vartheta, \quad \lambda_{x}=\mu_{*} / \lambda_{z} \quad \lambda_{y}=\mu_{\infty} / \lambda_{\tau}, \quad \lambda_{z}=-0.32606 \ldots,
$$

with $\mu_{*}=0.23046 \ldots$. Again, these values appear to be universal.

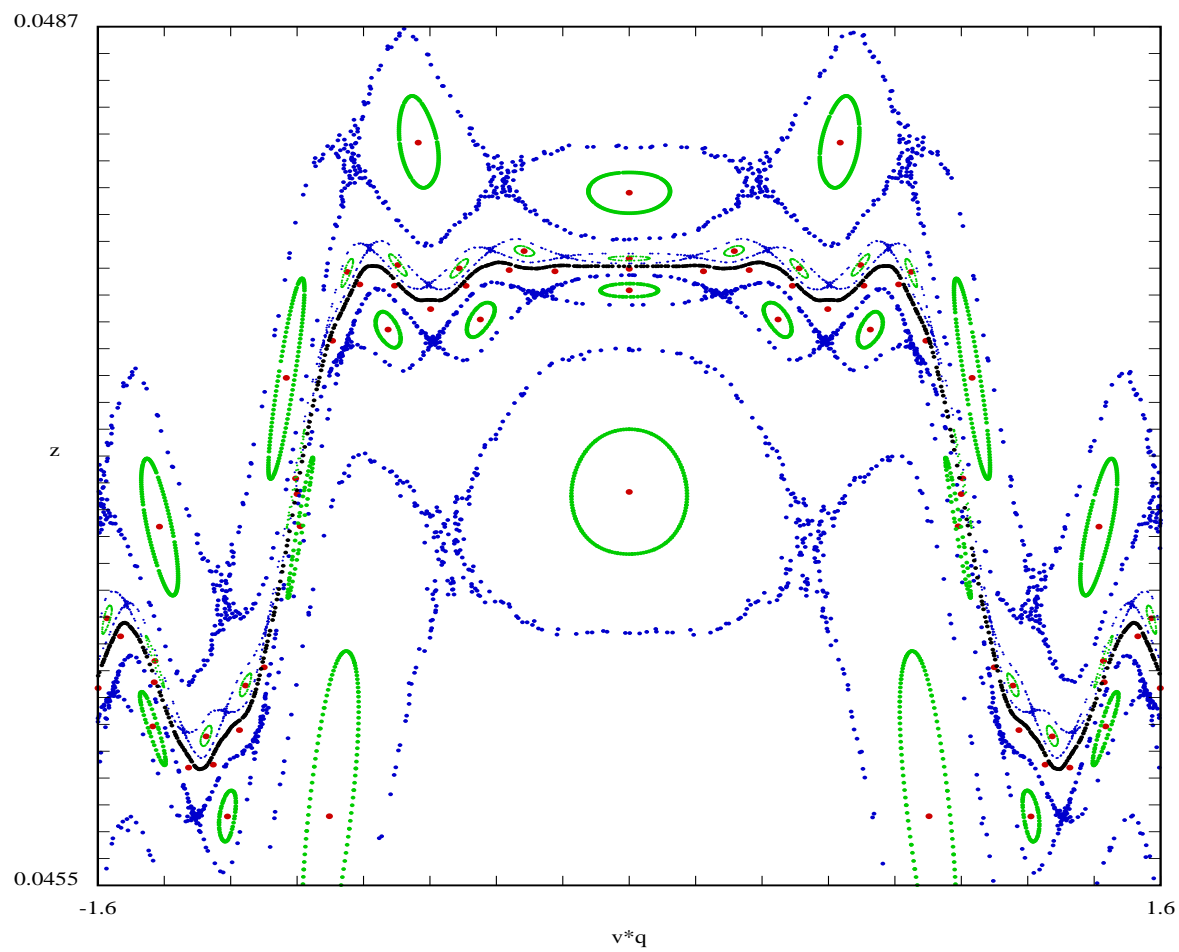

Fig. 2. Orbits for a critical Hamiltonian [2] 
When looking for an explanation, anybody that is familiar with statistical mechanics and phase transitions will immediately try to find an appropriate renormalization group transformation $\mathcal{R}$, acting on a space of Hamiltonians. The expected picture involves the existence of a hyperbolic fixed point $H^{*}$ for $\mathcal{R}$, such that $D \mathcal{R}\left(H^{*}\right)$ has an expanding eigenvalue $\delta=1.6279 \ldots$, and no other spectrum outside the open unit disk.

\section{Renormalization of flows}

After introducing a renormalization group (RG) transformation for Hamiltonian flows, and motivating the choices involved, we generalize the construction to other vector fields on $\mathbb{T}^{d} \times \mathbb{R}^{\ell}$, and to skew flows over $\mathbb{T}^{d}$.

\subsection{Hamiltonian systems}

The first use of renormalization in connection with the breakup of golden invariant tori appears to be by Escande and Doveil [48]. Their transformation contained the following ingredients. One is a frequency scaling $H \mapsto H \circ \mathcal{T}$,

$$
\mathcal{T}(q, p)=\left(T q,\left(T^{*}\right)^{-1} p\right)
$$

where $T^{*}$ denotes the transpose of $T$. Here, $T=\left[\begin{array}{ll}0 & 1 \\ 1 & 1\end{array}\right]$. The remaining ingredients are a scaling of time and momenta, and a canonical change of variables $H \mapsto H \circ U$, designed to obtain a renormalized Hamiltonian of the same form as the original Hamiltonian. The latter was chosen ad-hoc and involved truncations, since only a few selected Fourier modes were being considered.

An alternative approach by Kadanoff, Shenker, and MacKay $[68,100,52,101]$ uses commuting pairs of area-preserving maps. The breakup of invariant circles for these maps produces the same phenomena (and numbers) as described above. After all, one way to obtain an area-preserving map it to start with a Hamiltonian on $\mathbb{T}^{2} \times \mathbb{R}^{2}$, restrict its motion to a surface of constant energy, and then consider the return map to an appropriate plane. Based on the numerical results in [100], there is little doubt that the renormalization picture correctly describes the observed phenomena. The main drawback with this approach is that commuting maps do not constitute a manifold, which makes it hard to discuss some important aspects of renormalization, such as the hyperbolicity of the renormalization operator. (Similar problems are also encountered in one-dimensional dynamics, which lead to the development of alternative approaches, such as the "cylinder renormalization" for Siegel disks $[125,56]$.)

The best rigorous result in this line of work is the existence a nontrivial solution of $\mathcal{R}^{3}(F)=F$, for a renormalization operator $\mathcal{R}$ of the type (1.15), acting on pairs of reversible maps [117]. Whether this solution $F=\left[\begin{array}{l}C \\ M\end{array}\right]$ is a fixed point of $\mathcal{R}$, and whether its components $C$ and $M$ commute, is not known.

In the Hamiltonian approach, it took much longer before accurate computations became possible [2], although some clear improvements to the original scheme were made earlier [107,13]; see also [16] and references therein. The difficulty has to do with the 
choice for the change of variables $H \mapsto H \circ U$. In other problems where renormalization has been applied, the analogue of $U$ can be guessed from the observed scaling. This scaling is typically a contraction on phase space, so it may be reduced to a simple (finite codimension) normal form. But the torus cannot be contracted.

As it turns out [74], it is still possible to find a RG transformation of the form

$$
\mathcal{R}(H)=H \circ \mathcal{T} \quad(\bmod \mathcal{G})
$$

where $\mathcal{G}$ is some "group" of similarity transformations. But it appears that this group needs to be infinite dimensional. Among the possible similarity transformations are

- Scaling of time or energy, $H \mapsto \eta^{-1} H-E$.

- Scaling of momenta $H \mapsto \mu^{-1} H(., \mu \cdot)$

- Change of variables $H \mapsto H \circ U$, with $U$ canonical and homotopic to the identity.

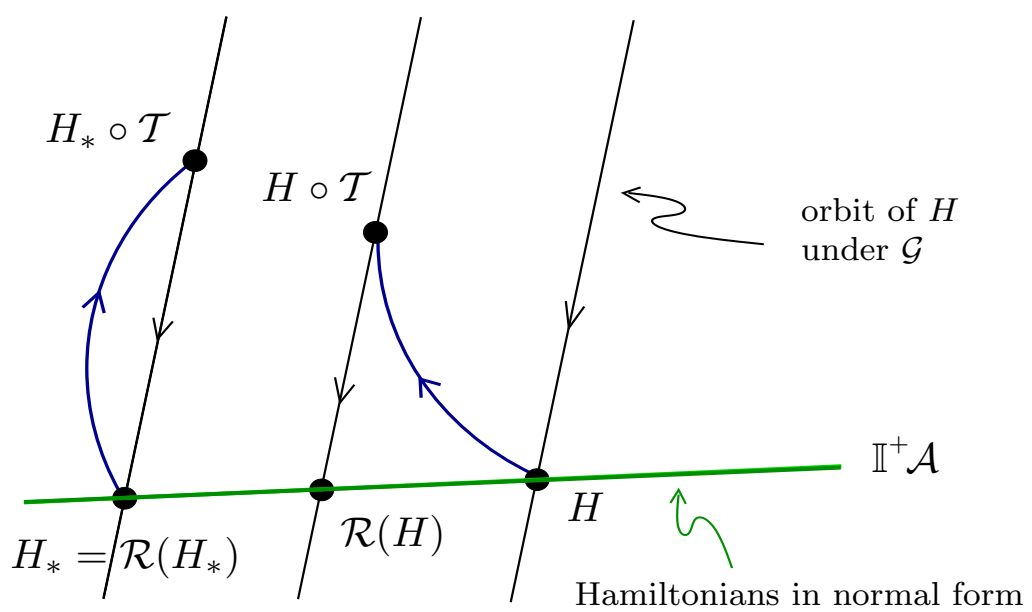

Fig. 3. General form of the RG transformation $\mathcal{R}$

The goal is to find a suitable "normal form" for Hamiltonians, and a map $H \mapsto G_{H} \in \mathcal{G}$, such that $G_{H}(H \circ \mathcal{T})$ is in normal form whenever $H$ is. Using for $G_{H}$ a composition of the three similarity transformations listed above, we have

$$
\mathcal{R}(H)=H^{\prime} \circ \mathcal{U}_{H^{\prime}}, \quad H^{\prime}=\frac{1}{\eta \mu} H \circ \mathcal{T}_{\mu}-E
$$

where $\mathcal{U}_{H^{\prime}}$ is a canonical change of variables,

$$
\mathcal{T}_{\mu}(q, p)=\left(T q, \mu\left(T^{*}\right)^{-1} p\right)
$$

and $E, \eta, \mu$ are normalization constants that may depend on $H$. We will set $E=0$ from now on, and mostly ignore constant terms in Hamiltonians, since such terms do not change 
the vector field. The constants $\eta$ and $\mu$ can be determined e.g. by prescribing the value of two coefficients in the Taylor expansion for the torus average of $\mathcal{R}(H)$. Choosing a suitable normal form that also determines $\mathcal{U}_{H^{\prime}}$ is more delicate. This problem will be discussed in Subsections 2.2 and 2.3.

We expect $\mathcal{R}$ to have at least one integrable fixed point, describing the flow near smooth invariant tori. Such fixed points are in fact easy to find. To be more specific, let $T$ be a matrix in $\operatorname{SL}(d, \mathbb{Z})$ that has two eigenvectors $T \omega=\vartheta_{1} \omega$ and $T \Omega=\vartheta_{2} \Omega$, for two eigenvalues satisfying $\vartheta_{1}>1>\left|\vartheta_{2}\right|$. Consider the integrable Hamiltonians

$$
K(q, p)=f(p)=(\omega \cdot p)+\frac{m}{2}(\Omega \cdot p)^{2},
$$

with $m>0$, unless specified otherwise. Starting with this Hamiltonian $K$, computing $K \circ \mathcal{T}$, and then applying a momentum and energy scaling (including an angle-dependent change of variables would be counter-productive here), we obtain

$$
\begin{aligned}
\mathcal{R}(K)(q, p) & =\eta^{-1} \mu^{-1} f\left(\mu\left(T^{*}\right)^{-1} p\right)-E \\
& =\eta^{-1} \vartheta_{1}^{-1}(\omega \cdot p)+\eta^{-1} \mu \vartheta_{2}^{-2} \frac{m}{2}(\Omega \cdot p)^{2}-E .
\end{aligned}
$$

For $K$ to be a fixed point for $\mathcal{R}$, we need an energy scaling $\eta=\vartheta_{1}^{-1}$, and $E=0$. Ignoring for the time being the case $m=0$, where the momentum scaling $\mu$ is undetermined, we have $\mu=\vartheta_{1}^{-1} \vartheta_{2}^{2}$. Notice that $|\mu|<1$, due to our condition $\vartheta_{1}>1>\left|\vartheta_{2}\right|$, meaning that the momenta $p$ are contracted by the scaling $H \mapsto \mu^{-1} H(., \mu$.$) .$

So far so good. The problem arises when we try to extend $\mathcal{R}$ to Hamiltonians $H$ that depend on the angle variables $q$ as well. Consider e.g. a space $\mathcal{A}_{\rho}$ of Hamiltonians that are analytic in the domain $D_{\rho}$, defined by $\left|\operatorname{Im} q_{j}\right|<\rho$ and $\left|p_{j}\right|<\rho$, with $\rho$ some fixed positive real number. If $H$ is analytic on $D_{\rho}$ then $H^{\prime}=H \circ \mathcal{T}_{\mu}$ is analytic on $\mathcal{T}_{\mu}^{-1} D_{\rho}$. But this new the domain is narrower than $D_{\rho}$ in the angular direction $\omega$, by a factor $\vartheta_{1}^{-1}$. The question is whether this loss of analyticity can be restored by a canonical change of variables $H^{\prime} \mapsto H^{\prime} \circ \mathcal{U}_{H^{\prime}}$. At first, this seems unlikely, since $\mathcal{U}_{H^{\prime}}$ should be close to the identity for $H$ close to $K$, if we want $\mathcal{R}$ to be a smooth map on $\mathcal{A}_{\rho}$. And a fixed domain loss cannot be restored by changes of variables arbitrarily close to the identity. What will save the situation are cancellations.

\subsection{Resonant and nonresonant Hamiltonians}

Motivated by the above, we start by trying to identify the "good" and "bad" terms in the Fourier-Taylor series

$$
H(q, p)=\sum_{(\nu, \alpha) \in I} H_{\nu, \alpha} e^{i \nu \cdot q} p^{\alpha}, \quad p^{\alpha}=\prod_{j} p_{j}^{\alpha_{j}}
$$

where $I=\mathbb{Z}^{d} \times \mathbb{Z}_{+}^{d}$. The Hamiltonian $H$ is analytic on $D_{\rho}$ if and only if the series (2.7) converges on $D_{\rho}$, which is roughly equivalent to

$$
\left|H_{\nu \alpha}\right| \lesssim e^{-\rho|\nu|} \rho^{-|\alpha|}, \quad(\nu, \alpha) \in I \text {. }
$$


Here, $|$.$| denotes the \ell^{1}$ norm. In order to identify which of these conditions are violated for the Hamiltonian $H \circ \mathcal{T}_{\mu}$, consider its Fourier-Taylor series

$$
\left(H \circ \mathcal{T}_{\mu}\right)(q, p)=\sum_{(\nu, \alpha) \in I} H_{\nu, \alpha} e^{i\left(T^{*} \nu\right) \cdot q}\left[\mu\left(T^{*}\right)^{-1} p\right]^{\alpha}
$$

If we consider just the terms with fixed degree $|\alpha|$, then the "bad" terms can be identified as those for which $\left|T^{*} \nu\right|>|\nu|$. A more careful analysis has to take into account that the factor $\mu^{|\alpha|}$ in (2.9) improves convergence in directions where $|\alpha| \rightarrow \infty$.

Alternatively, we can try to identify the "good" modes $e^{i \nu \cdot q} p^{\alpha}$ that do not get expanded under composition with $\mathcal{T}_{\mu}$. Among them are the "resonant" modes, which we now describe. Assume that all eigenvalues of $T$ other than $\vartheta_{1}$ are of modulus $<1$. Then the orthogonal complement of $\omega$ is contracted by $T^{*}$, with respect to some norm on $\mathbb{C}^{d}$ that we will denote by $\|\cdot\|$. Given real numbers $\sigma, \kappa>0$ to be determined later, define

$$
I^{+}=\{(\nu, \alpha) \in I:|\omega \cdot \nu| \leq \sigma\|\nu\| \text { or }|\omega \cdot \nu| \leq \kappa|\alpha|\}, \quad I^{-}=\mathrm{I} \backslash I^{+} .
$$

The "resonant" part $\mathbb{I}^{+} H$ of a Hamiltonian $H$, and its "nonresonant" part $\mathbb{I}^{-} H$, are now defined by restricting the sum in (2.7) to the index set $I^{+}$and $I^{-}$, respectively.

In order to make precise what we mean by non-expanding modes, we need to introduce a norm. Define $\mathcal{A}_{\rho}$ to be the space of Hamiltonians that are analytic on the domain $D_{\rho}$, and continuous on its closure. We equip this space with the norm

$$
\|H\|_{\rho}=\sum_{(\nu, \alpha) \in I}\left|H_{\nu, \alpha}\right| e^{\rho\|\nu\|} \rho^{|\alpha|} .
$$

It is now straightforward to prove the following

Proposition 2.1. If $\sigma,|\mu|$ are positive and sufficiently small, and if $\rho^{\prime}<\rho$ is sufficiently close to $\rho$, then the restriction of $H \mapsto H \circ \mathcal{T}_{\mu}$ to $\mathbb{I}^{+} \mathcal{A}_{\rho^{\prime}}$ is a compact linear operator from $\mathbb{I}^{+} \mathcal{A}_{\rho^{\prime}}$ to $\mathcal{A}_{\rho}$, with operator norm $\leq 1$.

Proof. Pick $(\nu, \alpha)$ in the index set $I^{+}$, and consider the function $E_{\alpha, \nu}(q, p)=e^{i \nu \cdot q} p^{\alpha}$. Then for $r>0$,

$$
\begin{aligned}
\left\|E_{\nu, \alpha} \circ \mathcal{T}_{\mu}\right\|_{\rho} & \leq e^{\rho\left\|T^{*} \nu\right\|}|c \mu \rho|^{|\alpha|} \\
& \leq \exp \left[\rho\left\|T^{*} \nu\right\|-r\|\nu\|+|\alpha| \ln |c \mu \rho / r|\right]\left\|E_{\nu, \alpha}\right\|_{r}
\end{aligned}
$$

where $c$ is some constant depending only on $T$. Consider first $r=\rho^{\prime}$. Clearly, the third term in [...] is always non-positive if $|\mu|>0$ is sufficiently small. If $|\omega \cdot \nu| \leq \sigma|\nu|$ then the sum of the first two terms is also non-positive, provided that $\sigma>0$ has been chosen sufficiently small. This follows from the fact that $\omega^{\perp}$ is contracted by $T^{*}$. Alternatively, if $|\omega \cdot \nu|>\sigma\|\nu\|$ and thus $\|\nu\|<\sigma^{-1} \kappa|\alpha|$, then we can make [..] $\leq 0$ by taking $|\mu|>0$ 
sufficiently small. This shows that the term [...] in (2.12) is non-positive. These arguments clearly extend to $r<\rho$ sufficiently close to $\rho$. Thus, if $H \in \mathbb{I}^{+} \mathcal{A}_{r}$ then

$$
\left\|H \circ \mathcal{T}_{\mu}\right\|_{\rho} \leq \sum_{(\nu, \alpha) \in I^{+}}\left|H_{\nu, \alpha}\right|\left\|E_{\nu, \alpha} \circ \mathcal{T}_{\mu}\right\|_{\rho} \leq \sum_{(\nu, \alpha) \in I^{+}}\left|H_{\nu, \alpha}\right|\left\|E_{\nu, \alpha}\right\|_{r}=\|H\|_{r}
$$

The assertion now follows by taking $r<\rho^{\prime}<\rho$ and using the fact that the inclusion map from $\mathcal{A}_{\rho^{\prime}}$ into $\mathcal{A}_{r}$ is compact.

QED

Notice that the resonant modes, which are essentially the ones that cause small denominator problems in KAM theory, are easy to deal with in this approach.

It should be noted also that the smallness conditions in Proposition 2.1 can easily be replaced by concrete inequalities. To give a concrete example: Using a slightly different definition of $I^{+}$, an analogue of this proposition is proved in [1] for

$$
\left|\vartheta_{2}\right|+\sigma\left(\vartheta_{1}-\left|\vartheta_{2}\right|\right)<\frac{\rho^{\prime}}{\rho}, \quad 0<\left|\frac{\mu}{\vartheta_{d}}\right| e^{\rho \kappa\left(\vartheta_{1}-\left|\vartheta_{2}\right|\right)}<\frac{\rho^{\prime}}{\rho}
$$

\subsection{The change of variables $\mathcal{U}_{H}$}

Proposition 2.1 suggests that we take the resonant Hamiltonians as our "normal form". This requires that the change of variables $\mathcal{U}_{H^{\prime}}$ in equation (2.3) can be chosen in such a way that

$$
\mathbb{I}^{-}\left(H^{\prime} \circ \mathcal{U}_{H^{\prime}}\right)=0
$$

which makes $\mathcal{R}(H)$ again resonant. In other words, the role of $\mathcal{U}_{H^{\prime}}$ would be to eliminate nonresonant modes. Now why should this equation be solvable? Roughly speaking, the reason is that the equation deals mainly with nonresonant functions, which should avoid small denominator problems.

To be more precise, let $K_{0}(q, p)=\omega \cdot p$, and consider a Hamiltonian $H=K_{0}+h$ not too far from $K_{0}$. Denote by $h^{+}$and $h^{-}$the resonant and nonresonant parts of $h$, respectively, and assume that $\varepsilon=\left\|h^{-}\right\|_{\rho}$ is small. If $U$ is a canonical transformation with nonresonant generating function $\phi$ of order $\varepsilon$, then

$$
\begin{aligned}
H \circ U & =H+\{H, \phi\}+\mathcal{O}\left(\varepsilon^{2}\right) \\
& =K_{0}+\left[h-\omega \cdot \nabla_{1} \phi+\{h, \phi\}\right]+\mathcal{O}\left(\varepsilon^{2}\right) .
\end{aligned}
$$

Let us try to solve $\mathbb{I}^{-}(H \circ U)=0$ to first order in $\varepsilon$. The resulting equation for $\phi$ is $\mathbb{I}^{-}[\ldots]=0$, which can be written as

$$
\omega \cdot \nabla_{1} \phi+\mathbb{I}^{-} \widehat{h} \phi=h^{-}
$$

where $\widehat{g}$ denotes the Hamiltonian vector field associated with a Hamiltonian $g$, that is, $\widehat{g} f=\{f, g\}$. The formal solution of this equation is

$$
\left(\omega \cdot \nabla_{1}\right) \phi=\left(\mathbb{I}+\mathbb{L}^{-}\right)^{-1} h^{-},
$$


with

$$
\mathbb{L}^{-}=\mathbb{I}^{-} \widehat{h}\left(\omega \cdot \nabla_{1}\right)^{-1}=\mathbb{I}^{-}\left[\left(\nabla_{2} h\right) \cdot \frac{\nabla_{1}}{\omega \cdot \nabla_{1}}-\left(\nabla_{1} h\right) \cdot \frac{\nabla_{2}}{\omega \cdot \nabla_{1}}\right] .
$$

Here $\mathbb{L}^{-}$is a linear operator on $\mathbb{I}^{-} \mathcal{A}_{\rho}$. Now by the definition of $I^{-}$, the two operators (written as fractions) in square brackets are bounded in norm by $\sigma^{-1}$ and $(\rho \kappa)^{-1}$, respectively. Thus, if $\|\nabla h\|_{\rho}$ is sufficiently small, such that $\left\|\mathbb{L}^{-}\right\|<1$, then equation (2.16) can be solved by a Neumann series. The solution $\psi=\omega \cdot \nabla_{1} \phi$ belongs to $\mathcal{A}_{\rho}$ and is of order $\varepsilon$. The next step would be to solve equation (1.7) for the functions $P$ and $Q$ defining the canonical transformation $U$ generated by $\phi$. Notice that what enters this equation is not $\phi$ directly, but its gradient. This gradient is $\left(\omega \cdot \nabla_{1}\right)^{-1} \nabla \psi$, a function for which we have again convenient bounds.

By construction, the new Hamiltonian $H \circ U$ has a nonresonant part of order $\varepsilon^{2}$. Thus we can solve equation (2.14) by iterating the step $H \mapsto H \circ U$ described above.

A generalization of this procedure will be described in detail in Subsection 7.4.

\section{Remarks.}

- This elimination procedure shrinks domains. However, the domain loss tends to zero with the size of $h^{-}$. Thus, for near-resonant Hamiltonians, the subsequent step $H \mapsto H \circ \mathcal{T}_{\mu}$ more than compensates for this domain loss, making $\mathcal{R}$ analyticity improving.

- It should be stressed that only the nonresonant part of $h$ needs to be small for this procedure to work. The condition $\left\|\mathbb{L}^{-}\right\|<1$ allows for Hamiltonians that are not close to being integrable.

For completeness, let us state a concrete result about the transformation $\mathcal{R}$. Let $\omega=\left(1, \omega_{2}, \ldots, \omega_{d}\right)$ be a fixed vector in $\mathbb{R}^{d}$, whose components span an algebraic number field of degree $d$. We will call such vectors self-similar, for the following reason. It can be shown [74] that there exists a matrix $T \in \mathrm{SL}(d, \mathbb{Z})$ with simple eigenvalues $\vartheta_{j}$ satisfying $\vartheta_{1}>1>\left|\vartheta_{2}\right| \geq \ldots \geq\left|\vartheta_{d}\right|$, such that $T \omega=\vartheta_{1} \omega$. Consider now a fixed matrix $T$ with these properties.

Theorem 2.2. [1] Let $0<\rho<\sigma / \kappa$. If $\rho^{\prime}<\rho$ is sufficiently close to $\rho$ and $\mu \in \mathbb{C}$ satisfies (2.13), then there exists an open neighborhood $B \in \mathcal{A}_{\rho^{\prime}}$ of $K_{0}$ such that $\mathcal{R}: B \rightarrow \mathcal{A}_{\rho}$ is well defined, analytic, and compact.

The version of this theorem given in [1] contains additional information about the domain $B$.

\subsection{Other vector fields}

Flows $\dot{q}=X(q)$ on the torus $\mathbb{T}^{d}$ are a special case of the above, as can be seen by restricting the flow for a Hamiltonian $H=p \cdot X(q)$ to the invariant torus $p=0$. For flows that are not described by a generating function, we have to renormalize the vector field directly.

Let $X$ be a vector field on a manifold $\mathcal{M}$. Consider a change of coordinates $x=\mathcal{U}(y)$ on $\mathcal{M}$. Then $\dot{x}=D \mathcal{U}(y) \dot{y}$. So the pullback of $X$ under $\mathcal{U}$ is

$$
\mathcal{U}^{*} X=(D \mathcal{U})^{-1}(X \circ \mathcal{U})
$$


Consider now $\mathcal{M}=\mathbb{T}^{d} \times \mathbb{R}^{\ell}$, and vector fields near $Z=(\omega, 0)$. In the Hamiltonian case described earlier, we used a the phase space scaling

$$
\mathcal{T}(q, p)=(T q, \mu S p)
$$

with $S$ the transposed inverse of $T$. The same type of scaling is appropriate for other types of vector fields as well, e.g. with $S=$ I whenever $\ell \neq d$. For the pullback of $X=\left(X^{\prime}, X^{\prime \prime}\right)$ under $\mathcal{T}$ we have

$$
\mathcal{T}^{*} X=\left(T^{-1} X^{\prime} \circ \mathcal{T}, \mu^{-1} S^{-1} X^{\prime \prime} \circ \mathcal{T}\right)
$$

The analogue of the renormalization group ( $\mathrm{RG}$ ) transformation (2.3) is now given by

$$
\mathcal{R}(X)=\eta^{-1} \mathcal{U}_{X}^{*} \mathcal{T}^{*} X
$$

where $\mathcal{U}_{X}$ is a change of coordinates that eliminates nonresonant modes. Here, $\eta$ and $\mu$ are normalization constants that may depend on $X$.

This type of RG transformations has been used e.g. in [94,70,79] to linearize torus flows and skew systems, and in [78] to construct invariant tori for general flows near $Z=(\omega, 0)$. When dealing with specific classes of flows, it is useful to choose the projections $\mathbb{I}^{ \pm}$and the change of variables $\mathcal{U}_{X}$ in such a way that the given class is preserved under renormalization. The choice given in [78] does this simultaneously for the following four classes, in the case where $G(q, p)=(-q, p)$. A detailed description of the corresponding elimination procedure $X \mapsto \mathcal{U}_{X}^{*} X$ will be given in Subsection 3.4.

(1) Symmetric: Given a diffeomorphism $G$ of $\mathcal{M}$, a vector field $X$ on $\mathcal{M}$ is symmetric with respect to $G$ if $G^{*} X=X$. The flow for $X$ commutes with $G$. The changes of variables $\mathcal{U}_{X}$ are generated by symmetric vector fields as well.

(2) Reversible: If $G \circ G=\mathrm{I}$, then $X$ is time-reversible with respect to $G$ if $G^{*} X=-X$. The flow for $X$ satisfies $G \circ \Phi_{X}^{t} \circ G=\Phi_{X}^{-t}$. Reversibility is preserved if $\mathcal{U}_{X}$ is generated by symmetric vector fields.

(3) Divergence free: Here, $\operatorname{tr}(D X)=0$. The flow for $X$ is volume preserving. $\mathcal{U}_{X}$ is generated by divergence free vector fields.

(4) Hamiltonian: This case was discussed earlier. Hamiltonian vector fields are also divergence free. Some are time reversible as well, e.g., if $H(q, p)=H(q,-p)$ then $X=\mathbb{J} \nabla H$ is reversible with respect to $G:(q, p) \mapsto(-q, p)$.

For specific results we refer to [78].

\subsection{Skew systems}

Here we discuss in more detail a class of flows called skew flows. These are systems of ODEs with quasiperiodic coefficients. To be more specific, let $\mathfrak{G}$ be a Lie subgroup of $\operatorname{GL}(n, \mathbb{C})$ or $\operatorname{GL}(n, \mathbb{R})$, and let $\mathfrak{A}$ to be the corresponding Lie algebra. Then one considers equations of the type

$$
\dot{y}(t)=F(t) y(t), \quad y(0)=y_{0},
$$

with $y(t) \in \mathfrak{G}$, where $F: \mathbb{R} \rightarrow \mathfrak{A}$ is a quasiperiodic function with $d$ rationally independent frequencies $\omega_{1}, \ldots, \omega_{d}$. Such systems are encountered e.g. in the study of the 
one-dimensional Schrödinger equation with quasiperiodic potentials, where $\mathfrak{G}=\operatorname{SL}(2, \mathbb{R})$. The discrete analogue are products of matrices $F_{i}$ depending quasiperiodically on the index $i$. One of the problem is to find the spectrum of products $F_{m} \cdots F_{2} F_{1}$ in the limit of large $m$. This is trivial in the case where $i \mapsto F_{i}$ is periodic.

We can rewrite $(2.21)$ as

$$
\dot{y}(t)=f\left(q_{0}+t \omega\right) y(t), \quad y(0)=y_{0},
$$

with $f$ a function on $\mathbb{T}^{d}$, taking values in $\mathfrak{A}$. This equation, together with $\dot{q}=\omega$ defines the a vector field $X$ on the manifold $\mathcal{M}=\mathbb{T}^{d} \times \mathfrak{G}$,

$$
X(q, y)=(\omega, f(q) y), \quad f(q) \in \mathfrak{A}, \quad(q, y) \in \mathcal{M}
$$

The flow for $X$ is given by

$$
\Phi_{X}^{t}\left(q_{0}, y_{0}\right)=\left(q_{0}+t \omega, \Psi_{X}^{t}\left(q_{0}\right) y_{0}\right), \quad\left(q_{0}, y_{0}\right) \in \mathcal{M}, \quad t \in \mathbb{R} .
$$

where $t \mapsto \Psi_{X}^{t}\left(q_{0}\right)$ denotes the solution of (2.22) for $y_{0} \in \mathfrak{G}$ the identity.

Classical Floquet theory shows that if $t \mapsto q(t)$ is periodic, and in particular if $d=1$, then the system is reducible. To be more precise, the vector field (2.23) is said to be reducible if there exists a function $U: \mathbb{T}^{d} \rightarrow \mathfrak{G}$, such that

$$
\Psi_{X}^{t}(q)=U(q+t \omega) e^{t C} U(q)^{-1}, \quad t \in \mathbb{R}, \quad q \in \mathbb{T}^{d}
$$

for some constant matrix $C \in \mathfrak{A}$. If $\omega \in \mathbb{R}^{d}$ is fixed, we will also refer to $f$ as being reducible. For another characterization of reducibility, considering the map $\mathcal{U}: \mathcal{M} \rightarrow \mathcal{M}$, defined by

$$
\mathcal{U}(q, y)=(q, U(q) y) .
$$

The pullback of $X=(\omega, f$.$) under this map is given by the equation$

$$
\left(\mathcal{U}^{*} X\right)(q, y)=\left(\omega,\left(\mathcal{U}^{\star} f\right)(q) y\right), \quad \mathcal{U}^{\star} f=U^{-1}\left(f-D_{\omega}\right) U
$$

where $D_{\omega}=\omega \cdot \nabla$. Modulo smoothness assumptions, (2.25) is equivalent to $f=\mathcal{U}^{*} C$.

In the quasiperiodic case, solving $\mathcal{V}_{\star} f \equiv C$ leads to small divisor problems, as in classical KAM theory. Results based on KAM type methods have been obtained in the case where $\mathfrak{G}=\mathrm{SL}(2, \mathbb{R})[34,111,40]$, and for compact Lie groups [84,85]. Another approach to the reducibility problem involves renormalization methods. For discrete time cocycles over rotations by an irrational angle $\alpha$, and for $\mathfrak{G}=\mathrm{SU}(2)$, Rychlik introduced in [115] a renormalization scheme based on a rescaling of first return maps, using the continued fractions expansion of $\alpha$. Improvements of this scheme and global (non-perturbative) results can be found in $[86,87,6]$. In the context of flows, renormalization techniques were used in [97] to prove a local normal form theorem for analytic skew systems with a Brjuno base flow.

Extensions of such RG techniques to skew systems with higher dimensional base maps or flows have become possible with the introduction in [70] of a suitable multidimensional 
continued fractions algorithm. A renormalization scheme based on this algorithm was introduced recently in [79]. It applies to Diophantine skew flows on $\mathbb{T}^{d} \times \mathfrak{G}$, for arbitrary subgroups of $\mathrm{GL}(n, \mathbb{C})$ or $\mathrm{GL}(n, \mathbb{R})$, and for arbitrary dimensions $d, n$. In addition, the smoothness requirements are lowered, from analyticity to a finite degree of differentiability (depending on $d$ and on the Diophantine exponent). The RG transformations themselves are restricted to vector fields $X=(\omega, f$.) with $f$ small. Among the general results are the existence of a stable codimension $d$ manifold (near $f=0$ ) of reducible skew systems. Other near-constant vector fields are mapped to this case by increasing the dimension of the torus. In the case $d=2$ and $\mathfrak{G}=\mathrm{SL}(2, \mathbb{R})$, the stable manifold is identified with the set of skew systems having a fixed fibered rotation number (see Subsection 8.4).

The precise results, and the techniques used to prove them, will be described (below and) in Section 8.

\section{A single renormalization group step}

This section covers the more technical aspects of renormalization. For skew flows, this includes explicit estimates of the type needed (later) to deal with general Diophantine rotation vectors. And for vector fields on $\mathbb{T}^{d} \times \mathbb{R}^{\ell}$, we give a complete description of the main renormalization step: the elimination of nonresonant modes.

\subsection{Skew systems: definitions}

Skew flows are ideal for the description of a complete $R G$ step, since the analysis is quite simple: estimating the action of $\mathcal{R}$ on resonant frequencies takes a few lines, eliminating nonresonant frequencies involves little more than the implicit function theorem, and combining the two is straightforward.

Consider skew flows $X=(\omega, f)$ with $f$ close to zero. Given $\gamma \geq 0$, define $\mathcal{F}_{\gamma}$ to be the Banach space of integrable functions $f: \mathbb{T}^{d} \rightarrow \operatorname{GL}(n, \mathbb{C})$, for which the norm

$$
\|f\|_{\gamma}=\left\|f_{0}\right\|+\sum_{0 \neq \nu \in \mathbb{Z}^{d}}\left\|f_{\nu}\right\|(2\|\nu\|)^{\gamma}
$$

is finite. Here, $f_{\nu}$ denotes the $\nu$-th Fourier coefficient of $f$. Notice that $\mathcal{F}_{\gamma}$ is roughly $C^{\gamma}$. The set of functions in $\mathcal{F}_{\gamma}$ that take values in $\mathfrak{G}$ or $\mathfrak{A}$ will be denoted by $\mathcal{G}_{\gamma}$ or $\mathcal{A}_{\gamma}$, respectively. We will now drop the subscript $\gamma$ if no confusion can arise.

A single RG step for skew flows is associated with a unit vector $\omega \in \mathbb{R}^{d}$, and a matrix $T$ in $\operatorname{SL}(d, \mathbb{Z})$, which we assume now to be given. Let

$$
\mathcal{T}(q, y)=(T(q), y)
$$

The pullback of $X$ under $\mathcal{T}$ is given by

$$
\left(\mathcal{T}^{*} X\right)(q, y)=\left(T^{-1} \omega,\left(\mathcal{T}^{\star} f\right)(q) y\right), \quad \mathcal{T}^{\star} f=f \circ T .
$$

Denote by $\mathcal{K}(r)$ the set of vectors in $\mathbb{R}^{n}$ that are contracted by a factor $\leq r$ by the matrix $T^{*}$. Choose $0<\sigma<\tau<1$, if possible, such that

$$
2 \sigma\|T\|<\tau, \quad \omega^{\perp} \subset \mathcal{K}(\tau / 2) .
$$


The "resonant" part $\mathbb{I}^{+} f$ of a function $f \in \mathcal{F}$, and its "nonresonant" part $\mathbb{I}^{-} f$, are now defined by the equation

$$
\mathbb{I}^{ \pm} f(q)=\sum_{\nu \in I^{ \pm}} f_{\nu} e^{i \nu \cdot q}
$$

where $I^{+}$is the set of integer points in $\mathcal{K}(\tau)$ and $I^{-}$its complement in $\mathbb{Z}^{d}$. We will show below that it is possible to find $\mathcal{U}_{f} \in \mathcal{F}$ close to the identity, such that

$$
\mathbb{I}^{-} \mathcal{U}_{f}^{\star} f=0,
$$

and such that $\mathcal{U}_{f} \in \mathcal{G}$ whenever $f \in \mathcal{A}$. The renormalized function $\mathcal{N}(f)$ and the renormalized vector field $\mathcal{R}(X)$ are now defined by the equation

$$
\mathcal{N}(f)=\eta^{-1} \mathcal{T}^{\star} \mathcal{U}_{f}^{\star} f, \quad \mathcal{R}(X)=\eta^{-1} \mathcal{T}^{*} \mathcal{U}_{f}^{*} X,
$$

where $\eta$ is the norm of $T^{-1} \omega$, so that the torus component of $\mathcal{R}(X)$ is again a unit vector.

\subsection{Skew systems: estimates}

The resonant part of $f$ is easy to deal with:

Lemma 3.1. If $f \in \mathcal{F}$ satisfies $\mathbb{I}^{-} f=\mathbb{E} f=0$, then $\left\|\mathcal{T}^{\star} f\right\| \leq \tau^{\gamma}\|f\|$.

Here, $\mathbb{E} f$ denotes the torus-average of $f$. The proof is one line:

$$
\left\|\mathcal{T}^{\star} f\right\|=\sum_{0 \neq \nu \in I^{+}}\left\|f_{\nu}\right\|(2\|S \nu\|)^{\gamma} \leq \sum_{0 \neq \nu \in I^{+}}\left\|f_{\nu}\right\|(2 \tau\|\nu\|)^{\gamma}=\tau^{\gamma}\|f\| .
$$

Notice the dependence of the contraction factor $\tau^{\gamma}$ on the degree of smoothness $\gamma$.

Next, before we can solve (3.6), we need to estimate some simple linear operators. Given any $n \times n$ matrix $C$, define $\widehat{C} f=f C-C f$ for every function $f \in \mathcal{F}$.

Proposition 3.2. Assume that $\|C\| \leq \sigma / 4$. Then the linear operators $D_{\omega}=\omega \cdot \nabla$ and $\mathcal{D}=D_{\omega}+\widehat{C}$ commute with $\mathbb{I}^{-}$, have bounded inverses when restricted to $\mathbb{I}^{-} \mathcal{F}$, and satisfy

$$
\left\|D_{\omega}^{-1} \mathbb{I}^{-}\right\| \leq \sigma^{-1}, \quad\left\|D_{\omega} \mathcal{D}^{-1} \mathbb{I}^{-}\right\| \leq 2 .
$$

Proof. Clearly, $D_{\omega}, \widehat{C}$, and $\mathbb{I}^{-}$commute with each other. The first inequality in (3.9) follows directly from the fact that $|\omega \cdot \nu|>\sigma$ whenever $\nu$ belongs to $I^{-}$, which is straightforward to check. It implies $\left\|D_{\omega}^{-1} \widehat{C} \mathbb{I}^{-}\right\| \leq 2 \sigma^{-1}\|C\| \leq 1 / 2$, and the indicated bound on $D_{\omega} \mathcal{D}^{-1} \mathbb{I}^{-}=\left(\mathbb{I}+D_{\omega}^{-1} \widehat{C}\right)^{-1} \mathbb{I}^{-}$is now obtained via Neumann series.

QED

Let us recall at this point some facts about

analytic maps. Let $\mathcal{X}$ and $\mathcal{Y}$ be Banach spaces over $\mathbb{C}$, and let $B \subset \mathcal{X}$ be open. We say that $G: B \rightarrow \mathcal{Y}$ is analytic if it is Fréchet differentiable. Thus, sums, products, and compositions of analytic maps are analytic. Equivalently, $G$ is analytic if it is locally bounded, 
and if for all continuous linear maps $f: \mathbb{C} \rightarrow \mathcal{X}$ and $h: \mathcal{Y} \rightarrow \mathbb{C}$, the function $h \circ G \circ f$ is analytic. This shows e.g. that uniform limits of analytic functions are analytic. Assuming that $B$ is a ball of radius $r$ and that $F$ is bounded on $B$, a third equivalent condition is that $G$ has derivatives of all orders at the center of $B$, and that the corresponding Taylor series has a radius of convergence at least $r$ and agrees with $G$ on $B$. See e.g. [62] for more details.

Our next goal is to solve (3.6). Given $f=C+h$ in $\mathcal{F}$ with $C$ constant, we seek a solution of the form $U=\exp \left(\mathcal{D}^{-1} u\right)$, where $u$ is a function in $\mathbb{I}^{-} \mathcal{F}$. We have

$$
\begin{aligned}
\mathbb{I}^{-} \mathcal{U}^{*} f & =\mathbb{I}^{-}\left[e^{-\mathcal{D}^{-1} u}\left(f-D_{\omega}\right) e^{\mathcal{D}^{-1} u}\right] \\
& =\mathbb{I}^{-}\left[\left(\mathrm{I}-\mathcal{D}^{-1} u\right)\left(C+h-D_{\omega}\right)\left(\mathrm{I}+\mathcal{D}^{-1} u\right)\right]+\mathcal{O}(\|h\|\|u\|)+\mathcal{O}\left(\|h\|^{2}\right) \\
& =\mathbb{I}^{-}\left[h-\widehat{C} \mathcal{D}^{-1} u-D_{\omega} \mathcal{D}^{-1}\right]+\mathcal{O}(\|h\|\|u\|)+\mathcal{O}\left(\|u\|^{2}\right) \\
& =h-u+\mathcal{O}(\|h\|\|u\|)+\mathcal{O}\left(\|u\|^{2}\right)
\end{aligned}
$$

So if $\|h\|$ is sufficiently small, then by the implicit function theorem, the equation $\mathbb{I} \mathcal{U}^{*} f=0$ has a solution $u_{f}=h+\mathcal{O}\left(\|h\|^{2}\right)$, and this solution depends analytically on $f$.

Wit a bit more work, one gets an explicit bound on $u_{f}$ for $\|C\| \leq \sigma / 6$ and $\|h\| \leq 2^{-9} \sigma$, and verifies that

$$
\left\|\mathcal{U}_{f}^{*} f-\mathbb{I}^{+} f\right\| \leq 2^{4} \sigma^{-1}\|h\|^{2} .
$$

As a result, we have

Theorem 3.3. [79] Assume that $\sigma$ and $\tau$ satisfy (3.4). Let $f=C+h$, with $C$ constant and $\mathbb{E} h=0$. If $\|C\|<\sigma / 6$ and $\|h\|<2^{-9} \sigma$, then

$$
\mathcal{N}(f)=\eta^{-1}[C+\tilde{h}], \quad\|\tilde{h}\| \leq \frac{3}{2} \tau^{\gamma}\|h\|, \quad|\mathbb{E} \tilde{h}| \leq 2^{4} \sigma^{-1} \tau^{\gamma}\|h\|^{2} .
$$

$\mathcal{N}$ is analytic on the region determined by the given bounds on $C$ and $h$.

Proof. The function $\tilde{h}$ in equation (3.12) is given by $\tilde{h}=\mathcal{T}^{\star}\left[\mathbb{I}^{+} h+\left(\mathcal{U}_{f}^{\star} f-\mathbb{I}^{+} f\right)\right]$. Now we can use Lemma 3.1 and the bound (3.11). In particular, we have

$$
\|\tilde{h}\| \leq \tau^{\gamma}\left(\|h\|+2^{4} \sigma^{-1}\|h\|^{2}\right) \leq \frac{3}{2} \tau^{\gamma}\|h\|
$$

as claimed. The analyticity of $\mathcal{N}$ follows from the analyticity of the map $f \mapsto u_{f}$, the uniform convergence of the exponentials in (3.10), and the chain rule.

QED

Notice that, by construction, if $f$ belongs to $\mathcal{A}$ then so does $\mathcal{N}(f)$. Similarly, if $f$ is real-valued, then so is $\mathcal{N}(f)$.

Since this theorem will be used later with $\|T\|$ very large, requiring $\sigma$ to be very small by (3.4), it should also be noted that the domain of $\mathcal{N}$ is roughly of size $\sigma$, which is due to the factor $\sigma^{-1}$ in the estimate (3.9). 


\subsection{More general vector fields}

We start with some basic estimates for analytic vector fields on $\mathcal{M}=\mathbb{T}^{d} \times \mathbb{R}^{\ell}$, near $Z=(\omega, 0)$. Recall that the $\mathrm{RG}$ transformation for such vector fields is given by

$$
\mathcal{R}(X)=\eta^{-1} \mathcal{U}_{X}^{*} \mathcal{T}_{\mu}^{*} X,
$$

where $\mathcal{U}_{x}$ is a change of coordinates that eliminates nonresonant modes. The definition of resonant and nonresonant modes is analogous to the one used for Hamiltonians, and the proof that $X \mapsto \mathcal{T}_{\mu}^{*} X$ is analyticity improving (and thus compact) when restricted to the resonant subspace is essentially the same as in the Hamiltonian case. Thus, we will describe here only the elimination procedure $X \mapsto \mathcal{U}_{X}^{*} X$. But we will do this in full detail, since the elimination of nonresonant modes is probably the most crucial part of $\mathcal{R}$. The version presented here is taken from [78].

We start with some estimates on the flow generated by an analytic vector field $Y$. On the spaces $\mathbb{C}^{m}$ we use the $\ell^{\infty}$ norm, and for linear operators we use the operator norm.

Given $\rho>0$, denote by $D_{\rho}$ the set of all vectors $(q, p)$ in $\mathbb{C}^{d} \times \mathbb{C}^{\ell}$ characterized by $\|\operatorname{Im} q\|<\rho$ and $\|p\|<\rho$. If $V$ is any complex Banach space, an analytic function $f: D_{\rho} \rightarrow V$ that is $2 \pi$-periodic in each of the variables $q_{j}$ can be written as

$$
f(q, p)=\sum_{(\nu, \alpha) \in I} f_{\nu, \alpha} e^{i \nu \cdot q} p^{\alpha}, \quad \nu \cdot q=\sum_{j} \nu_{j} q_{j} \quad p^{\alpha}=\prod_{j} p_{j}^{\alpha_{j}}
$$

where $I=\mathbb{Z}^{d} \times \mathbb{N}^{\ell}$. Define $\mathcal{A}_{\rho}(V)$ to be the space of all functions (3.15) for which the norm

$$
\|f\|_{\rho}=\sum_{(\nu, \alpha) \in I}\left\|f_{\nu, \alpha}\right\| e^{\rho|\nu|} \rho^{|\alpha|}
$$

is finite. Here, $|\nu|=\sum_{j}\left|\nu_{j}\right|$, and $|\alpha|$ is defined analogously. If no ambiguity can arise, we will simply write $\mathcal{A}_{\rho}$ in place of $\mathcal{A}_{\rho}(V)$. The operator norm of a continuous linear map $L$ on $\mathcal{A}_{\rho}$ will be denoted by $\|L\|_{\rho}$.

It is easy to check that if $V$ is a Banach algebra, then so is $\mathcal{A}_{\rho}(V)$. Another basic fact about the spaces $\mathcal{A}_{\rho}$ is the following. Let $\pi_{1}(q, p)=(q, 0)$.

Proposition 3.4. Let $0 \leq \rho^{\prime} \leq \rho$. Let $X \in \mathcal{A}_{\rho}(V)$ and $Y=\left(Y^{\prime}, Y^{\prime \prime}\right)$, with $Y^{\prime} \in \mathcal{A}_{\rho^{\prime}}\left(\mathbb{C}^{d}\right)$ and $Y^{\prime \prime} \in \mathcal{A}_{\rho^{\prime}}\left(\mathbb{C}^{\ell}\right)$. Then

(a) $(D X) Y \in \mathcal{A}_{\rho^{\prime}}(V)$ and $\|(D X) Y\|_{\rho^{\prime}} \leq\left(\rho-\rho^{\prime}\right)^{-1}\|X\|_{\rho}\|Y\|_{\rho^{\prime}}$, if $\rho^{\prime}<\rho$.

(b) $X \circ\left(\pi_{1}+Y\right) \in \mathcal{A}_{\rho^{\prime}}(V)$ and $\left\|X \circ\left(\pi_{1}+Y\right)\right\|_{\rho^{\prime}} \leq\|X\|_{\rho}$, if $\rho^{\prime}+\left\|Y^{\prime}\right\|_{\rho^{\prime}},\left\|Y^{\prime \prime}\right\|_{\rho^{\prime}} \leq \rho$.

The flow $\Phi_{Y}$ associated with a vector field $Y$ can be estimated e.g. by comparing it to the flow $\Phi_{Z}$ for a constant real vector field $Z=(\omega, 0)$. The following bound is obtained by applying a standard contraction mapping argument to the equation

$$
G(t)=\int_{0}^{t}\left[(Y-Z) \circ \Phi_{Z}^{s}\right] \circ[\mathrm{I}+G(s)] d s,
$$

satisfied by the difference $G(t)=\Phi_{Y}^{t}-\Phi_{Z}^{t}$. 
Proposition 3.5. Let $\tau$ be a positive real number and $Y$ a vector field in $\mathcal{A}_{\rho}$, such that $\tau\|Y-Z\|_{\rho}<r<\rho$. Then the equation (3.17) has a unique continuous solution $G \in \mathcal{A}_{\rho-r}$ on the interval $|t| \leq \tau$, and

$$
\left\|\Phi_{Y}^{t}-\Phi_{Z}^{t}\right\|_{\rho-r} \leq\|t(Y-Z)\|_{\rho} .
$$

Next, we consider the pushforward for the time $t$ map $\Phi_{Y}^{t}$. If $X$ and $Y$ are arbitrary vector fields, define $\widehat{Y} X=[Y, X]=(D X) Y-(D Y) X$.

Proposition 3.6. Let $0<r<\rho$ and $t \in \mathbb{R}$. Let $Y$ and $X$ be two vector fields in $\mathcal{A}_{\rho}$, satisfying $\|t Y\|_{\rho} \leq r \varepsilon$ and $\|t D Y\|_{\rho} \leq s \varepsilon$, with $\varepsilon \leq 1 / 6$. Then $\left(\Phi_{Y}^{t}\right)^{*} X$ belongs to $\mathcal{A}_{\rho-r}$, and

$$
\begin{aligned}
\left\|\left(\Phi_{Y}^{t}\right)^{*} X-X\right\|_{\rho-r} & \leq 3 e^{s}\|X\|_{\rho} \varepsilon \\
\left\|\left(\Phi_{Y}^{t}\right)^{*} X-X-t[Y, X]\right\|_{\rho-r} & \leq 7 e^{s}\|X\|_{\rho} \varepsilon^{2} .
\end{aligned}
$$

Proof. It suffices to consider $t=1$, since we can rescale $(t, Y)$ to $(1, t Y)$. Let $n$ be a fixed positive integer. By using Proposition 3.4, and Cauchy's formula with contour $|z|=1$, to estimate

$$
(D X) Y=n \varepsilon \frac{d}{d z}\left[X \circ\left(\mathrm{I}+\frac{z}{n \varepsilon} Y\right)\right]_{z=0},
$$

we obtain the bound

$$
\|\widehat{Y} X\|_{\rho^{\prime}-r / n} \leq(n \varepsilon+s \varepsilon)\|X\|_{\rho},
$$

where $\rho^{\prime}=\rho$. This bound can be iterated $n$ times, with $\rho^{\prime}$ decreasing by $r / n$ after each step, and we find

$$
\begin{aligned}
\frac{1}{n !}\left\|(\widehat{Y})^{n} X\right\|_{\rho-r} & \leq \frac{1}{n !}(n+s)^{n} \varepsilon^{n}\|X\|_{\rho} \\
& \leq \frac{n^{n}}{n !} e^{s} \varepsilon^{n}\|X\|_{\rho} \leq \frac{1}{2}(e \varepsilon)^{n} e^{s}\|X\|_{\rho} .
\end{aligned}
$$

In the last inequality, we have used Stirling's formula. Now

$$
\left\|\left(\Phi_{Y}^{1}\right)^{*} X-X\right\|_{\rho-r}=\left\|\sum_{n=1}^{\infty} \frac{1}{n !}(\widehat{Y})^{n} X\right\|_{\rho-r} \leq \frac{\varepsilon}{2} \cdot \frac{e^{s+1}}{1-e \varepsilon}\|X\|_{\rho},
$$

and the first bound in (3.19) follows. The second bound is obtained analogously, with the sum in (3.23) starting at $n=2$.

QED

\subsection{A general elimination procedure}

Denote by $\mathcal{A}_{\rho}^{\prime}$ the space of vector fields on $D_{\rho}$ whose derivatives belong to $\mathcal{A}_{\rho}$. On this space consider the norm

$$
\|Y\|_{\rho}^{\prime}=\|D Y\|_{\rho}+\|Y\|_{\rho} .
$$


Let $\mathbb{I}^{-}$be a fixed but arbitrary projection operator, defined on all the spaces $\mathcal{A}_{\rho}$, and having norm one. We also fix $0<\rho^{\prime}<\rho$. Let $Z$ be any fixed vector field in $\mathcal{A}_{\rho}^{\prime}$ with the property that there exists a positive constant $a \leq 1$ such that

$$
\mathbb{I}^{-} Z=0, \quad\left\|\mathbb{I}^{-}[Y, Z]\right\|_{r} \geq a\|Y\|_{r}^{\prime},
$$

for all $\rho^{\prime} \leq r \leq \rho$, and for all $Y \in \mathbb{I}^{-} \mathcal{A}_{r}^{\prime}$. Notice that for the choices of $Z$ and $\mathbb{I}^{-}$used in Subsection 2.2 (with $\kappa \sim \sigma$ ) and Subsection 3.1, the constant $a$ is roughly equal to $\sigma$.

The goal is to show that if $X$ is sufficiently close to $Z$ in $\mathcal{A}_{\rho}^{\prime}$, then there exists an analytic change of coordinates $\mathcal{U}_{X}: D_{\rho^{\prime}} \rightarrow D_{\rho}$, such that $\mathcal{U}_{X}^{*} X$ belongs to $\mathcal{A}_{\rho}$ and satisfies

$$
\mathbb{I}^{-} \mathcal{U}_{X}^{*} X=0
$$

We start by determining an approximate solution of this equation, given by the time one flow $\Phi_{Y}^{1}$ of a vector field $Y=\mathbb{I}^{-} Y$. To first order in $Y$, the equation (3.26) reduces to

$$
\mathbb{I}^{-}(X+[Y, X])=0
$$

Proposition 3.7. Let $0<r<\rho$. Let $X$ be a vector field in $\mathcal{A}_{\rho}^{\prime}$, satisfying

$$
\|X-Z\|_{\rho}^{\prime} \leq \frac{1}{4} a, \quad\left\|\mathbb{I}^{-} X\right\|_{\rho} \leq \frac{1}{12} a r .
$$

Then the equation (3.27) has a unique solution $Y \in \mathbb{I}^{-} \mathcal{A}_{\rho}$. The vector field $Y$ satisfies $\|Y\|_{\rho} \leq \frac{2}{a}\left\|\mathbb{I}^{-} X\right\|_{\rho}$. Furthermore, $\left(\Phi_{Y}^{1}\right)^{*} X$ belongs to $\mathcal{A}_{\rho-r}$ and satisfies

$$
\begin{aligned}
\left\|\left(\Phi_{Y}^{1}\right)^{*} X-X\right\|_{\rho-r} & \leq \frac{6 e^{r}}{a r}\left\|\mathbb{I}^{-} X\right\|_{\rho}\|X\|_{\rho}, \\
\left\|\left(\Phi_{Y}^{1}\right)^{*} X-X-[Y, X]\right\|_{\rho-r} & \leq \frac{28 e^{r}}{(a r)^{2}}\left\|\mathbb{I}^{-} X\right\|_{\rho}^{2}\|X\|_{\rho} .
\end{aligned}
$$

Proof. The first condition in (3.28) implies that

$$
\|[Y, X-Z]\|_{\rho} \leq 2\|Y\|_{\rho}^{\prime}\|X-Z\|_{\rho}^{\prime} \leq \frac{a}{2}\|Y\|_{\rho}^{\prime}
$$

for every $Y \in \mathcal{A}_{\rho}^{\prime}$. As a consequence, we have

$$
\left\|\mathbb{I}^{-}[Y, X]\right\|_{\rho} \geq\left\|\mathbb{I}^{-}[Y, Z]\right\|_{\rho}-\left\|\mathbb{I}^{-}[Y, X-Z]\right\|_{\rho} \geq \frac{a}{2}\|Y\|_{\rho}^{\prime},
$$

whenever $Y$ belongs to $\mathbb{I}^{-} \mathcal{A}_{\rho}^{\prime}$. This shows that the linear operator $\mathbb{I}^{-} \widehat{X}: \mathbb{I}^{-} \mathcal{A}_{\rho}^{\prime} \rightarrow \mathbb{I}^{-} \mathcal{A}_{\rho}$ has a bounded inverse, and in particular, that the equation (3.27) has a unique solution $Y \in \mathbb{I}^{-} \mathcal{A}_{\rho}^{\prime}$. The bound (3.31) also shows that this solution satisfies

$$
\|Y\|_{\rho} \leq \frac{2}{a}\left\|\mathbb{I}^{-} X\right\|_{\rho}, \quad\|D Y\|_{\rho} \leq \frac{2}{a}\left\|\mathbb{I}^{-} X\right\|_{\rho} .
$$


The remaining claims now follow from Proposition 3.6, setting $\varepsilon=\frac{2}{a r}\left\|\mathbb{I}^{-} X\right\|_{\rho}$ and $s=r$. QED

Our goal is to iterate the map $X \mapsto\left(\Phi_{Y}^{1}\right)^{*} X$ described in Proposition 3.7, by starting with a vector field $X=X_{0}$ and setting

$$
X_{n+1}=\left(\Phi_{Y_{n}}^{1}\right)^{*} X_{n}, \quad \mathbb{I}^{-}\left(X_{n}+\left[Y_{n}, X_{n}\right]\right)=0
$$

for $n=0,1, \ldots$ The expectation is that the maps

$$
U_{n}=\Phi_{Y_{0}}^{1} \circ \Phi_{Y_{1}}^{1} \circ \ldots \circ \Phi_{Y_{n-1}}^{1}
$$

converge to a solution $\mathcal{U}_{X}$ of equation (3.26), as $n$ tends to infinity.

Let now $r=\rho-\rho^{\prime}$. Choose $R \geq\|Z\|_{\rho}+a$ and $\varepsilon \geq 0$, subject to the constraints

$$
\varepsilon \leq 2^{-6} a r, \quad \varepsilon \leq 2^{-9} a^{2} e^{-r}(1+r)^{-1} R^{-1} .
$$

Lemma 3.8. [78] If $X$ is a vector field in $\mathcal{A}_{\rho}^{\prime}$ such that

$$
\|X-Z\|_{\rho}^{\prime} \leq 2^{-3} a, \quad\left\|\mathbb{I}^{-} X\right\|_{\rho} \leq \varepsilon,
$$

with $\varepsilon$ satisfying (3.35), then $U_{n}$ converges in the affine space $\mathrm{I}+\mathcal{A}_{\rho^{\prime}}$ to a function $\mathcal{U}_{X}$ that takes values in $D_{\rho}$. The map $X \mapsto \mathcal{U}_{X}$ is continuous in the region defined by (3.36), analytic in the interior of this region, and satisfies the bounds

$$
\left\|\mathcal{U}_{X}-\mathrm{I}\right\|_{\rho^{\prime}} \leq \frac{3}{a}\left\|\mathbb{I}^{-} X\right\|_{\rho}, \quad\left\|\mathcal{U}_{X}^{*} X-X\right\|_{\rho^{\prime}} \leq 32 R \frac{e^{r}}{a r}\left\|\mathbb{I}^{-} X\right\|_{\rho}
$$

Proof. Let $\rho_{0}=\rho$, and for $m=0,1, \ldots$ define $\rho_{m+1}=\rho_{m}-2 r_{m}$, where $r_{m}=2^{-m-2} r$. Our first goal is to prove that (3.33) defines a sequence of vector fields $X_{m} \in \mathcal{A}_{\rho_{m}}^{\prime}$, satisfying

$$
\left\|X_{m}-X_{m-1}\right\|_{\rho_{m}}^{\prime} \leq 2^{-m-3} a, \quad\left\|\mathbb{I}^{-} X_{m}\right\|_{\rho_{m}} \leq 8^{-m} \varepsilon
$$

If we define $X_{-1}=Z$ and $X_{0}=X$, then these bounds hold for $m=0$ by (3.36). Assume now that (3.38) holds for $m \leq n$. Then, by summing up the bounds on $X_{m}-X_{m-1}$ for $m \leq n$, we obtain the first inequality in

$$
\left\|X_{n}-Z\right\|_{\rho_{n}}^{\prime} \leq \frac{1}{4} a, \quad\left\|\mathbb{I}^{-} X_{n}\right\|_{\rho_{n}} \leq 4^{-n-2} a r_{n}
$$

The second inequality follows from (3.38), by substituting the first bound in (3.35) on $\varepsilon$. Thus, Proposition 3.7 guarantees a unique solution to (3.33), and it yields the bounds

$$
\left\|X_{n+1}-X_{n}\right\|_{\rho_{n}-r_{n}} \leq 6 \frac{e^{r}}{a r} 4^{-n+1} R \varepsilon, \quad\left\|\mathbb{I}^{-} X_{n+1}\right\|_{\rho_{n}-r_{n}} \leq 7 \frac{e^{r}}{(a r)^{2}} 4^{-2 n+3} R \varepsilon^{2}
$$


Here, we have used also that $\left\|X_{n}\right\|_{\rho_{n}} \leq R$, which follows from the first inequality in (3.39). By using the second condition in (3.35), together with the fact that $\|F\|_{\rho_{n}-2 r_{n}}^{\prime} \leq$ $r_{n}^{-1}\|F\|_{\rho_{n}-r_{n}}$, we now obtain (3.38) for $m=n+1$ from the bounds (3.40).

Next, consider the functions $\phi_{k}=\Phi_{Y_{k}}^{1}-$ I. By Proposition 3.5 and Proposition 3.7,

$$
\left\|\phi_{k}\right\|_{\rho_{k-1}} \leq\left\|Y_{k}\right\|_{\rho_{k}} \leq \frac{2}{a}\left\|\mathbb{I}^{-} X_{k}\right\|_{\rho_{k}}<r_{k}
$$

This shows that $U_{m, n}=\Phi_{Y_{m}}^{1} \circ \Phi_{Y_{m+1}}^{1} \circ \ldots \circ \Phi_{Y_{n-1}}^{1}$ defines a function in $\mathrm{I}+\mathcal{A}_{\rho_{n}}$ that takes values in $D_{\rho_{m}}$. Here, and in what follows, it is assumed that $0 \leq m<n$. Setting $U_{k, k}=\mathrm{I}$, we have the bound

$$
\left\|U_{n}-U_{m}\right\|_{\rho^{\prime}}=\left\|\sum_{k=m}^{n-1} \phi_{k} \circ U_{k+1, n}\right\|_{\rho^{\prime}} \leq \sum_{k=m}^{n-1}\left\|\phi_{k}\right\|_{\rho_{k-1}} \leq \sum_{k=m}^{n-1} \frac{2}{a} 8^{-k} \varepsilon .
$$

This shows that $n \mapsto U_{n}$ converges in $\mathrm{I}+\mathcal{A}_{\rho^{\prime}}$ to a limit $\mathcal{U}_{X}$ that takes values in $D_{\rho}$, and that satisfies the first inequality in (3.37) if we set $\varepsilon=\left\|\mathbb{I}^{-} X\right\|_{\rho}$. Clearly, $X_{n} \rightarrow \mathcal{U}_{X} X$ in $\mathcal{A}_{\rho^{\prime}}$. The second inequality in (3.37) is now obtained by using the first bound in (3.40). The analyticity of the map $X \mapsto \mathcal{U}_{X}$ follows from the uniform convergence of $U_{n} \rightarrow \mathcal{U}_{X}$. QED

Under the same assumptions as in Lemma 3.8, is is straightforward to prove the additional bound

$$
\left\|\mathcal{U}_{X}^{*} X-X-[Y, X]\right\|_{\rho^{\prime}} \leq C a^{-3}\left\|\mathbb{I}^{-} X\right\|_{\rho}^{2},
$$

where $Y$ is the vector field described in Proposition 3.7. Here, $C$ is a constant that only depends on $\rho, \rho^{\prime}$, and $\|Z\|_{\rho}$.

\section{A nontrivial RG fixed point}

In this section we sketch the construction [76] of a nontrivial fixed point for $\mathcal{R}$ and describe some numerical results which indicate that there exists an analogous fixed point of $\mathcal{R}^{12}$ for non-twist flows.

\subsection{Observations and result}

As described in Subsection 1.6, numerical experiments with one-parameter families $\beta \mapsto$ $H^{\beta}$ of Hamiltonians on $\mathbb{T}^{2} \times \mathbb{R}^{2}$, whose golden invariant torus breaks up as $\beta$ is increased past some critical value $\beta_{\infty}$, also display sequences of bifurcations that accumulate at the critical point. If $\beta_{n}$ denotes the parameter value where the symmetric Birkhoff orbit with rotation number $u_{n} / u_{n+1}$ becomes unstable, where $u_{n}$ is the $n$-th Fibonacci number, then the observation is that $\left(\beta_{n}-\beta_{n-1}\right) /\left(\beta_{n+1}-\beta_{n}\right)$ converges to a universal number $\delta=1.6279 \ldots$ Furthermore, the orbit structure of the critical Hamiltonian $H^{\beta_{\infty}}$ is selfsimilar near the golden torus, described by the universal constants (1.18).

The standard explanation involves the existence of a nontrivial fixed point $H_{*}$ for a RG transformation $\mathcal{R}$. In this explanation, $\delta$ is the expanding eigenvalue of $D \mathcal{R}\left(H_{*}\right)$. 
The other universal constants like $\mu_{*}$ and $\lambda_{z}$ are related to the re-scaling of $H_{*}$ during renormalization. In particular, $\mu_{*}$ it the value at $H_{*}$ of the scaling $\mu=\mu_{H}$ that appears in the renormalization step $H \mapsto H \circ \mathcal{T}_{\mu}$.

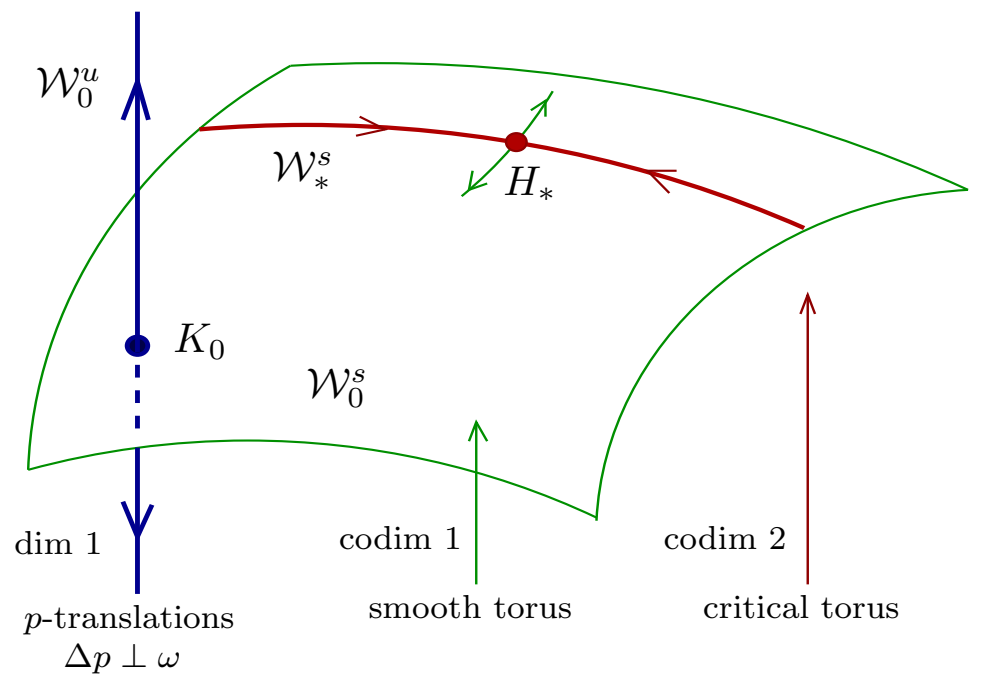

Fig. 4. Expected RG picture for the breakup of invariant tori

When trying to find an RG fixed point such as $H_{*}$, it is useful to determine first possible subclasses of Hamiltonians that are invariant under renormalization. If the phenomena under investigation is observed for a family $\beta \mapsto H^{\beta}$ in such a class, then the intersection point $H^{\beta_{\infty}}$ of this family with the stable manifold at $H_{*}$ belongs to that class, and thus the same should be true for the fixed point $H_{*}$. As was observed already in [74], one such class is the set of all Hamiltonians of the form

$$
H(q, p)=\omega \cdot p+h(q, p), \quad h(q, p)=\sum_{(\nu, k) \in I} h_{\nu, k} \cos (\nu \cdot q)(\Omega \cdot p)^{k}
$$

where $\omega=\left[\begin{array}{c}\vartheta^{-1} \\ 1\end{array}\right]$ and $\Omega=\left[\begin{array}{c}1 \\ -\vartheta^{-1}\end{array}\right]$ are the two eigenvectors of the matrix $T=\left[\begin{array}{ll}0 & 1 \\ 1 & 1\end{array}\right]$, associated with the eigenvalues $\vartheta$ (golden mean) and $-\vartheta^{-1}$. Besides being even in $q$ (time reversibility), these Hamiltonians have the property that the quantity $\omega \cdot q$ evolves linearly in time.

We shall now describe a result $[76,77]$ concerning the existence of the fixed point $H_{*}$. Here, the resonant part $\mathbb{I}^{+} H$ of a Hamiltonian $H$ is defined by restricting the sum in (4.1) to pairs $(\nu, k)$ with the property that $|\omega \cdot \nu| \leq \sigma|\Omega \cdot \nu|$ or $|\omega \cdot \nu|<\kappa k$. Recall that the RG transformation (2.3) involves solving the equation $\mathbb{I}^{-}\left(H \circ \mathcal{U}_{H}\right)=0$. The solution that was described earlier yields $\mathcal{U}_{H}$ as the composition $U_{\phi_{1}} \circ U_{\phi_{2}} \circ \ldots$ of canonical transformation $U_{\phi_{n}}$ close to the identity, with small nonresonant generating functions $\phi_{n}$. Since we are dealing now with Hamiltonians that are far from integrable, we need to include a canonical 
change of variables, say $U_{\phi_{0}}$, that is not close to the identity. The (nonresonant) generating function $\phi_{0}$ for such a transformation was determined in [76], by solving numerically

$$
H_{1}^{\prime} \circ U_{\phi_{0}} \approx H_{1}, \quad \mathbb{I}^{-} H_{1}=0, \quad H_{1}^{\prime}=\eta^{-1} \mu^{-1} H_{1} \circ \mathcal{T}_{\mu},
$$

As a result, the remaining factor $U_{\phi_{1}} \circ U_{\phi_{2}} \circ \ldots$ can be expected to be close to the identity.

The RG transformation used in $[76,77]$ can now be written as

$$
\mathcal{R}=\mathcal{N} \circ \mathcal{L} \circ \mathcal{S}
$$

where

$$
\begin{aligned}
(\mathcal{S} H)(q, p) & =c_{H} H\left(q, p / c_{H}\right), \\
\mathcal{L} H & =\eta_{H}^{-1} \mu_{0}^{-1} H \circ \mathcal{T}_{\mu_{0}} \circ U_{\phi_{0}}, \\
\mathcal{N}(H) & =H \circ \mathcal{U}_{H} .
\end{aligned}
$$

Here, $c_{H}=2 h_{0,2}, \eta_{H}^{-1}=\vartheta$, and $\mathcal{U}_{H}$ is a canonical transformation, determined by solving equation (2.14). Notice that the canonical transformation $U_{\phi_{0}}$ has been combined with other linear parts into a linear operator $\mathcal{L}$, instead of incorporating it into $\mathcal{U}_{H}$.

In Subsection 5.3 we will define a function space $\mathcal{B}_{\rho}$ for function $h$ of the form (4.1), similar to the spaces considered earlier. (Here, we only consider the case $\delta=0$.) The Hamiltonians considered then belong to the affine space $\mathcal{H}_{\rho}=K_{0}+\mathcal{B}_{\rho}$, where $K_{0}(q, p)=$ $\omega \cdot p$. Function in $\mathcal{H}_{\rho}$ that take real values for real arguments will be referred to as real.

Theorem 4.1. [76] There exists an even real resonant Fourier-Taylor polynomial $h_{1}$, an odd real Fourier-Taylor polynomial $\phi_{0}$, a choice of the parameters $\sigma, \kappa, \rho$, and an open neighborhood $B$ of $K_{0}+h_{1}$ in $\mathcal{H}_{\rho}$, such that the following holds. The transformation $\mathcal{R}$ is well defined, analytic, and compact as a map from $B$ to $\mathcal{H}_{\rho}$. It has a unique fixed point $H_{*}$ in $B$, which is real analytic and exhibits a nontrivial scaling, in the sense that $0<\mu\left(H_{*}\right)<\vartheta^{-3}$.

Rigorous bounds on the universal constants can be found in [77].

The strategy for proving Theorem 4.1 will be described below. We note that the Hamiltonians considered here are not necessarily close to integrable. However, if $H^{\prime}=\mathcal{L} H$, with $H$ close to the approximate fixed point $H_{1}=K_{0}+h_{1}$, then $H^{\prime}$ is close to $\mathcal{L} H_{1}$. And by construction, $\mathcal{L} H_{1} \approx H_{1}$. Thus, it suffices to consider $\mathcal{N}$ near the Hamiltonian $H_{1}$, which is resonant.

\subsection{Strategy of proof}

Theorem 4.1 is proved by converting the fixed point problem for $\mathcal{R}$, to a fixed point problem for a Newton-like map $\mathcal{M}$ associated with $\mathcal{R}$,

$$
\mathcal{M}(h)=h+\mathcal{R}\left(H_{1}+M h\right)-\left(H_{1}+M h\right),
$$

where $M$ is an approximate inverse of $\mathbb{I}-D \mathcal{R}\left(H_{1}\right)$. The goal is then to show that $\mathcal{M}$ is a contraction near the approximate fixed point $H_{1}$. This is a common strategy in computerassisted proofs; see e.g. [80] and references therein. It usually involves explicit bounds on 
a finite dimensional truncation of $\mathcal{M}$, and error bounds for the terms that were truncated. In the case at hand, however, working with a truncation of the full map $\mathcal{M}$ turned out to be computationally prohibitive. This problem is solved by using the fact that $\mathcal{M}$ only needs to be estimated on a small open set. More specifically, we approximate the most complex part of $\mathcal{M}$, which is the map $\mathcal{N}$, by a much simpler affine map $\mathcal{N}_{1}$, and then estimate the difference between the two.

The map $\mathcal{N}_{1}$ is defined by the equation

$$
\mathcal{N}_{1}\left(H_{1}+f_{1}\right)=H_{1}+\mathbb{I}^{+}\left(f_{1}+\left\{H_{1}, \phi_{1}\right\}\right),
$$

where $\phi_{1}$ is the solution of $\mathbb{I}^{-}\left(f_{1}+\left\{H_{1}, \phi_{1}\right\}\right)=0$. Then $\mathcal{N}_{1}$ is an approximation of $\mathcal{N}$, in the sense that $\mathcal{N}\left(H_{1}+f_{1}\right)-\mathcal{N}_{1}\left(H_{1}+f_{1}\right)$ vanishes to first order in $f_{1}$. It contains all but the first of the elimination steps described in the last subsection. The map $\mathcal{N}_{1}$ can now be used to define an approximate RG transformation

$$
\mathcal{R}_{1}=\mathcal{N}_{1} \circ \mathcal{L} \circ \mathcal{S}
$$

Notice that $\mathcal{R}_{1}$ is nonlinear, but only due to the trivial scaling transformation $\mathcal{S}$. Define

$$
\begin{aligned}
& \widetilde{\mathcal{S}}\left(H_{1}, f\right)=\mathcal{S}\left(H_{1}+f\right)-\mathcal{S}\left(H_{1}\right)-D \mathcal{S}\left(H_{1}\right) f \\
& \widetilde{\mathcal{R}}\left(H_{1}, f\right)=D \mathcal{N}_{1}\left(H_{1}\right) \mathcal{L} \widetilde{\mathcal{S}}\left(H_{1}, f\right) .
\end{aligned}
$$

Then the map $\mathcal{M}$ can be rewritten as

$$
\begin{aligned}
\mathcal{M}(h)= & \left(\mathcal{R}_{1}\left(H_{1}\right)-H_{1}\right) \\
& +\left[\mathbb{I}-\left(\mathbb{I}-D \mathcal{R}_{1}\left(H_{1}\right)\right) M\right] h \\
& +\widetilde{\mathcal{R}}\left(H_{1}, M h\right)+\left(\mathcal{R}-\mathcal{R}_{1}\right)\left(H_{1}+M h\right) .
\end{aligned}
$$

Denote by $B(r)$ the ball in $\mathcal{B}_{\rho}$ of radius $r$, centered at the origin. One of the goals is to show that $\mathcal{M}$ maps such a ball $B(r)$ into itself, by verifying that

(a) $\left\|\mathcal{R}_{1}\left(H_{1}\right)-H_{1}\right\|_{\rho}=\epsilon \ll r$,

(b) $\quad[\ldots]$ has an operator norm $K<1$,

(c) $\left\|\widetilde{\mathcal{R}}\left(H_{1}, M h\right)+\left(\mathcal{R}-\mathcal{R}_{1}\right)\left(H_{1}+M h\right)\right\|_{\rho} \leq C r^{2}$,

for appropriate constants $\epsilon, K, C>0$. In order to estimate the derivative of $\mathcal{M}$, the bound (c) is extended to $B(2 r)$. Then the derivative of the nonlinear part of $\mathcal{M}$ is bounded by $\mathrm{Cr}$ on $\mathrm{B}(r)$. To be more specific, we estimate

$$
\begin{aligned}
& K_{n}=\left\|\widetilde{\mathcal{R}}\left(H_{1}, M h\right)\right\|_{\rho}, \\
& K_{n}^{\prime}=\left\|\left(\mathcal{R}-\mathcal{R}_{1}\right)\left(H_{1}+M h\right)\right\|_{\rho}, \quad h \in B(n r), \quad n=1,2,
\end{aligned}
$$

in addition to $\epsilon\left(<10^{-14}\right)$ and $K(<0.84)$. Then the existence of a fixed point $H_{*}$ in $B(r)$ follows by verifying that

$$
\epsilon+K r+K_{1}+K_{1}^{\prime}<r, \quad K+\left(K_{2}+K_{2}^{\prime}\right) / r<1 .
$$

The parameters used are $\sigma \approx 0.85001, \kappa \approx \sigma / 0.4, \rho \approx(0.85,0.15)$, and $r \approx 3 * 10^{-12}$. More details can be found in [76]. 


\subsection{Non-twist flows}

Consider Hamiltonians $H: \mathbb{T}^{2} \times \mathbb{R}^{2} \rightarrow \mathbb{R}$ of the form

$$
H(q, p)=\omega \cdot p+h(q, z), \quad z=\Omega \cdot p
$$

where $\omega=\left(\vartheta^{-1}, 1\right)$ and $\Omega=\left(1,-\vartheta^{-1}\right)$, with $\vartheta>0$. The slope on $\mathbb{T}^{2}$ of a solution curve $t \mapsto(q(t), p(t))$ for such a Hamiltonians is given by

$$
\frac{d q_{1}}{d q_{2}}=\frac{\dot{q}_{1}}{\dot{q}_{2}}=\frac{\vartheta^{-1}+\partial_{z} h}{1-\vartheta^{-1} \partial_{z} h}
$$

Assume that there exists an invariant region for the flow, where this slope is bounded away from zero or infinity. If $H$ satisfies the "twist condition" $\partial_{z}^{2} h \neq 0$ in this region, then the slope (4.10) is a monotone function of $z$. Thus, the rotation number $\varrho=\lim _{t \rightarrow \infty} q_{1}(t) / q_{2}(t)$, if it exists, is also a monotone function of $z$. Most KAM-type theorems assume such a twist (or non-degeneracy) condition. It certainly holds for Hamiltonians $K_{1}+$ "small", and the critical Hamiltonian $H_{*}$ most likely satisfies a twist condition near its golden invariant torus.

At the opposite spectrum are the "shearless" tori, where the rotation number is a local minimum or maximum. Such tori appear in many physical systems, including models of the atmosphere, toroidal plasma devices, channel flows, and others [88, 29, 28, 7, 65, 12]. Just like regular KAM tori, a shearless torus with Diophantine rotation number persists under small perturbations (of an integrable system) [32,54]. In fact, they are surprisingly stable. At the point where they break up, they separate two totally chaotic looking regions, while in the twist case, elliptic islands still dominate a non-trivial fraction of phase space.

Apparently, the breakup of such tori is also governed by universality and scaling. In the case of the golden mean and related rotation numbers, numerical investigations of specific two-parameter families $[30,31,3,4]$ reveal self-similarity phenomena, with asymptotic scaling ratios (both in parameter space and phase space) that seem to be independent of the family considered. The self-similarity transformation involves as 12-step shift in the sequence of continued fraction approximants for $\vartheta$, as opposed to the ordinary 1-step shift describing the similarity of periodic orbits in the twist case.

These observations suggests that there exists a "critical" period 12 for an RG transformation like $\mathcal{R}$, in a space of Hamiltonians that permit shearless invariant tori. There is no proof for the existence of such an orbit; nor are there any accurate numerical results, not even from experiments on specific families [30, 31, 3, 4], which usually yield very good values for the universal constants (but only anecdotal evidence for universality). The main problem is that only a few levels of self-similarity can be examined, since each of them involves 12 steps in the continued fraction expansion. A further complication is that two parameters are needed, in order to keep the rotation number of the shearless torus fixed while the nonlinearity is varied. 


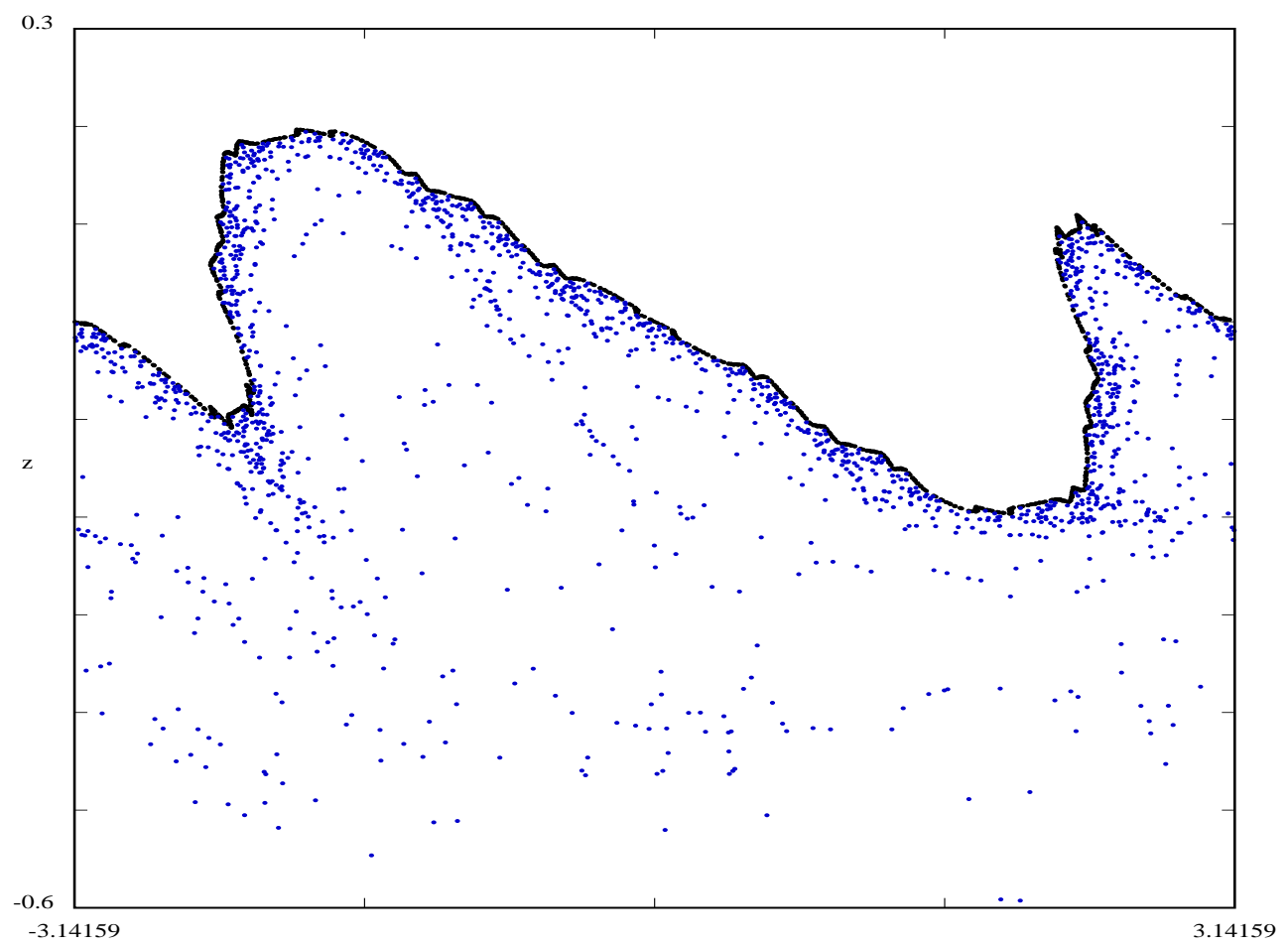

Fig. 5. Orbits for a Hamiltonian with a near-critical shearless golden torus [55]

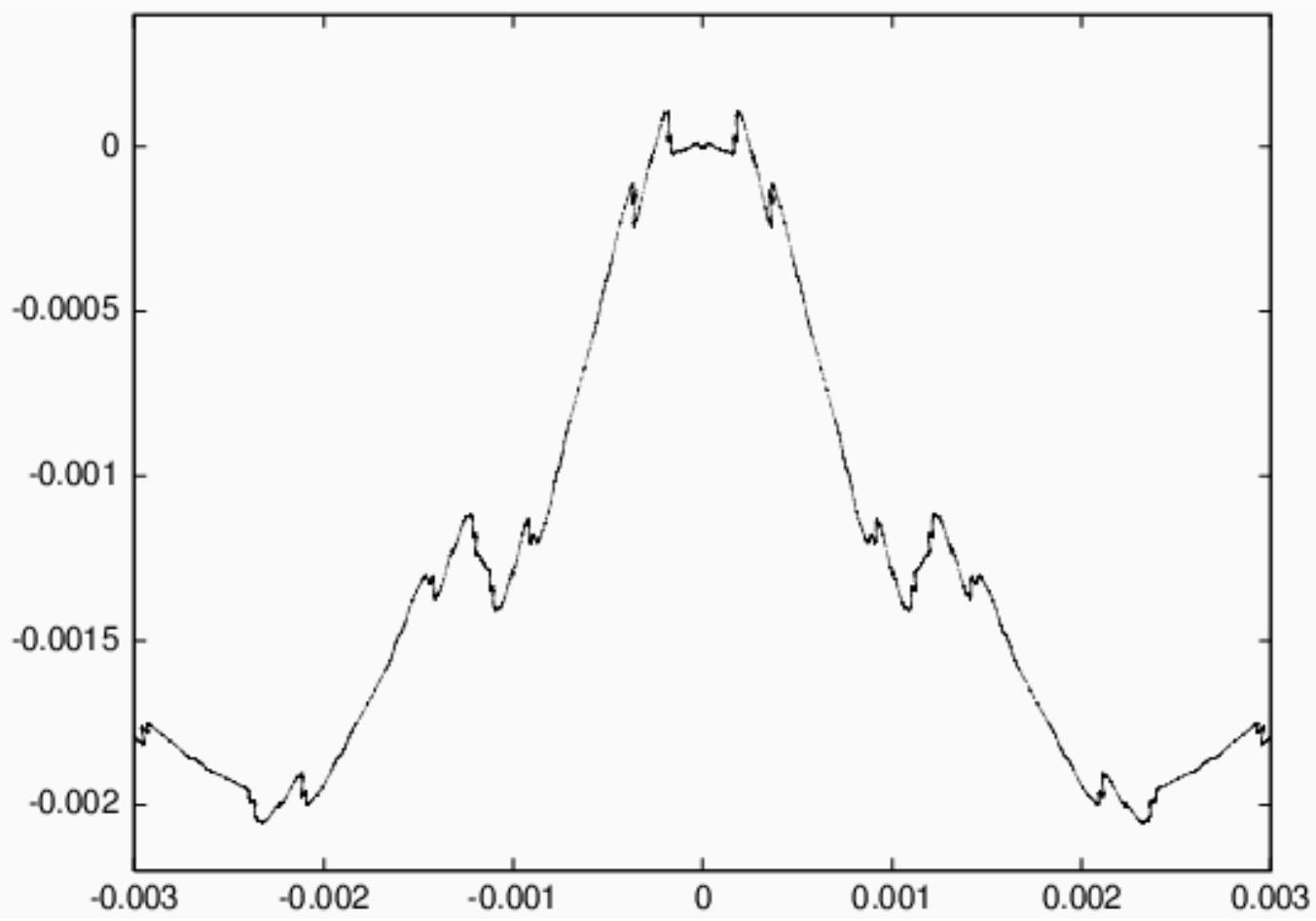

Fig. 6. A critical shearless golden torus [3] 
The following is a short description of a numerical RG analysis that was carried out in [55]. We consider not-so-small perturbations of the two Hamiltonians

$$
K_{\gamma}(q, p)=\omega \cdot p+\gamma(\Omega \cdot p)^{3}, \quad \gamma= \pm \frac{1}{2}
$$

which are a period 2 for the RG transformation $\mathcal{R}$. The invariant torus $p=0$ for $K_{\gamma}$ is shearless: Its rotation number $\vartheta^{-1}$ is maximal if $\gamma \leq 0$, or minimal if $\gamma \geq 0$, among the rotation numbers of nearby orbits. As in the twist case, the analysis is restricted to a subclass of Hamiltonians $H$ with a suitable symmetry. The symmetry chosen in this case is

$$
-H=\mathcal{J} H=H \circ J, \quad J(q, p)=(-q,-p) .
$$

Its usefulness stems from the fact that if $-\mathcal{J} H=H$, then the orbit of the origin for $H$ is invariant under $J$. Thus, an invariant torus passing through the origin divides the energy surface $H=H(0,0)$ into symmetric halves, and so the torus has to be shearless.

More specifically, consider Hamiltonians

$$
H(q, p)=\omega \cdot p+h(q, z), \quad h(q, z)=\sum_{\nu, k} h_{\nu, k} e^{i \nu \cdot k} z^{k}, \quad z=\Omega \cdot p
$$

that are odd under $\mathcal{J}$, and real-valued for real arguments. In the numerical implementation, these Hamiltonians $H$, and all other functions that appear in the definition of $\mathcal{R}(H)$, are truncated to $|\nu| \leq N_{1}, k \leq N_{2},|\nu|+k \leq N_{3}$, and represented as finite arrays of Fourier-Taylor coefficients. Implicit Equations like are solved by Newton's method. After eliminating one of the unstable directions of $\mathcal{R}$, we searched for points on the stable manifold of the expected period $H_{0}, H_{1}, \ldots, H_{11}$, using a bisection algorithm along lines through $K_{\gamma}$. One of the first findings was an apparent additional symmetry

$$
\left\{\mathcal{J}_{\alpha} H_{n}: \alpha \in A\right\}=\left\{-H_{n},-H_{m}, \mathcal{J}_{0} H_{m}\right\}, \quad m=n+6(\bmod 12),
$$

where $A=\{(1,0),(0,1),(1,1)\}$ and

$$
\mathcal{J}_{\alpha} H=H \circ J_{\alpha}, \quad J_{\alpha}(q, p)=(q+\pi \alpha,-p) .
$$

If correct, then $H_{0}, H_{1}, \ldots, H_{5}$ are in fact a period 6 for the transformation $\mathcal{N}=\mathcal{J}_{\alpha} \circ \mathcal{R}$. Iterating $\mathcal{N}$ along the presumed stable manifold of $\mathcal{N}$ at the critical period 6 , bisecting again when necessary, and starting all over with different choices for the RG parameters $\sigma, \kappa$, when things failed to converge (an extremely tedious procedure), an approximate fixed point for $\mathcal{N}^{6}$ was found. Our numerical values for the expanding eigenvalues $\delta_{j}$ of $\mathcal{N}$, and for the average scaling $\bar{\mu}$, defined by $\bar{\mu}^{6}=\mu\left(H_{0}\right) \mu\left(H_{1}\right) \cdots \mu\left(H_{5}\right)$, are

$$
\delta_{1}^{1 / 6} \approx 2.661, \quad \delta_{2}^{1 / 6} \approx 1.585, \quad \bar{\mu} \approx 0.3659
$$

For comparison, the numerically observed values for $\delta_{1}^{1 / 6}$ and $\delta_{2}^{1 / 6}$, computed from the data in [3] are 2.678 and 1.583. This supports the idea that the breakup of shearless golden tori is governed by a RG period 12 , but it is clear that much more work is necessary. 
It is also possible to give some "explanation" why a RG period 6 or 12 should be expected in connection with shearless invariant tori. The same argument for rotation numbers in $Q[\vartheta]$, with $\vartheta=\left(\sqrt{n^{2}+4}+n\right) / 2$, suggests that the corresponding RG transformation should have a period 6 or 12 if $n$ is odd, and a period 4 or 8 if $n$ is even. More details can be found in [55].

\section{Invariant tori}

A canonical application of renormalization (for flows) is the construction of invariant tori or other conjugacies. The basic version discussed here applies to self-similar frequency vectors and involves a single $\mathrm{RG}$ transformation $\mathcal{R}$, a fixed point for $\mathcal{R}$, and its stable manifold $\mathcal{W}^{s}$. However, the procedure extends easily Diophantine rotation vectors, once we generalize the notion of a stable manifold to sequences of RG transformations. In this section we also discuss critical (non-differentiable) and shearless invariant tori.

\subsection{Some ideas and results}

Consider the RG transformation for some fixed but arbitrary class of vector fields $X$,

$$
\mathcal{R}(X)=\eta^{-1} \Lambda_{X}^{*} X, \quad \Lambda_{X}=\mathcal{T}_{\mu} \circ \mathcal{U}_{X}
$$

We note that, by construction, $\mathcal{U}_{c X}=\mathcal{U}_{X}$ for nonzero constants $c$. Assume that $X_{0}$ lies on the stable manifold of some fixed point $X_{\infty}$. Then the sequence of vector fields $X_{n}=\mathcal{R}^{n}\left(X_{0}\right)$ converges to $X_{\infty}$. Combining the $n$ RG steps connecting $X_{n}$ to $X_{0}$ yields

$$
X_{n}=\eta_{1}^{-1} \cdots \eta_{n}^{-1}\left(\Lambda_{X_{\infty}}^{n}\right)^{*} \circ \mathcal{V}_{n-1}^{*} \circ \ldots \circ \mathcal{V}_{0}^{*} X_{0}
$$

where

$$
\mathcal{V}_{k}=\Lambda_{X_{\infty}}^{k} \circ \Lambda_{X_{k}} \circ \Lambda_{X_{\infty}}^{-k-1}
$$

Assuming that $\Lambda_{X_{\infty}}$ is one-to-one, we have $\eta_{\infty}\left(\Lambda_{X_{\infty}}^{*}\right)^{-1} X_{\infty}=X_{\infty}$ on the range of $\Lambda_{X_{\infty}}$. The goal now is to show that $\prod_{k}\left(\eta_{k} / \eta_{\infty}\right) \rightarrow c$, and $\mathcal{V}_{k} \rightarrow$ I sufficiently fast, such that

$$
\mathcal{V}_{0} \circ \mathcal{V}_{1} \circ \ldots \circ \mathcal{V}_{n-1} \rightarrow \Gamma
$$

in some appropriate topology. If the limit (5.4) exists on a nontrivial part of phase space, then

$$
\Gamma^{*} X_{0}=c X_{\infty}
$$

In this case, the construction yields a conjugacy to a constant vector field, for every $X$ on the stable manifold of the fixed point $X_{\infty}$. This can be applied e.g. to parametrized families of vector fields $\beta \mapsto X^{\beta}$, under conditions that guarantee that the family intersects the stable manifold of $\mathcal{R}$. A procedure of this type for skew flows is described in Subsection 8.3 .

For Hamiltonian vector fields on $\mathbb{T}^{d} \times \mathbb{R}^{d}$, the limit (5.4) can be expected to exist only on $\mathbb{T}^{d} \times\{0\}$. In this case, $\Gamma$ is an invariant torus for $H$. Concerning uniqueness, recall 
that invariant tori with given rotation numbers typically come in one-parameter families, indexed e.g. by the energy. The tori $\Gamma$ constructed here are characterized by the property that the integrals

$$
A(\gamma)=\frac{1}{2 \pi} \oint_{\gamma} \sum_{j=1}^{d} p_{j} d q_{j}
$$

vanish along each closed curve $\gamma$ on the torus. Tori with this property will be referred to as being "centered at $p=0$ ". This property is invariant under symplectic maps (1.6) obtained from generating functions (1.7). Thus, since $\Gamma$ is a limit of such maps, with the $p$-domain shrinking to the point zero, $\Gamma$ is centered at $p=0$.

If $H$ does not lie on the stable manifold, but satisfies a suitable nondegeneracy condition, then the procedure sketched above may still be used to construct an invariant torus for $H$. To be more specific, consider the RG transformation $\mathcal{R}$ for analytic Hamiltonians, where

$$
\mathcal{R}(H)=\frac{1}{\eta \mu} H \circ \Lambda_{H},
$$

with $\Lambda_{H}=\mathcal{T}_{\mu} \circ \mathcal{U}_{H^{\prime}}$ and $H^{\prime}=H \circ \mathcal{T}_{\mu}$. As can be computed explicitly (see Subsection 6.1), the expanding subspace for $D \mathcal{R}\left(K_{0}\right)$ at the trivial fixed point $K_{0}(q, p)=\omega \cdot p$, consist of all functions $h_{v}(q, p)=v \cdot p$, with $v$ in the contracting subspace $V$ of the matrix $T$. Consider now an analytic Hamiltonian

$$
K(q, p)=\omega \cdot p+\frac{1}{2} p \cdot M p+f(p), \quad f(p)=\mathcal{O}\left(|p|^{3}\right),
$$

where $M$ is a real symmetric $d \times d$ matrix, such that the quadratic form $p \mapsto p \cdot M p$ is nondegenerate when restricted to the $d-1$ dimensional contracting subspace of $T^{*}$. We may assume that $h$ is small, since this can always be achieved with a scaling $K \mapsto s^{-1} K(., s$.$) .$ Then the family $v \mapsto K(q, p+v)$, indexed by $V$, is transversal to the local stable manifold $\mathcal{W}^{s}$ of $\mathcal{R}$. Thus, if $H$ is an analytic Hamiltonian close to $K$, then the family $v \mapsto H(q, p+v)$ intersects $\mathcal{W}^{s}$ at exactly one point, say for $v=v_{*}$.

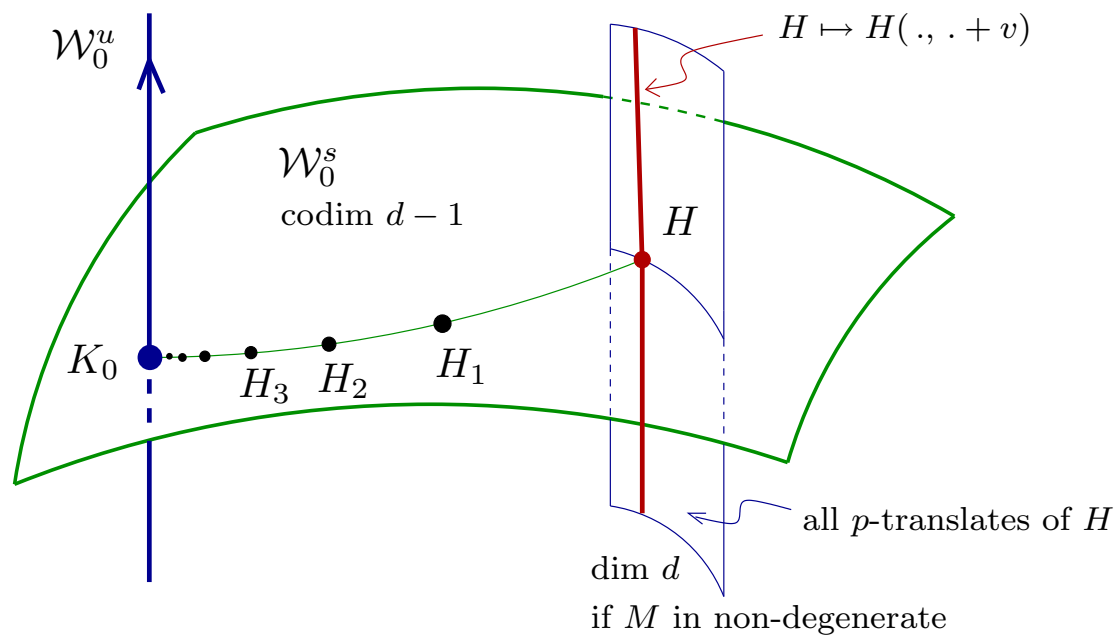

Fig. 7. A Hamiltonian and its $p$-translates 
Clearly, if $H\left(., .+v_{*}\right)$ has an invariant torus with rotation vector $c \omega$, then so does $H$.

One of the issues with an RG based construction of invariant tori is the loss of regularity that occurs in the process. This concerns mostly regularity in the angular variable $q$. To simplify the discussion, consider again the RG transformation (5.7) near the trivial fixed point $K_{0}(q, p)=\omega \cdot p$. Then the equation (5.3) becomes

$$
\mathcal{V}_{k}=\mathcal{T}_{\mu_{\infty}}^{k} \circ \Lambda_{H_{k}} \circ \mathcal{T}_{\mu_{\infty}}^{-k-1}
$$

Notice that $T$ has $d-1$ contracting directions. Thus, the composition with $\mathcal{T}_{\mu_{\infty}}^{-k-1}$ shrinks the domain of analyticity in the contracting directions of $T$, down to zero in the limit $k \rightarrow \infty$. However, the non-integrable part of $H_{n}$, which determines the size of $\mathcal{U}_{H_{n}}-\mathrm{I}$, tends to zero even more rapidly. This fact can be used to control some fixed number of $q$-derivatives of $\mathcal{V}_{k}$, and to prove that the torus $\Gamma$ has that many continuous derivatives. This result is far from optimal, though. These tori are known to be analytic.

Fortunately, the analyticity of $\Gamma=\Gamma_{H}$ for near-integrable Hamiltonians $H$ can be obtained in a different way. (And this works not only for Hamiltonian flows.) Consider torus-translations $J_{v}(q, p)=(q+v, p)$. First, one verifies that for real $v$,

$$
\mathcal{R} \circ \mathcal{J}_{v}=\mathcal{J}_{T^{-1} v} \circ \mathcal{R}, \quad \mathcal{J}_{v} H=H \circ J_{v}
$$

This is an explicit computation, using that $\mathbb{I}^{ \pm}$commutes with $\mathcal{J}_{v}$, and that $\mathcal{J}_{v}$ is an isometry on the spaces $\mathcal{F}_{\rho}$. The equation (5.10) shows in particular that the local stable manifold of $\mathcal{R}$ (at $K_{0}$ ) is invariant under $\mathcal{J}_{v}$. Here, one uses the fact that the trivial fixed point is invariant under translations on $\mathbb{T}^{d}$. Assume now that $H$ belongs to this local stable manifold. Then the second step is to verify that the construction of $\Gamma=\Gamma_{H}$ yields

$$
\Gamma_{H \circ J_{v}}=J_{v}^{-1} \circ \Gamma_{H} \circ J_{v}
$$

for real translations $v$. This identity implies that

$$
\Gamma_{H}(q)=E_{q} \Gamma_{H}=E_{0}\left(\Gamma_{H} \circ J_{q}\right)=J_{q} E_{0} \Gamma_{H \circ J_{q}} .
$$

where $E_{q}$ denotes the evaluation functional $f \mapsto f(q)$. Now, it suffices to prove that the map $H \mapsto \Gamma_{H}$ is analytic in an open neighborhood of $K_{0}$ in $\mathcal{H}_{\rho}=K_{0}+\mathcal{F}_{\rho}$. Then, if $H$ is sufficiently close to $K_{0}$ in a space $\mathcal{H}_{\rho^{\prime}}$ with $\rho_{1}^{\prime}>\rho_{1}$, then $q \mapsto H \circ J_{q}$ is an analytic family in the domain of $H \mapsto \Gamma_{H}$. Thus the right hand side of equation (5.11) depends analytically on $q$, defining an analytic continuation of the left hand side. It should be mentioned that the analyticity of $H \mapsto \Gamma_{H}$ is a consequence of the explicit construction of $\Gamma_{H}$ via uniformly converging limits of analytic maps.

A construction along these lines, of analytic invariant tori with self-similar frequency vectors, can be found in [74]. In the case $d=2$, the procedure was generalized by Kocić [81] to frequency vectors $\omega$ that satisfy a Diophantine condition (with restrictions on the Diophantine exponent), using a sequence of RG transformation $\mathcal{R}_{n}$ associated with the continued fraction expansion of the rotation number $\omega_{1} / \omega_{2}$. Recent work by Marklov, Lopes Dias, and Khanin [70] extends this construction to arbitrary Diophantine vectors $\omega$ 
in dimensions $d \geq 2$, after introducing an appropriate multidimensional continued fraction expansion. This expansion, and a RG analysis of skew flows [79] that is based on it, will be described in Sections 7 and 8. It should be noted that for Hamiltonians $H(q, p)$ that are linear in $p$, there is no domain loss in the construction of $\Gamma$. In this case, $\Gamma$ is a proper change of variables that reduces $H$ to a constant multiple of $K_{0}$. This can be applied to vector fields on $\mathbb{T}^{d}$, by considering the associated Hamiltonian $H(q, p)=p \cdot X(q)$. A RG analysis of flows on $\mathbb{T}^{2}$ with Brjuno rotation numbers was given in [96].

A RG analysis of one-parameter families of Hamiltonians with a pair of (golden) invariant tori bifurcating from a shearless invariant torus was carried out by Gaidashev in [54]. A brief description of this work, which involves two different fixed points of $\mathcal{R}$, will be given in Subsection 5.5.

In all these cases, invariant tori were constructed for vector fields that are close to constant, i.e., the RG fixed point used is a linear vector field, or in the Hamiltonian case, one of the integrable Hamiltonians $K_{0}$ or $K_{\gamma}$ discussed earlier. Invariant tori for critical Hamiltonians near the nontrivial fixed point $H_{*}$ are discussed below. This includes a description of the proof of

Theorem 5.1. [77] In some open neighborhood of $H_{\infty}$, every Hamiltonian that lies on the local (strong) stable manifold of $\mathcal{R}$ has a non-differentiable golden invariant torus.

Numerical results indicate that $\mathcal{R}$ is hyperbolic at $H_{\infty}$, with a single relevant expanding direction; but this has not yet been proved. As mentioned in Subsection 1.6, the Hamiltonians on the non-KAM side of the stable manifold are expected to have hyperbolic orbits with golden mean rotation number. A possible approach for proving a local version of this conjecture is described in [27].

\subsection{Renormalization of invariant tori}

As was shown above, no generality is lost by using spaces like $C^{r}$ for the construction of the invariant torus $\Gamma$. In most of the work that have been mentioned, the differential version (1.3) of the torus equation

$$
\Gamma_{H} \circ \Psi^{t}=\Phi_{H}^{t} \circ \Gamma_{H}, \quad \Psi^{t}(q, 0)=(q+t \omega, 0),
$$

was used. This requires at least $r \geq 1$.

Some recent work $[77,81,78]$ follows a different approach, based entirely on semiconjugacies, where it is natural to use equation (5.13) directly. So there is no need to work with differentiable functions. We will now describe the construction given in [77] of invariant tori for Hamiltonians on the local stable manifold of the nontrivial fixed point $H_{*}$ described in Theorem 4.1. In this case, the argument that was used above to show that a continuous torus $\Gamma$ has to be analytic, fails, since the fixed point $H_{*}$ is not invariant under torus-translations $\mathcal{J}_{v}$. In fact, one of our goals is to show that the Hamiltonians on the local stable manifold of $\mathcal{R}$ at $H_{*}$ are "critical", in the sense that their golden invariant tori are not differentiable. This argument uses specific properties of the fixed point $H_{*}$ and of its scaling map $\Lambda_{H_{*}}$. The other steps in the construction, which we discuss first, can also be adapted to the study of near-integrable Hamiltonians [81]. Notice that the RG 
transformation used near $H_{*}$ is also of the form (5.7), but with $\Lambda_{H}=\mathcal{T}_{\mu} \circ U_{\phi_{0}} \circ \mathcal{U}_{H^{\prime}}$ and $H^{\prime}=H \circ \mathcal{T}_{\mu} \circ U_{\phi_{0}}$.

The basic idea is to relate the equation (5.13) to the analogous equation for the renormalized Hamiltonian $\widetilde{H}=\mathcal{R}(H)$. For the two flows, we have

$$
\Lambda_{H} \circ \widetilde{\Phi}^{t}=\Phi^{\eta^{-1} t} \circ \Lambda_{H}
$$

with $\eta$ depending on $H$ (in general). This identity follows from the fact that $\mathcal{T} \circ U_{\phi_{0}} \circ \mathcal{U}_{H}$ is symplectic, and from an explicit computation, showing that (5.14) holds in the case where $\widetilde{H}(q, p)$ is taken to be $\eta^{-1} \mu^{-1} H(q, \mu p)$. Now consider Hamiltonians of the form $H(q, p)=\omega \cdot p+h(q, \Omega \cdot p)$ with $\Omega \cdot \omega=0$. Then the time renormalization is $\eta=\vartheta^{-1}$, where $\vartheta$ is the eigenvalue of $T$ for the eigenvector $\omega$. Define

$$
\mathcal{M}_{H}(f)=\Lambda_{H} \circ f \circ \mathcal{T}^{-1}
$$

for any map $f$ from $\mathbb{T}^{d} \times\{0\}$ to the domain of $\Lambda_{H}$. Assume that $\widetilde{\Gamma}$ is an invariant torus for $\widetilde{H}$, taking values in the domain of $\Lambda_{H}$, and set $\Gamma=\mathcal{M}_{H}(\widetilde{\Gamma})$. Then by using the identity (5.14), together with the fact that $\mathcal{T} \circ \Psi^{t}=\Psi^{\vartheta t} \circ \mathcal{T}$, we obtain

$$
\begin{aligned}
\Gamma \circ \Psi^{t} & =\Lambda_{H} \circ \widetilde{\Gamma} \circ \mathcal{T}^{-1} \circ \Psi^{t}=\Lambda_{H} \circ \widetilde{\Gamma} \circ \Psi^{\vartheta^{-1}} t \circ \mathcal{T}^{-1} \\
& =\Lambda_{H} \circ \widetilde{\Phi}^{\vartheta^{-1} t} \circ \widetilde{\Gamma} \circ \mathcal{T}^{-1}=\Phi^{t} \circ \Lambda_{H} \circ \widetilde{\Gamma} \circ \mathcal{T}^{-1}=\Phi^{t} \circ \Gamma
\end{aligned}
$$

This shows that $\Gamma$ is an invariant torus for $H$.

It also suggest that an invariant torus for a fixed point $H$ of $\mathcal{R}$ is a fixed point of $\mathcal{M}_{H}$. Such a torus $\Gamma$ satisfies the equation $\Gamma \circ \mathcal{T}=\Lambda_{H} \circ \Gamma$, which, together with purely topological arguments, can be used to prove the following.

Lemma 5.2. [77] Let $H$ be a fixed point of $\mathcal{R}$, and let $\Gamma$ be an invariant torus for $H$ that is continuous and homotopic to $\mathcal{T}$. If $\Gamma$ is a fixed point for $\mathcal{M}_{H}$, then $\Lambda_{H}$ maps range $(\Gamma)$ invertibly onto itself.

Let now $H_{\infty}$ be a fixed point of $\mathcal{R}$ with a well defined local (strong) stable manifold $\mathcal{W}^{s}$. Examples are the integrable fixed points (2.5) and $K_{0}$, and for $d=2$, the nontrivial fixed point $H_{*}$. Given any Hamiltonian $H_{0} \in \mathcal{W}^{s}$, define $H_{n}=\mathcal{R}^{n}\left(H_{0}\right)$. In order to simplify notation, we will write $\Lambda_{n}$ and $\mathcal{M}_{n}$ in place of $\Lambda_{H_{n}}$ and $\mathcal{M}_{H_{n}}$, respectively.

Our first goal is to show that $\mathcal{M}_{H}$ is a contraction on some appropriate space of maps, whenever $H$ is sufficiently close to $H_{\infty}$. If we knew e.g. that each of the Hamiltonians $H_{n}$ had a unique invariant torus $\Gamma_{n}$ (centered at $p=0$ ) with frequency vector $\omega$, then these tori should satisfy

$$
\Gamma_{n}=\mathcal{M}_{n}\left(\Gamma_{n+1}\right)=\Lambda_{n} \circ \Gamma_{n+1} \circ \mathcal{T}^{-1}, \quad n=0,1,2, \ldots
$$

This argument can in fact be turned around: We will use the contraction property of the maps $\mathcal{M}_{n}$ to construct, via inverse limit, a sequence of maps $\Gamma_{n}$ satisfying (5.16). Then we will show that $\Gamma_{n}$ is indeed an invariant torus for $H_{n}$. 


\subsection{Existence}

In what follows, we will restrict our analysis to a special case. Let $\omega=\left(\vartheta^{-1}, 1\right)$ and $\Omega=$ $\left(1,-\vartheta^{-1}\right)$, with $\vartheta$ the golden mean. Let $\omega^{\prime}=c \omega$ and $\Omega^{\prime}=c \omega$, such that $\omega^{\prime} \cdot \omega=\Omega^{\prime} \cdot \Omega=1$. We consider only Hamiltonians of the form $H(q, p)=\omega \cdot p+h(q, z)$, where $z=\Omega \cdot p$. As a result, only coordinate changes of the form

$$
U=\mathrm{I}+u, \quad u(q, p)=\left(u_{x} \Omega, u_{y} \omega^{\prime}+u_{z} \Omega^{\prime}\right)
$$

need to be considered. Just like the flow for $H$, they are "time preserving" in the sense that $\tau=\omega^{\prime} \cdot q$ is invariant. Imposing further that $h(q, z)$ be even in $q$, we can restrict $u_{x}$ to be odd in $q$, and $u_{z}$ even. This will be referred to as the "parity preserving" property. In addition, $u(q, p)$ only needs to depend on $q$ and $z$, but not on $y=\omega \cdot p$. As a result, $u_{y}$ enters trivially in any composition of such transformations. Thus, in order to simplify notation, we will identify $u$ with $\left(u_{x}, u_{z}\right)$. For the same reason, $u$ and $U$ will be identified with functions of the variables $(q, z)$. The same applies to the functions $\Gamma=\mathrm{I}+\gamma$ that appear in the construction of invariant tori. In addition, these functions do not depend on $z$.

A connection between the transformation $\mathcal{R}$ for time preserving Hamiltonian flows and the RG transformation for commuting maps can be found in [75]. Next, we introduce some functions spaces. All of the functions that need to be considered here are either even or odd in $q$. The coefficients in the cosine (sine) series of an even (odd) function $f=f(q)$ will be denoted by $f_{\nu}$. For functions like $\gamma_{x}$ and $\gamma_{z}$, we will use the space $\mathcal{B}_{0}$ (if even) or $\mathcal{C}_{0}$ (if odd) of functions $f: D \rightarrow \mathbb{C}$ with finite norm

$$
\|f\|_{0}=\sum_{\nu}\left|f_{\nu}\right| e^{\delta|\omega \cdot \nu|}(1+|\Omega \cdot \nu|)^{r} .
$$

Here $\delta, r<1$ are small positive numbers to be chosen later. The space $\mathcal{C}_{0} \times \mathcal{B}_{0}$, equipped with the norm $\left\|\left(f_{x}, f_{z}\right)\right\|_{0}=\max \left\{\left\|f_{x}\right\|_{0}, 2\left\|f_{z}\right\|_{0}\right\}$, will be denoted by $\mathcal{A}_{0}$.

Consider now the transformation $\mathcal{M}_{H}$ defined earlier,

$$
\mathcal{M}_{H}(\Gamma)=\Lambda_{H} \circ \Gamma \circ \mathcal{T}^{-1} .
$$

Concerning the composition on the right by $\mathcal{T}^{-1}$, notice that $f \mapsto f \circ T^{-1}$ is bounded on $\mathcal{F}_{0}$, with operator norm close to 1 if $r>0$ is small. For the composition of the right by $\Lambda_{H}$, consider first $H=K_{0}$. Then $\Lambda_{H}$ acts on $\gamma$ by multiplying $\gamma_{x}$ by $-\vartheta^{-1}$, and $\gamma_{z}$ by $-\vartheta^{-2}$. Both of these numbers are less than $2 / 3$ in modulus. Thus, $\mathcal{M}_{H}$ is a contraction on $\mathcal{A}_{0}$ in this case, if $r>0$ is sufficiently small. This clearly remains true for analytic Hamiltonians near $K_{0}$.

The proposition below implies that the same holds for Hamiltonians near the fixed point $H_{*}$. Given $\rho=\left(\rho_{x}, \rho_{z}\right)$ with positive components, define $D_{\rho}$ to be the complex neighborhood of $D=\mathbb{T}^{2} \times\{0\}$, characterized by $|\operatorname{Im} \tau|<\delta,|\operatorname{Im} x|<\rho_{x}$ and $|z|<\rho_{z}$. Here, $x=\Omega^{\prime} \cdot q$. Let $\mathcal{B}_{\rho}$ be the space of analytic functions $h: D_{\rho} \rightarrow \mathbb{C}$ that are even in $q$ and have finite norm

$$
\|h\|_{\rho}=\sum_{\nu, k}\left|h_{\nu, k}\right| e^{\delta|\omega \cdot \nu|} \cosh \left(\rho_{x} \Omega \cdot \nu\right) \rho_{z}^{k},
$$


where $h_{\nu, k}$ are the coefficients in the cosine-Taylor expansion of $h$. The affine space $K_{0}+\mathcal{B}_{\rho}$ will be denoted by $\mathcal{H}_{\rho}$. In what follows, we use the same domain and norm parameters as in [76], namely $\rho \approx(0.85,0.15)$ and $r \approx 10^{-4}$. Let $\rho^{\prime}=\left(\rho_{x}, \rho_{z} / 2\right)$.

Proposition 5.3. [77] If $\delta>0$ is chosen sufficiently small, then there exists a bounded open neighborhood $V$ of $H_{\infty}$ in $\mathcal{H}_{\varrho}$, such that the following holds. The map $H \mapsto \Lambda_{H}$ is analytic on $V$, and there exists a positive $a<1$, an open ball $B$ in $\mathcal{A}_{0}$, and a concentric closed ball $B_{0} \subset B$, such that for every $H \in V$, the transformation $\mathcal{M}_{H}$ is well defined on $B$, maps $B$ into $B_{0}$, and contracts distances by a factor $\leq a$. The range of any function $\Gamma \in B$ is contained in $D_{\rho^{\prime}}$. Furthermore, $(H, \Gamma) \mapsto \mathcal{M}_{H}(\Gamma)$ is analytic on $V \times B$.

The proof in is based on computer-assisted estimates for the scaling map $\Lambda_{H_{*}}$. After determining (numerically) an approximate fixed point $f$ for $\mathcal{M}_{H_{*}}$, the stated properties were obtained by estimating the norm of $\mathcal{M}_{H_{*}}(f)-f$ and of the derivative of $\mathcal{M}_{H_{*}}$ near $f$. Notice that Proposition 5.3 the existence of a fixed point $\Gamma_{\infty}$ for the map $\mathcal{M}_{H_{*}}$.

An analogous proposition for the trivial fixed point $K_{0}$ follows from our earlier discussion, together with the fact that $\mathcal{R}$ is analytic near $K_{0}$.

Now we are ready to construct a sequence of functions $\left\{\Gamma_{n}\right\}$ that satisfy equation (5.16). Consider either $H_{\infty}=K_{0}$ or $H_{\infty}=\mathcal{H}_{*}$. Let $V \subset \mathcal{H}_{\varrho}$ and $B \subset \mathcal{A}_{0}$ be as in Proposition 5.3 , and let $F_{0}, F_{1}, \ldots$ be arbitrary maps in $B$. Given a sequence of Hamiltonians $H_{0}, H_{1}, \ldots$ in $V$, define

$$
\begin{aligned}
\Gamma_{n, m} & =\left(\mathcal{M}_{n} \circ \mathcal{M}_{n+1} \circ \ldots \circ \mathcal{M}_{m-1}\right)\left(F_{m}\right) \\
& =\Lambda_{n} \circ \Lambda_{n+1} \circ \ldots \circ \Lambda_{m-1} \circ F_{m} \circ \mathcal{T}^{-m+n}, \quad 0 \leq n<m .
\end{aligned}
$$

Theorem 5.4. [77] There is a constant $C>0$ such that the following holds. Let $H_{0}, H_{1}, \ldots$ be Hamiltonians in $V$. Then the limits $\Gamma_{n}=\lim _{m \rightarrow \infty} \Gamma_{n, m}$ exist in $\mathcal{A}_{0}$, are time and parity preserving, do not depend on the choice of the maps $F_{m}$, and satisfy the bounds

$$
\left\|\Gamma_{n}-\Gamma_{\infty}\right\|_{0} \leq C \sup _{m \geq n}\left\|H_{m}-H_{\infty}\right\|_{\varrho}, \quad n=0,1, \ldots
$$

If $H_{n}=\mathcal{R}^{n}\left(H_{0}\right)$ for all $n>0$, then (5.16) holds. If in addition, $V$ has been chosen sufficiently small, and $H_{0}$ belongs to $\mathcal{W}^{s}$, then $\Gamma_{n}$ is a golden invariant torus for $H_{n}$, for each $n \geq 0$.

The main ingredients in the proof of this theorem are the following.

Proposition 5.3 shows that if $n<m<k$, then the difference $\Gamma_{n, k}-\Gamma_{n, m}$ is bounded in norm by $d a^{m-n}$, where $d$ is the diameter of $B$. Thus, the sequence $m \mapsto \Gamma_{n, m}$ converges in $\mathcal{A}_{0}$ to a limit $\Gamma_{n}$, and this limit is independent of the choice of the maps $F_{m}$. The estimate (5.22) is obtained by using the analyticity of the map $H_{0} \mapsto \Gamma_{0}$.

Assume now $H_{n}=\mathcal{R}^{n}\left(H_{0}\right)$ for all $n>0$. Then (5.16) is obtained by taking $F_{m}=\Gamma_{m}$ for all $m$, and using that the maps $\mathcal{M}_{n}$ are continuous. In order to prove that $\Gamma_{n}$ is an invariant torus for $H_{n}$, we can use the identity

$$
\begin{aligned}
\Phi_{n}^{t} \circ \Gamma_{n, m} \circ \Psi^{-t} & =\Lambda_{n} \circ \Lambda_{n+1} \circ \ldots \circ \Lambda_{m-1} \circ \Phi_{m}^{t_{m-n}} \circ \Gamma_{\infty} \circ \Psi^{-t_{m-n}} \circ \mathcal{T}^{-m+n} \\
& =\left(\mathcal{M}_{n} \circ \mathcal{M}_{n+1} \circ \ldots \circ \mathcal{M}_{m-1}\right)\left[\Phi_{m}^{t_{m-n}} \circ \Gamma_{\infty} \circ \Psi^{-t_{m-n}}\right],
\end{aligned}
$$


where $t_{k}=\vartheta^{-k} t$, and where $\Psi_{k}$ denotes the flow for $H_{k}$. Here, we have used that $\mathcal{T} \circ \Phi^{s}=\Phi^{\vartheta s} \circ \mathcal{T}$, Notice that the map [...] in equation (5.23) is time preserving, since the two flows change $\tau$ by opposite amounts. Thus, equation (5.23) is an identity between maps in $\mathcal{A}_{0}$.

Consider first the case where $H_{n}=H_{\infty}$ for all $n \geq 0$. Then the two flows in [...] tend to the identity as $m \rightarrow \infty$, Thus, [...] takes values in $D_{\rho}$ for large $m$. As $m \rightarrow \infty$, both sides converge in $\mathcal{A}_{0}$ to $\Gamma_{n}$. But convergence in $\mathcal{A}_{0}$ implies pointwise convergence, and since $\Psi_{n}^{t}$ and $\Phi^{t}$ are both continuous and invertible, we conclude that $\Psi_{n}^{t} \circ \Gamma_{n} \circ \Phi^{-t}=\Gamma_{n}$. This shows that $\Gamma_{n}$ is a golden invariant torus for $H_{n}$.

For general $H_{0} \in \mathcal{W}^{s}$ near $H_{\infty}$,we can now use that

$$
\Phi_{m}^{t_{m-n}} \circ \Gamma_{\infty} \circ \Psi^{-t_{m-n}}=\Phi_{m}^{t_{m-n}} \circ \Phi_{\infty}^{-t_{m-n}} \circ \Gamma_{\infty}
$$

Again, the map [...] in equation (5.23) takes values in $D_{\rho}$ for large $m$. So by the same argument as above, $\Gamma_{n}$ is a golden invariant torus for $H_{n}$.

\subsection{Critical invariant tori}

The invariant torus $\Gamma_{\infty}$ for a fixed point $H_{\infty}$ of $\mathcal{R}$ satisfies the equation

$$
\Lambda_{\infty} \circ \Gamma_{\infty}=\Gamma_{\infty} \circ \mathcal{T}
$$

This shows e.g. that $\left(0, p_{\infty}\right)=\Gamma_{\infty}(0)$ is a fixed point of the scaling map $\Lambda_{\infty}$. If $\Gamma_{\infty}$ is smooth and one-to-one and near this fixed point, then the semi-conjugacy (5.25) suggests that the eigenvalues of $T$ are also eigenvalues of $D \Lambda_{\infty}\left(0, p_{\infty}\right)$. This is trivially the case for the fixed point $K_{0}$, whose scaling map is $\mathcal{T}_{\mu}$, with $\mu=\vartheta^{-3}$. The eigenvalues of $\mathcal{T}_{\mu}$ are $\vartheta,-\vartheta^{-1}, \vartheta^{-4}$, and $-\vartheta^{-2}$. These are the trivial values of the scaling constants $\lambda_{\tau}, \lambda_{x}$, $\lambda_{y}$, and $\lambda_{z}$, whose critical values we shall now describe. First, we note that if $U$ is parity preserving, then the line $q=0$ is invariant under $U$. Thus, the maps $\Lambda_{H}$ are of the form

$$
\Lambda_{H}(0, z)=\left(0, \ell_{H}(z)\right)
$$

where $\ell_{H}$ is some function of one variable.

The following proposition concern the fixed point $H_{*}$ described in Theorem 4.1. Its proof is computer-assisted.

Proposition 5.5. [77] The (analytic) function $\ell_{H_{*}}$ maps the interval $\left[-\varrho_{z} / 2, \varrho_{z} / 2\right]$ into its interior, has a globally attracting fixed point $z_{\infty}$, and the derivative of $\ell_{H_{*}}$ at this fixed point is $\lambda_{z}=-0.326063 \ldots$. Furthermore, $\mu\left(H_{*}\right)=\mu_{\infty}=0.230460196 \ldots$.

One of the conclusions is that $\Lambda_{H_{*}}$ has a fixed point $(q, p)=\left(0, p_{\infty}\right)$ with $\Omega \cdot p_{\infty}=z_{\infty}$, and that its derivative at this fixed point has $\lambda_{z}$ as one of its eigenvalues. We note that the corresponding eigenvector is not $(0, \Omega)$, as equation (5.26) might suggest, but it has a nonzero component in the $y$-direction that has been suppressed in (5.26). The remaining three eigenvalues of $D \Lambda_{H_{*}}\left(0, p_{\infty}\right)$ are obtained by using that $U_{H_{*}}$ is a time and parity preserving symplectic map: they are $\lambda_{\tau}=\vartheta, \lambda_{x}=\mu_{\infty} / \lambda_{z}=-0.706795 \ldots$, and 
$\lambda_{y}=\mu_{\infty} / \lambda_{\tau}=0.1424322345 \ldots$ The corresponding eigenvectors are the direction of the flow at $\left(0, p_{\infty}\right)$, and the vectors $(\Omega, 0)$ and $(0, \omega)$, respectively.

Consider now the RG fixed point $H_{\infty}=H_{*}$. The following result implies Theorem 5.1. It uses the fact that $\lambda_{x}$ is different from the corresponding eigenvalue $-\vartheta^{-1}=-0.618033 \ldots$ of $\mathcal{T}_{\mu}$.

Theorem 5.6. [77] There exists an open neighborhood $V$ of $H_{*}$ in $\mathcal{H}_{\varrho}$ such that if $H_{0}$ belongs to $\mathcal{W}^{s} \cap V$, then the torus $\Gamma_{0}$ defined in Theorem 5.4 is not differentiable.

Proof. By using Lemma 5.2 and the analyticity of $\Lambda_{H_{\infty}}$, we can finds sets $K \subset S$ and $K \subset S^{\prime} \subset K^{\prime}$, all subsets of $D_{\rho^{\prime}}$ whose interiors contain $g=\operatorname{graph}\left(\Gamma_{\infty}\right)$, with $S, S^{\prime}$ open and $K, K^{\prime}$ compact, such that for $H=H_{\infty}$, the scaling map $\Lambda_{H}$ defines an analytic diffeomorphism from $S$ to an open neighborhood of $K^{\prime}$. Since $H \mapsto \Lambda_{H}$ is analytic, this extends to all Hamiltonians $H$ in some open neighborhood of $H_{\infty}$ in $\mathcal{H}_{\rho}$.

Now choose $V$ sufficiently small, such that (our previous results apply and) the set $\bigcup_{n} \operatorname{Range}\left(\Gamma_{n}\right)$ is contained in $K$. This is possible by $(5.22)$, and by the fact that $\mathcal{W}^{s}$ is the graph of an analytic function. Thus, we have

$$
\Gamma_{n}=\Lambda_{n-1}^{-1} \circ \ldots \circ \Lambda_{1}^{-1} \circ \Lambda_{0}^{-1} \circ \Gamma_{0} \circ \mathcal{T}_{1}^{n}
$$

for all $n \geq 0$, where the inverse scalings are defined in an unambiguous way.

In what follows, we lift $\Lambda_{n}$ and $\Gamma_{n}$ from $\mathbb{T}^{2}$ to $\mathbb{R}^{2}$, restrict to $\tau=0$, and use the variables $x, z$. Since $\Lambda_{n}$ is parity preserving, it can be written in the form

$$
\Lambda_{n}(x, z)=\left(f_{n}(x, z) x, \ell_{n}(z)+g_{n}(x, z) x^{2}\right) .
$$

It suffices to consider points in $S^{\prime}$, where we have a bound $|z|<b<\varrho_{z} / 2$. By Proposition 5.5 , there exists a real analytic function $\varphi$ on $[-b, b]$, with non-vanishing derivative, such that $\varphi\left(\ell_{\infty}(z)\right)=\lambda_{z} \varphi(z)$.

Consider now the coordinates $\tilde{z}=\varphi(z)$ and $\tilde{x}=x / \varphi^{\prime}(z)$. Since $U_{H_{\infty}^{\prime}}$ is symplectic, the functions $\ell_{\infty}$ and $f_{\infty}$ in these new coordinates, when restricted to $\tilde{x}=0$, are simply multiplication by $\lambda_{z}$ and $\lambda_{x}$, respectively.

The inverse of $\Lambda_{n}$ is also parity preserving, and thus admits a representation analogous to (5.28). In the coordinates $\tilde{x}$ and $\tilde{z}$, we have

$$
\widetilde{\Lambda}_{n}^{-1}(\tilde{x}, \tilde{z})=\left(\phi_{n}(\tilde{x}, \tilde{z}) \tilde{x}, \psi_{n}(\tilde{x}, \tilde{z})\right)
$$

with $\phi_{n}, \psi_{n}$ analytic in $\widetilde{S}^{\prime}$. Since all derivatives of $\Lambda_{n}$ are bounded on $\widetilde{K}^{\prime}$, uniformly in $n$, we have $\phi_{n}(\tilde{x}, \tilde{z}) \rightarrow \phi_{\infty}(\tilde{x}, \tilde{z})$, uniformly on $\widetilde{S}^{\prime}$. Furthermore, $\phi_{\infty}(\tilde{x}, \tilde{z}) \rightarrow \lambda_{x}^{-1}$, as $\tilde{x} \rightarrow 0$, uniformly in $\tilde{z}$. Thus, if $V$ has been chosen sufficiently small, we can find positive real numbers $\alpha$ and $\beta<1$, such that $\left|\phi_{n}(\tilde{x}, \tilde{y})\right| \leq \beta \vartheta$ for all $n \geq 0$, whenever $|\tilde{x}|<\alpha$. Here, we have used the crucial fact that the eigenvalue $\lambda_{x}$ of $D \Lambda_{\infty}$ at $\Gamma_{\infty}(0)$ is larger in modulus than the corresponding eigenvalue $-\vartheta^{-1}$ of $\mathcal{T}_{1}$.

By equation (5.27), the points $\Gamma_{n}(s, 0)$, expressed in the coordinates $\tilde{x}$ and $\tilde{y}$, are given by

$$
\widetilde{\Gamma}_{n}(s, 0)=\left(\widetilde{\Lambda}_{n-1}^{-1} \circ \ldots \circ \widetilde{\Lambda}_{1}^{-1} \circ \widetilde{\Lambda}_{0}^{-1} \circ \widetilde{\Gamma}_{0}\right)\left(s_{n}, 0\right), \quad s_{n}=\left(-\vartheta^{-1}\right)^{n} s .
$$


Assume now for contradiction that $\Gamma_{0}$ is differentiable at 0 . Then there exists a constant $c>0$ such that for any given $s \in \mathbb{R}$, the angular $(\tilde{x})$ component of $\widetilde{\Gamma}_{0}\left(s_{n}, 0\right)$ is bounded in modulus by $c\left|s_{n}\right|$, for large $n$. Thus, by using the above-mentioned bound on the functions $\phi_{m}$, we find that the angular component of $\widetilde{\Gamma}_{n}(s, 0)$ is bounded in modulus by $c \beta^{n}|s|<\alpha$, if $n$ is sufficiently large.

By using now that $\Gamma_{n} \rightarrow \Gamma_{\infty}$ in $\mathcal{A}_{0}$, and the fact that evaluation is continuous on $\mathcal{A}_{0}$, we conclude that the angular component of $\Gamma_{\infty}(s, 0)$ is zero for all $s \in \mathbb{R}$. Thus, since the curve $s \mapsto s \Omega$ is dense in $\mathbb{T}^{2}$, the angular component of $\Gamma_{\infty}$ is identically zero. But this is impossible, since $\Gamma_{\infty}$ is continuous and homotopic to I. This shows that $\Gamma_{0}$ cannot be differentiable.

QED

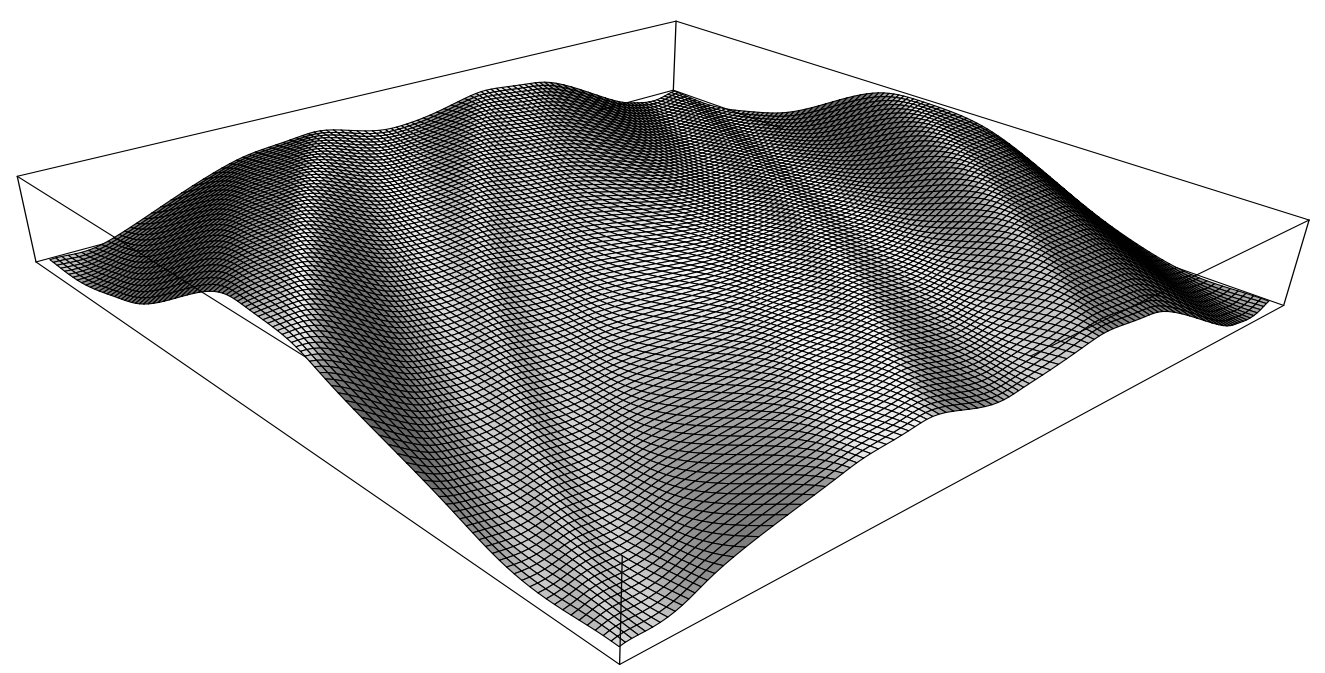

Fig. 8. A critical golden invariant torus

\subsection{Shearless tori}

The construction of invariant tori for Hamiltonians on the stable manifold of an RG fixed point does not involve any nondegeneracy assumptions. Thus, it applies equally well to shearless tori.

Consider again the golden mean case, so the vectors $\omega$ and $\Omega$ are the same as above. We will call an invariant torus shearless if in some coordinates (where the torus is at $p=0$ ),

$$
\dot{q}=c \omega+\kappa(\Omega \cdot p)^{2} \Omega+\mathcal{O}\left(|p|^{3}\right), \quad \dot{p}=\mathcal{O}\left(|p|^{3}\right) .
$$

A simple Hamiltonian with such a torus is $F_{*}(0)$, where

$$
F_{*}(\alpha)(q, p)=\omega \cdot p+\alpha z+\gamma z^{3}, \quad z=\Omega \cdot p .
$$

with $\gamma \in \mathbb{R}$ nonzero. This torus bifurcates, as the parameter $\alpha$ is varied. More specifically, we have $\dot{p}=0$ and $\dot{q}=\omega+\left[\alpha+3 \gamma z^{2}\right] \Omega$, so the Hamiltonian $F_{*}(\alpha)$ has an invariant torus 
at $z=t$ (a constant, not time), and the rotation number of this torus is

$$
\varrho=\frac{\vartheta^{-1}+\left[\alpha+3 \gamma t^{2}\right]}{1-\vartheta^{-1}\left[\alpha+3 \gamma t^{2}\right]} .
$$

Thus, we can distinguish three cases

$\alpha<0: F_{*}(\alpha)$ has two real golden tori (with shear).

$\alpha=0: F_{*}(\alpha)$ has one real golden torus (shearless).

$\alpha>0: F_{*}(\alpha)$ has no real golden torus (but two complex tori).

This type of behavior is observed and expected to persist under small perturbations of the family. For near-integrable area-preserving maps, a theorem confirming this observation was proved in [32], for arbitrary Diophantine rotation numbers, using a KAM type method. The following theorem focuses on golden invariant tori for near-integrable Hamiltonian flows.

Theorem 5.7. [54] If $F$ is real, and sufficiently close to $F_{*}$ in some space of one-parameter families of Hamiltonians, then the following holds. Each $F(\alpha)$ in this family has two golden invariant tori, $\Gamma_{\alpha}^{1}$ and $\Gamma_{\alpha}^{2}$, and no other golden invariant tori nearby. There exists a unique parameter value $\alpha_{F}$ where $\Gamma_{\alpha}^{1}=\Gamma_{\alpha}^{2}$, and the function $F \mapsto \alpha_{F}$ is real analytic. The tori $\Gamma_{\alpha}^{i}$ are real if and only if $\alpha \leq \alpha_{F}$. If the Hamiltonians $F(\alpha)$ are time preserving (a technical assumption to simplify the analysis) then the given torus for $\alpha=\alpha_{F}$ is shearless. The maps $\alpha \mapsto \Gamma_{\alpha}^{i}$ are continuous, and real analytic away from $\alpha_{F}$.

This theorem is proved by using renormalization. We will describe some of the ideas below (with slight modifications, to synchronize the definitions with those used in previous sections). Presumably, the method can be generalized to Diophantine frequencies, using sequences of RG transformations as in [95,81].

The analysis involves two RG transformations with different fixed points and normalizations. One of these transformations is

$$
\mathcal{R}_{0}(H)=H^{\prime} \circ \mathcal{U}_{H^{\prime}}, \quad H^{\prime}=\frac{\vartheta}{\mu} H \circ \mathcal{T}_{\mu}
$$

with $\mu=\mu_{0}$ a fixed positive real number less than $\vartheta^{-1}$. As usual, the canonical transformation $\mathcal{U}_{H^{\prime}}$ is determined in such a way that $\mathbb{I}^{-} \mathcal{R}_{0}(H)=0$. This transformation $\mathcal{R}_{0}$ is considered in a neighborhood of its fixed point $K_{0}(q, p)=\omega \cdot p$, and $\gamma \neq 0$ is fixed (modulo sign) such that $F_{*}(0)$ belongs to this neighborhood. Define

$$
\mathcal{J}_{t} H=H \circ J_{t}, \quad J_{t}(q, p)=\left(q, p+t \Omega^{\prime}\right)
$$

with $\Omega^{\prime}=c \Omega$ normalized in such a way that $\Omega^{\prime} \cdot \Omega=1$. In particular, if $H=F_{*}(\alpha)+h$ then

$$
\left(\mathcal{J}_{t} H\right)(q, p)=\omega \cdot p+\left[\alpha+3 \gamma t^{2}\right] z+3 \gamma t z^{2}+\gamma z^{3}+\left(\mathcal{J}_{t} h\right)(q, p),
$$

modulo a constant term that we can ignore. Then we can choose $\delta>0$ such that the following holds. Consider first the case $h \equiv 0$. Then for $\alpha>\delta$, all orbits for $H$ (in the 
domain $D_{\rho}$ considered) have a rotation number strictly larger than $\vartheta^{-1}$. And for $\alpha<-\delta$, the family $t \mapsto \mathcal{J}_{t} H$ intersects the stable manifold of $\mathcal{R}_{0}$ at $K_{0}$ at exactly two points, and thus $H$ has two golden invariant tori. Furthermore, this intersection is transversal, so the above generalizes to $h \neq 0$ sufficiently close to zero. This argument is used in the proof of Theorem 5.7 to construct the tori $\Gamma_{\alpha}^{i}$ when $\alpha$ is bounded away from zero.

Consider now the Hamiltonians $K_{\gamma}(q, p)=\omega \cdot p+\gamma z^{3}$. With the proper normalization $\mu=\vartheta^{-2}$, these two Hamiltonians are a period 2 for the RG transformation $\mathcal{R}$. For Hamiltonians near $K_{\gamma}$, we choose the RG transformation

$$
\mathcal{R}(H)=H^{\prime} \circ \mathcal{U}_{H^{\prime}}, \quad H^{\prime}=\frac{\vartheta}{\mu} H \circ \mathcal{T}_{\mu} \circ \mathcal{J}_{t},
$$

where $\mu \approx \vartheta^{-2}$ and $t \approx 0$ are determined in such a way that the coefficients of $z^{2}$ and $z^{3}$ in the Fourier-Taylor expansion of $\mathcal{R}(H)-K_{-\gamma}$ are zero. We note that the RG transformations $\mathcal{R}_{0}$ and $\mathcal{R}^{2}$ are hyperbolic near their trivial fixed points, with a one-dimensional unstable direction given by the linear function $(q, p) \mapsto \Omega \cdot p$.

In the case of $\mathcal{R}$, the unstable manifold at $K_{\gamma}$ is in fact given by the family $F_{*}$. Thus, if $F$ is sufficiently close to $F_{*}$, then $F$ intersects the stable manifold of $\mathcal{R}$ at precisely one point. The corresponding parameter value is denoted by $\alpha_{F}$. Using the procedure described in the last section, we can now construct an invariant torus for $F\left(\alpha_{F}\right)$, and if $h=h(q, z)$, then it can be shown that this torus is shearless. One technical complication here is the presence of the translation $\mathcal{J}_{t}$ in the definition (5.37) of $\mathcal{R}$. However, after the first RG step, $t=t(H)$ is of the order of $(\mathbb{I}-\mathbb{E}) H$, where $\mathbb{E}$ denotes averaging over the torus $\mathbb{T}^{2}$. And $(\mathbb{I}-\mathbb{E}) H$ tends to zero very rapidly, so that the translations $\mathcal{J}_{t}$ do not cause any domain problems.

This leaves the problem of constructing invariant tori for $F(\alpha)$ with $\alpha \neq \alpha_{F}$ close to $\alpha_{F}$. This is done by iterating the RG transformation $\mathcal{R}$, until the coefficient of $z$ for $\mathcal{R}^{n}(F(\alpha))$ is larger than $\delta$. Then the RG transformation $\mathcal{R}_{0}$ can be used to construct two golden invariant tori, as described above. This part of the analysis requires good control over the dependence of $\mathcal{R}(F(\alpha))$ on the parameter $\alpha$. Thus, it is convenient to introduce an RG transformation for families $\alpha \mapsto F(\alpha)$. In what follows, we identify the parameter space $\mathbb{C}$ with the one-dimensional expanding subspace of $D \mathcal{R}\left(K_{0}\right)$. Denoting by $\mathbb{P}$ the canonical projection onto this subspace, we define

$$
Y_{F}(\alpha)=\mathbb{P} \mathcal{R}(F(\alpha)) .
$$

In particular, $Y_{F_{*}}(\alpha)=-\vartheta^{2} \alpha$, which reflects the fact that $F_{*}$ is the unstable manifold of $\mathcal{R}$ at $K_{0}$, and that the expanding eigenvalue is $-\vartheta^{2}$. The map $Y_{F}$ is now used to re-parametrize the family $F$ after renormalization. More specifically, we set

$$
\mathfrak{R}(F)=\mathcal{R} \circ F \circ Y_{F}^{-1} .
$$

By construction, $F_{*}$ is a fixed point of $\mathfrak{R}$, and it is straightforward to check that all eigenvalues of $D \mathfrak{R}\left(F_{*}\right)$ are of modulus less than one. Thus, we expect that $\mathfrak{R}^{n}(F) \rightarrow F_{*}$ for large $n$, This property, which was proved in [1], makes it possible to get the necessary control over $\mathcal{R}^{n}(F(\alpha))$ for $\alpha \neq \alpha_{F}$ very close to $\alpha_{F}$, and for $n$ correspondingly large. 
This procedure, known as the graph transform method, is a common method for constructing the (un)stable manifold of a map $\mathcal{R}$; see e.g. [64]. We will describe a generalization (to sequences of maps) of this method in Subsection 7.4.

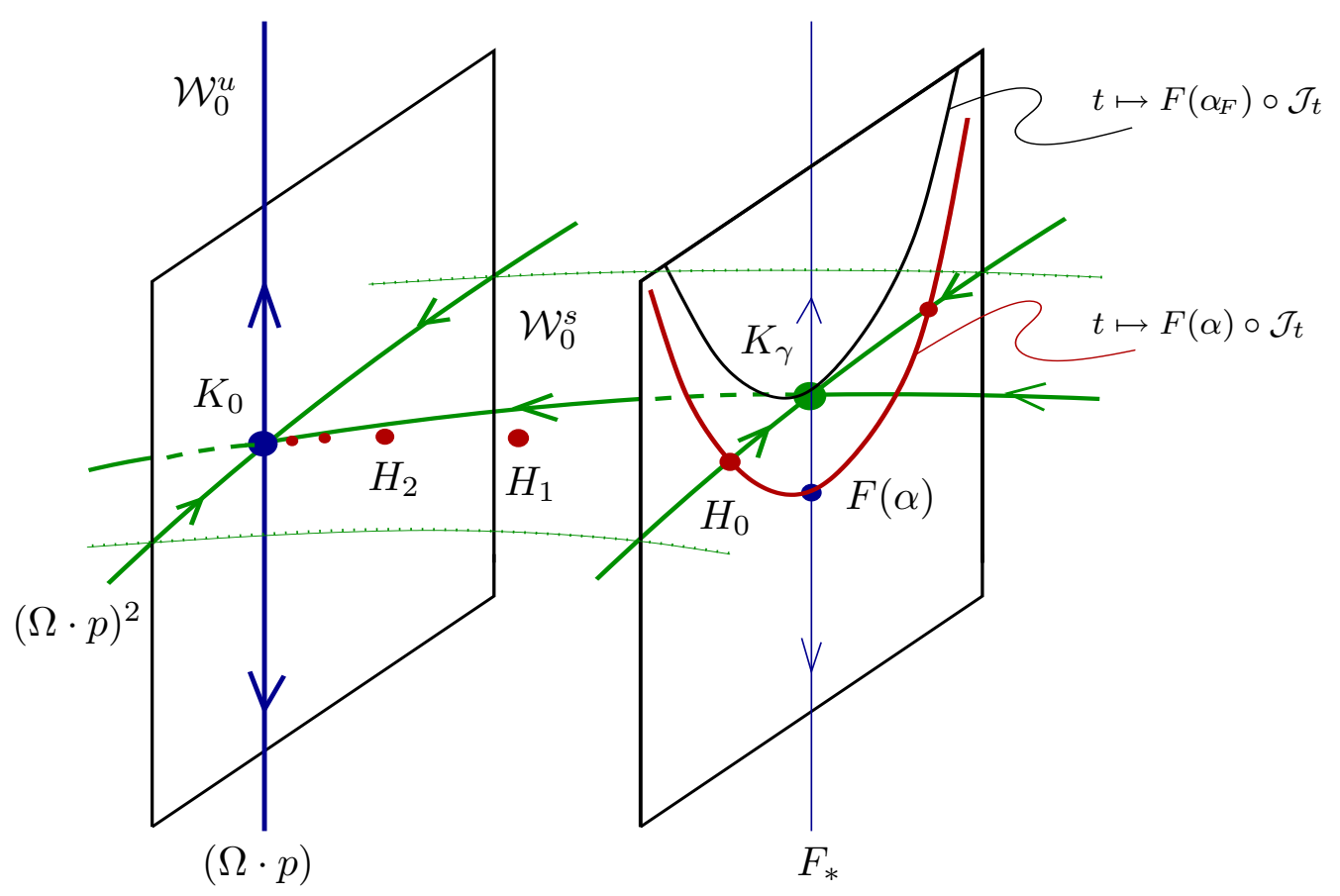

Fig. 9. Renormalization of families with shearless invariant tori

\section{Scaling}

According to the general RG picture, the properties of a hyperbolic RG fixed point $H_{\infty}$ with an $m$-dimensional unstable manifold describes universal behavior for $m$-parameter families $\beta \mapsto H^{\beta}$. The universal constants are eigenvalues of either the linearized RG transformation $D \mathcal{R}\left(H_{\infty}\right)$ or the linearized scaling map $D \Lambda_{H_{\infty}}\left(q_{\infty}, p_{\infty}\right)$. The latter describe self-similarity properties of the orbit structure for Hamiltonians near the critical surface (the stable manifold of $\mathcal{R}$ at $H_{\infty}$ ). The reason is pretty clear: If $G$ is an orbit for $\mathcal{R}(H)$, with rotation vector $w$, then $\Lambda_{H}(G)$ is an orbit for $H$, with rotation vector parallel to $T w$. This fact was used e.g. in the analysis of invariant tori. Here, we discuss properties that relate to eigenvalues of the linearized RG transformation, and more specifically, the rate at which periodic orbits accumulate at an invariant torus.

\subsection{Spectrum of the linearized RG transformation}

If $H$ is a purely resonant Hamiltonian, so that $\mathcal{U}_{H^{\prime}}$ is the identity, then $D \mathcal{R}(H)$ can be obtained explicitly, since it suffices for this purpose to eliminate nonresonant modes to first order in the perturbation. In this case, the derivative of $\mathcal{N}: H \mapsto \mathcal{U}_{H}$ is given by the 
equation

$$
D \mathcal{N}(H)=\mathbb{I}^{+}-\mathbb{I}^{+} \widehat{H}\left(\mathbb{I}^{-} \widehat{H} \mathbb{I}^{-}\right)^{-1} \mathbb{I}^{-}, \quad \mathbb{I}^{-} H=0 .
$$

This simplifies to $D \mathcal{N}(H)=\mathbb{I}^{+}$, if $H(q, p)$ does not depend on the angle variable $q$. In order to simplify things further, consider now $\mathcal{R}$ with fixed scaling parameters $\eta=\vartheta_{1}^{-1}$ and $0<\mu<\vartheta_{d}$. Here, and in what follows, $\vartheta_{k}$ denotes the $k$-th largest eigenvalue (in modulus) of the matrix $T$, and $\omega_{k}$ denotes the corresponding eigenvector. In particular, $\vartheta_{1}=\vartheta$ and $\omega_{1}=\omega$. The Hamiltonian $K_{0}(q, p)=\omega \cdot p$ is a fixed point of this transformation $\mathcal{R}$. If we write $f(q, p)=\sum_{\nu} f_{\nu}(p) e^{i \nu \cdot q}$, then

$$
\left(D \mathcal{R}\left(K_{0}\right) f\right)(q, p)=\sum_{\nu} \frac{\vartheta_{1}}{\mu} f_{\nu}\left(\mu\left(T^{*}\right)^{-1} p\right) \exp (i \nu \cdot(T q)) .
$$

Assume now that $f$ is an analytic eigenfunction of $D \mathcal{R}\left(K_{0}\right)$, with eigenvalue $\lambda \neq 0$. Then we obtain

$$
f_{T^{n} \nu}=\left(\frac{\vartheta_{1}}{\lambda \mu}\right)^{n} f_{\nu}\left(\mu^{n}\left(T^{*}\right)^{-n} \cdot\right), \quad \nu \in \mathbb{Z}^{d},
$$

first for $n=1$, and then for any $n \in \mathbb{N}$ by iteration. Consider $\nu \neq 0$. Then $T^{n} \nu \sim \vartheta^{n}$ for large $n$, so by analyticity, the left hand side of (6.3) is bounded in modulus by $\exp \left(-c \vartheta^{n}\right)$, for some $c>0$. But the right hand side can tend to zero no faster than exponentially, unless $f_{\nu}=0$. This shows that the eigenvectors of $D \mathcal{R}\left(K_{0}\right)$ are functions of $p$ only. Finding these is easy: On the (invariant) subspace of functions $f(q, p)=f_{0}(p)$, the homogeneous polynomials $(q, p) \mapsto\left(\omega_{k} \cdot p\right)^{m}$ and their products are a basis of eigenvectors. In what follows, we will always choose

$$
0<\mu<\left|\vartheta_{1}^{-1} \vartheta_{d}^{2}\right|
$$

Then $D \mathcal{R}\left(K_{0}\right)$ has exactly $d$ eigenvalues of modulus $>1$. One of them is $\lambda_{0}=\vartheta / \mu$, associated with constant Hamiltonians, and the other $d-1$ eigenvalue-eigenvector pairs are

$$
\lambda_{k}=\vartheta_{1} / \vartheta_{k}, \quad f_{k}(q, p)=\omega_{k} \cdot p, \quad k=2, \ldots, d .
$$

Since the value of $\mu$ has been fixed, $D \mathcal{R}\left(K_{0}\right)$ also has an eigenvalue 1 , with eigenvector $f_{1}(q, p)=\omega \cdot p$. But we can (and will) reduce this eigenvalue to 0 by choosing a scaling $\mu=\mu(H)$ in such a way that the torus average of $\omega \cdot \nabla_{p} H$ is a fixed positive constant. Then all eigenvalues of $D \mathcal{R}\left(K_{0}\right)$ other than the ones listed in (6.5) are of modulus $<1$.

It should be noted that the expanding eigenvalues (including $\lambda_{0}$ ) could be mapped to 0 as well, by including a translation $(q, p) \mapsto(q, p+v)$ in the definition of $\mathcal{R}$, with $v=v(H)$ chosen appropriately. In this sense, $D \mathcal{R}\left(K_{0}\right)$ has no "relevant" expanding eigenvalues. By contrast, the linearized RG transformation at the critical fixed point $H_{\infty}$ described in Theorem 4.1 is believed to have an expanding eigenvalue $\delta=1.6279 \ldots$ that is not related to coordinate changes.

\subsection{Accumulation of periodic orbits}

The expanding eigenvalues of the linearized RG transformation describe asymptotic relations between different members of the family $\beta \mapsto H^{\beta}$, as $\beta$ approaches the value $\beta_{\infty}$ 
where the family intersects the stable manifold $\mathcal{W}^{s}$ of the given $\mathrm{RG}$ fixed point $H_{\infty}$. To give an example, let $\mathcal{P}$ be a collection of properties that can be assigned to closed orbits, such that if $\mathcal{R}(H)$ has an orbit of type $\mathcal{P}$, then the corresponding orbit of $H$ is also of type $\mathcal{P}$. Given some fixed $w \in \mathbb{R} \mathbb{Z}^{d}$ close to $\omega$, consider the set of Hamiltonians in the domain of $\mathcal{R}$ that have a closed orbit of type $\mathcal{P}$ with rotation vector parallel to $w$. Assume that this set contains a codimension $m$ manifold $\Sigma_{0}$ that is transversal to the local unstable manifold $\mathcal{W}^{u}$ of $\mathcal{R}$.

Now consider the sets $\Sigma_{n}=\mathcal{R}^{-n}\left(\Sigma_{0}\right)$. They consist of Hamiltonians that have closed orbits of type $\mathcal{P}$ with rotation vectors parallel to $T^{n} w$. By the $\lambda$-lemma [113], these sets are codimension $m$ manifolds that accumulate at $\mathcal{W}^{s}$ at an asymptotic rate given by the largest eigenvalue $\delta$ of $D \mathcal{R}\left(H_{\infty}\right)$. Or more precisely, if $\beta \mapsto H^{\beta}$ is a $m$-parameter family that intersects $\mathcal{W}^{s}$ transversally, then for large $n$, this family intersects $\Sigma_{n}$ at exactly one point, say $H^{\beta_{n}}$, and $\left(\beta_{n+1}-\beta_{n}\right) /\left(\beta_{n}-\beta_{n-1}\right) \rightarrow \delta^{-1}$. In particular, if one of the properties characterizing $\Sigma_{0}$ is marginal stability, then we obtain the observed accumulation of bifurcation points described earlier (where $m=1$, after elimination of the unstable directions related to $p$-translations). A stronger property that is expected to hold is the convergence of $\mathcal{R}^{k}\left(H^{\beta_{n+k}}\right)$ to the intersection point of $\Sigma_{n}$ with $\mathcal{W}^{u}$. This describes a joint scaling in parameter space and phase space.

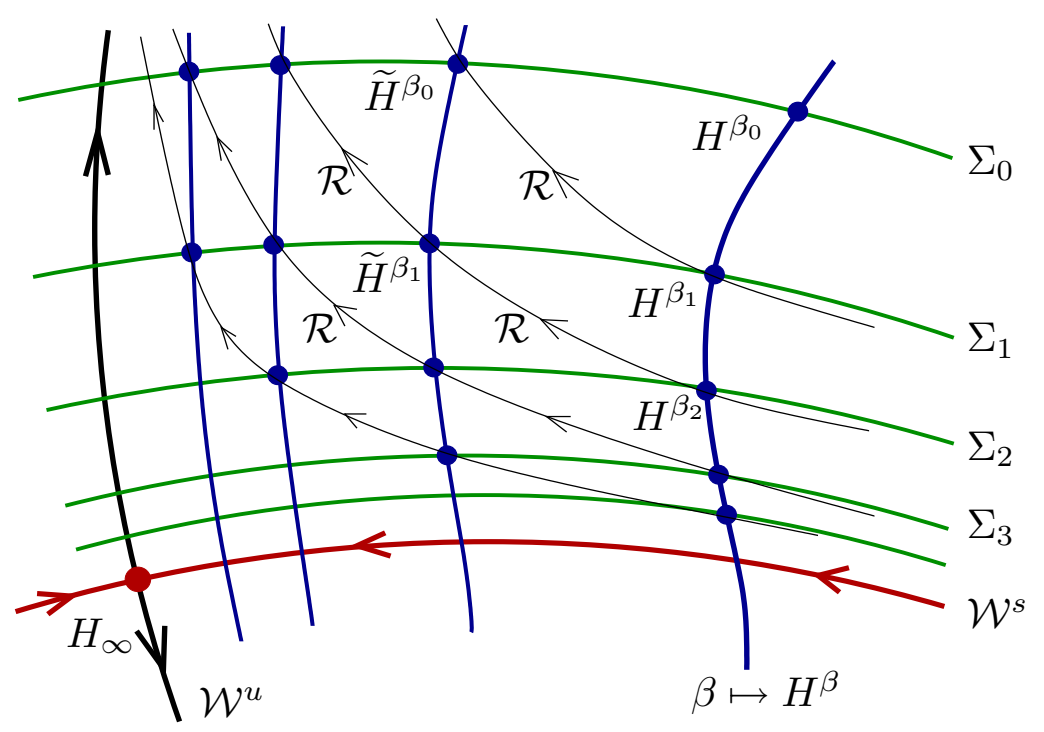

Fig. 10. Expected RG picture explaining universality

Our goal here is to prove that such a picture is correct near the trivial fixed point $K_{0}$. The RG transformation $\mathcal{R}$ is as described in Theorem 2.2, and we use the same space $\mathcal{A}_{\rho}$ of analytic Hamiltonians as in Subsection 2.2. In this analysis, we will keep track of constant terms in Hamiltonians, so the unstable manifold $\mathcal{W}^{u}$ is of dimension $d$. As a result, the invariant torus $\Gamma_{H}$ for a Hamiltonian $H$ on the stable manifold $\mathcal{W}^{s}$ is not only centered 
at zero, but also lies on the surface of zero energy, $H \circ \Gamma_{H}=0$. The families $\beta \mapsto H^{\beta}$ considered are generated by a single (isoenergetically nondegenerate) Hamiltonian $H$ via $p$-translations,

$$
H^{\beta}=H \circ J_{\beta}, \quad J_{\beta}(q, p)=(q, p+\beta),
$$

with $\beta \in \mathbb{R}^{d}$. Thus, the periodic orbits described above are all orbits of the same Hamiltonian $H$. The rate of accumulation of the parameter values $\beta_{n}$ describes the rate of accumulation of periodic orbits at the invariant torus.

In order to ensure that the family $\beta \mapsto H^{\beta}$ intersects the stable manifold $\mathcal{W}^{s}$ transversally, we will assume that $H$ lies near an isoenergetically nondegenerate integrable Hamiltonian $K$ of the form (5.8). Still, considering such near-integrable Hamiltonians poses a problem. If $K$ has a closed orbit $\gamma$ with rotation vector $w$, then it has infinitely many of them: the entire torus $p=p_{w}$ containing $\gamma$ consists of such orbits. But a Hamiltonian $H$ close to $K$ has in general only finitely many $w$-orbits (closed orbits with rotation vector parallel to $w$ ). The $q$-translates of $H$ are equally close to $K$, but their $w$-orbits vary by an amount of order one. Thus, there is no continuous map near $K$ (or near any integrable Hamiltonian) that would associate with a Hamiltonian one of its $w$-orbits. That is, unless we prevent the ( $q$-translation) symmetry of $K$ from acting on our space of Hamiltonians.

We will do this by restricting the analysis to the subspace $\mathcal{B}_{\rho}$ of Hamiltonians $H(q, p)$ that are even functions of $q$. Then it is possible to find closed orbits of the form

$$
\gamma(t)=\left(t w+Q(t / \tau), P_{0}+P(t / \tau)\right),
$$

where $Q$ is an odd and $P$ an even $2 \pi$-periodic function with average zero. The rotation vector $w$ belongs to $\mathbb{R} \mathbb{Z}^{d}$, and $\tau=\tau(w)$ is defined as the smallest positive real number $t$ such that $t w$ belongs to $\mathbb{Z}^{d}$. We will refer to such an orbit as a symmetric $w$-orbit for $H$.

To be more precise, we will focus on orbits on the energy surface $H=0$. Then we cannot prescribe the frequencies $w_{j}$, but only the winding numbers $w_{j} / w_{d}$.

Define $h_{w}(q, p)=w \cdot p$ and

$$
A(\gamma)=\frac{1}{2 \pi} \oint_{\gamma} \sum_{j=1}^{d} p_{j} d q_{j} .
$$

Theorem 6.1. [1] Let $R>\rho$, and let $K \in \mathcal{B}_{r}$ be a Hamiltonian of the form (5.8), with $M$ as described after (5.8). If $K$ sufficiently close to $h_{\omega}$, and $w \in \mathbb{R}^{d}$ sufficiently close to $\omega$, then there exists an open neighborhood $B$ of $K$ in $\mathcal{B}_{R}$, and a positive integer $N$, such that for every Hamiltonian $H \in B$, and every $n \geq N$, some nonzero constant multiple of $H$ has a symmetric orbit $\gamma_{n}$ with frequency vector $w_{n}=\left(\vartheta^{-1} T\right)^{n} w$, lying on the energy surface $H^{-1}(0)$, and having $A\left(\gamma_{n}\right)=0$. The sequence of orbit $\gamma_{n}$ satisfy

$$
-\frac{1}{n} \ln \left|\gamma_{n}(0)-\Gamma(0)\right|=\ln \left|\lambda_{2}\right|+\mathcal{O}\left(\frac{1}{n}\right),
$$

where $\Gamma$ is the invariant torus for $H$, obtained via translation from the torus $\Gamma_{H^{\prime}}$ for the Hamiltonian $H^{\prime}=H^{\beta_{\infty}}$. 
The proof of this theorem will be described in the next two subsections. The estimate (6.9) is obtained from an analogous estimate

$$
-\frac{1}{n} \ln \left|\beta_{n}-\beta^{\prime}\right|=\ln \left|\lambda_{2}\right|+\mathcal{O}\left(\frac{1}{n}\right)
$$

for the parameter values $\beta_{n}$ defining the intersection of the family $\beta \mapsto H^{\beta}$ with the manifolds $\Sigma_{n}$.

\subsection{Choice of the manifold $\Sigma_{0}$}

We assume that $w \in \mathbb{R}^{d}$ is chosen sufficiently close to $\omega$, such that $h_{w}$ belongs to the domain of $\mathcal{R}$, and such that $v \cdot w=1$, where $v$ is the expanding eigenvector of $T^{*}$.

As part of the construction of $\sigma_{0}$, we consider $d+1$ parameter families of Hamiltonians. One of the parameters, $\xi$, is used to determine which multiple of $H$ lies on $\sigma_{0}$, and the other $d$ parameters, $(u, E)$, can be related to $p$-translations. Given $\xi \in \mathbb{C}$, and $u \in \mathbb{C}^{d}$ satisfying $u \cdot v=0$, define $x=(\xi-1) w+\xi u$. Let $H=h_{w}+h$. Our goal is to determine the values of $\xi, u$, and $E$, such that the Hamiltonian

$$
F=\xi\left(H+h_{u}\right)+E=h_{w}+\xi h+h_{x}+E
$$

has a symmetric $w$-orbit $\gamma$ with the desired properties. Besides the two properties $F \circ \gamma=0$ and $A(\gamma)=0$, that are invariant under renormalization, we also impose that $P_{0}=k v$, for some $k \in \mathbb{C}$. Roughly speaking, the conditions $F \circ \gamma=0$ and $A(\gamma)=0$ determine $E$ and $k$, respectively, while $P_{0}=k v$ determines the parameter $u$. In what follows, we will treat $x=(\xi-1) w+\xi u$ as an independent parameter, and treat $(\xi, u)$ as a function of $x$.

We start by solving the orbit equation $\dot{\gamma}=(\mathbb{J} \nabla F) \circ \gamma$. The $q$-component of this equation can be rewritten as

$$
\tau^{-1} D Q=\left(\nabla_{2}\left(\xi h+h_{x}\right)\right) \circ \gamma=\xi\left(\nabla_{2} h\right) \circ \gamma+x .
$$

The torus average (denoted by $\mathbb{E}$ ) of the right hand side has to vanish, so

$$
x=\widetilde{x}, \quad \widetilde{x}=-\xi \mathbb{E}\left[\left(\nabla_{2} h\right) \circ \gamma\right] .
$$

The corresponding equation for remaining zero-average part is

$$
Q=\widetilde{Q}, \quad \widetilde{Q}=\tau \xi D^{-1}(\mathrm{I}-\mathbb{E})\left[\left(\nabla_{2} h\right) \circ \gamma\right] .
$$

Similarly, the $p$-component of the orbit equation can be rewritten as $\tau^{-1} D P=-\xi\left(\nabla_{1} h\right) \circ \gamma$, or, since both sides of this equation are odd functions of $q$, as

$$
P=\widetilde{P}, \quad \widetilde{P}=\tau \xi D^{-1}\left[\left(\nabla_{1} h\right) \circ \gamma\right] .
$$

Next, consider the condition $A(\gamma)=0$. By using equation (6.12), we have

$$
\begin{aligned}
A(\gamma) & =\frac{1}{2 \pi} \oint_{\gamma} p \cdot d q=\frac{1}{2 \pi} \int_{0}^{2 \pi \tau}(k v+P) \cdot\left(w+\tau^{-1} D Q\right) d t \\
& =k \tau+\frac{1}{2 \pi \tau} \int_{0}^{2 \pi \tau} P \cdot D Q d t=k \tau+\frac{\xi}{2 \pi} \int_{0}^{2 \pi \tau} P \cdot\left(\nabla_{2} h\right) \circ \gamma d t
\end{aligned}
$$


Thus, the condition $A(\gamma)=0$ is equivalent to

$$
k=\widetilde{k}, \quad \widetilde{k}=-\frac{\xi}{2 \pi \tau} \int_{0}^{2 \pi \tau} P \cdot\left(\nabla_{2} h\right) \circ \gamma d t .
$$

Finally, consider the condition $F \circ \gamma=0$. By using that

$$
\begin{aligned}
F \circ \gamma & =\frac{1}{2 \pi \tau} \int_{0}^{2 \pi \tau} F \circ \gamma d t=E+\frac{\xi}{2 \pi \tau} \int_{0}^{2 \pi \tau}\left[h_{w}+h+h_{u}\right] \circ \gamma d t \\
& =E+k+\frac{\xi}{2 \pi \tau} \int_{0}^{2 \pi \tau} h \circ \gamma d t
\end{aligned}
$$

we obtain the following equation:

$$
E=\widetilde{E}, \quad \widetilde{E}=\frac{\xi}{2 \pi \tau} \int_{0}^{2 \pi \tau}\left(h-P \cdot \nabla_{2} h\right) \circ \gamma d t
$$

The equations (6.13) ... (6.17) define a fixed point problem for the map $\psi$ that takes $(x, E, k, Q, P)$ to $(\widetilde{x}, \widetilde{E}, \widetilde{k}, \widetilde{Q}, \widetilde{P})$. For the components $Q$ and $P$, consider the space of $\mathbb{C}^{d}$-valued analytic functions on the strip $|\operatorname{Im} z|<r$, equipped with the sup-norm, where $r=\rho /(3 \tau)$. And on the space of quintuples $q=(x, E, k, Q, P)$, consider as norm the largest component norm. Then it is easy to see that $\psi$ is a contraction near $q=0$, with $\psi(q)=\mathcal{O}\left(\|h\|_{\rho}\right)$. As a result we find that for each Hamiltonian $H$ in some open neighborhood $B(w)$ of $h_{w}$ in $\mathcal{B}_{\rho}$, the equation $F(q)=q$ has a unique solution near $q=0$. This solution yields a set of parameters $(\xi, u, E)$, and a $w$-orbit $\gamma$ for the corresponding Hamiltonian $F$, satisfying

$$
|\xi-1|,|u|,|E|,|k|,\|Q\|_{r},\|P\|_{r} \leq C\left\|H-h_{w}\right\|_{\rho}
$$

for some constant $C>0$. Furthermore, all these quantities depend analytically on $H$.

Now define

$$
\phi(H)=h_{u}+E / \xi,
$$

so that $\xi(H+\phi(H))=F$. Consider this function $\phi$ on $X \cap B(w)$, where $X$ is the subspace of $\mathcal{B}_{\rho}$ consisting of functions of the form $c h_{w}+f$, with $f$ having zero Fourier-Taylor coefficients $f_{\nu, \alpha}$ whenever $\nu=0$ and $|\alpha| \leq 1$. The range of $\phi$ is contained in the $d$ dimensional expanding subspace $W^{u}$ of $D \mathcal{R}\left(h_{\omega}\right)$. Clearly, $X$ is transversal to $W^{u}$. Thus, the graph of $\phi$, which we shall denote by $\Sigma(w)$, is transversal to the unstable manifold $\mathcal{W}^{u}$ of $\mathcal{R}$ at the fixed point $h_{\omega}$.

Denote by $\Sigma^{\prime}(w)$ the set of Hamiltonians $H$ in the domain of $\mathcal{R}$, with the property that a constant multiple of $H$ has a symmetric $w$-orbit $\gamma$ on the energy surface $H=0$, satisfying $A(\gamma)=0$. By construction, $\Sigma(w)$ is a subset of $\Sigma^{\prime}(w)$. 


\subsection{The manifolds $\Sigma_{n}$ and orbits $\gamma_{n}$}

Now consider the sets $\Sigma_{n}(w)=\mathcal{R}^{-n}(\Sigma(w))$. By the $\lambda$-lemma [113], these sets are codimension $d$ manifolds that are transversal to $\mathcal{W}^{u}$, and that accumulate at $\mathcal{W}^{s}$ as indicated by equation (6.10). Here, the assumption is that the given family $F$ is transversal to the stable manifold $\mathcal{W}^{s}$, and close to the unstable manifold. For our families $\beta \mapsto H^{\beta}$, the transversality condition is guaranteed by the assumptions on $K, R$, and $B$ in Theorem 6.1. But in order to get sufficiently close to the family $F_{*}$ parametrizing the unstable manifold, we first have to renormalize $F: \beta \mapsto H^{\beta}$ a few times.

An appropriate $\mathrm{RG}$ transformation $\mathfrak{R}$ for families is given by

$$
\mathfrak{R}(F)=\mathcal{R} \circ F \circ Y_{F}^{-1}, \quad Y_{F}(\beta)=\mathbb{P} \mathcal{R}(F(\beta)),
$$

as described in Subsection 5.5; see also Subsection 7.4. Here, $\mathbb{P}$ denotes the canonical projection onto the $d$-dimensional unstable subspace $W^{u}$ of $D \mathcal{R}\left(h_{\omega}\right)$, which we identify with the parameter space $\mathbb{C}^{d}$. An explicit computation yields

$$
\left(D \Re\left(F_{*}\right) f\right)(\beta)=(\mathbb{I}-\mathbb{P}) D \mathcal{R}\left(F_{*}(\widetilde{\beta})\right) f(\widetilde{\beta}), \quad \widetilde{\beta}=Y_{F_{*}}(\beta) .
$$

This shows e.g. that, the largest eigenvalue (in modulus) of $D \mathfrak{R}\left(F_{*}\right)$ is the largest contracting eigenvalue of $D \mathcal{R}\left(h_{\omega}\right)$, which is $\mu \vartheta_{1} \vartheta_{d}^{-2}$. In what follows, we assume that the scaling $\mu$ has been chosen in such a way that

$$
\left|\mu \vartheta_{1} \vartheta_{d}^{-2}\right|<\epsilon<\left|\lambda_{2}^{-1}\right|
$$

Consider now $F_{k}=\mathfrak{R}^{k}(F)$, with $F(\beta)=H^{\beta}$ and $H$ satisfying the assumptions of Theorem 6.1. Since $F_{k} \rightarrow F_{*}$ as $k \rightarrow \infty$, the intersection parameters for $F_{k}$ satisfy (6.10), if $k$ is sufficiently large. But then the same holds for $F$ as well.

The transformation $\mathfrak{R}$ can also be used to obtain the necessary bounds $(6.10)$ on the parameter values $\beta_{n}$. More specifically, since $F_{n} \rightarrow F_{*}$, we have

$$
\beta_{n}=Y_{F_{0}}^{-1} \circ \ldots \circ Y_{F_{n-1}}\left(b_{n}\right), \quad F_{n}\left(b_{n}\right) \in \Sigma(w),
$$

for some (unique) parameter value $b_{n}$, if $n$ is sufficiently large. Notice that $Y_{F_{*}}$ is simply the restriction of $D \mathcal{R}\left(h_{\omega}\right)$ to the unstable subspace $Y$. Thus, the largest eigenvalue (in modulus) of $Y_{F_{*}}^{-1}$ is $\lambda_{2}^{-1}$, and (6.10) obviously holds for the family $F_{*}$. In order to prove the same for other families like $\beta \mapsto H^{\beta}$, one uses that $F_{n}-F_{*}$ and $Y_{F_{n}}-Y_{F_{*}}$ and $b_{n}-b_{\infty}$ are of the order $\mathcal{O}\left(\epsilon^{n}\right)$, which is small compared to $\left|\lambda_{2}\right|^{-n}$.

Our next goal is to construct the orbits $\gamma_{n}$ described in Theorem 6.1, and to estimate the values $\gamma_{n}(0)$. Given any Hamiltonian in the domain of $\mathcal{R}^{n}$, the renormalized Hamiltonian $H_{n}=\mathcal{R}^{n}(H)$ is a constant multiple of $H \circ \widetilde{\mathcal{V}}_{n}(H)$, where

$$
\left.\widetilde{\mathcal{V}}_{n}(H)=\mathcal{V}_{0} H\right) \circ \ldots \circ \mathcal{V}_{n-1}(H), \quad \mathcal{V}_{k}(H)=\mathcal{T}_{\mu}^{k} \circ \mathcal{U}_{H_{k}} \circ \mathcal{T}_{\mu}^{-k}
$$

If $H$ is sufficiently close to the integrable Hamiltonian $K$, then $H_{n}^{\beta_{n}}$ lies on the manifold $\Sigma(w)$, and thus some nonzero constant multiple of $H_{n}^{\beta_{n}}$ has a symmetric $w$-orbit $g_{n}$. Modulo domain questions, this yields a symmetric $w_{n}$-orbit

$$
G_{n}=\widetilde{\mathcal{V}}_{n}\left(H^{\beta_{n}}\right) \circ \mathcal{T}_{\mu}^{n} \circ g_{n} \circ \Theta^{-n}, \quad \Theta(t)=\vartheta t,
$$


for a constant multiple of $H_{\beta_{n}}$. By construction, the size of the domain of the transformations $\widetilde{\mathcal{V}}_{n}(H)$ decreases exponentially with $n$, but at a rate that is independent of $\mu$. By comparison, the nonlinear part of $g_{n}$ is bounded by a constant times the norm of $H_{n}^{\beta_{n}}-h_{w}$, as was shown in (6.18), and this norm decreases like $\mathcal{O}\left(\epsilon^{n}\right)$. Thus, if $\mu$ is chosen sufficiently small, then the maps in equation (6.25) can be composed as indicated, yielding the desired orbits $G_{n}$.

By using that $\gamma_{n}=J_{\beta_{n}} G_{n}$ and $\Gamma=J_{\beta_{\infty}} \Gamma_{H^{\prime}}$, we have

$$
\gamma_{n}(0)-\Gamma(0)=\left(\beta_{n}-\beta_{\infty}\right)+\left[G_{n}(0)-\Gamma_{H^{\prime}}(0)\right] .
$$

Thus, it suffices to show that the term [...] is small (in modulus) compared to $\beta_{n}-\beta_{\infty}$. To this end, write

$$
\begin{aligned}
\left|G_{n}(0)-\Gamma_{H^{\prime}}(0)\right| & \leq\left|\widetilde{\mathcal{V}}_{n}\left(H^{\beta_{n}}\right)\left(T_{\mu}^{n}\left(g_{n}(0)\right)\right)-\widetilde{\mathcal{V}}_{n}\left(H^{\beta_{n}}\right)(0)\right| \\
& +\left|\widetilde{\mathcal{V}}_{n}\left(H^{\beta_{n}}\right)(0)-\widetilde{\mathcal{V}}_{n}\left(H^{\prime}\right)(0)\right|+\left|\widetilde{\mathcal{V}}_{n}\left(H^{\prime}\right)(0)-\Gamma_{H^{\prime}}(0)\right|
\end{aligned}
$$

Since $g_{n}(0)=\mathcal{O}\left(\varepsilon^{n}\right)$, as mentioned earlier, the first term on the right hand side of (6.27) is bounded by $c(b \epsilon)^{n}$, for some constants $b, c>0$. The third term admits a similar bound, since it describes the convergence of $\widetilde{\mathcal{V}}_{n}\left(H^{\prime}\right) \rightarrow \Gamma_{H^{\prime}}$, for $H^{\prime} \in \mathcal{W}^{s}$, which is governed by the largest contracting eigenvalue of $D \mathcal{R}\left(h_{\omega}\right)$. In order to estimate the second term, we can use the analyticity of $f \mapsto \widetilde{\mathcal{V}}_{n}(f)$, to obtain a bound of the form

$$
\left|\widetilde{\mathcal{V}}_{n}\left(H_{\beta_{n}}\right)(0)-\widetilde{\mathcal{V}}_{n}\left(H^{\prime}\right)(0)\right| \leq C\left|\lambda_{2}\right|^{-n}\|H-K\|_{\rho} .
$$

The details leading to these estimates can be found in [1]. Putting it all together, we find that (6.9) holds for large $n$, provided that $\mu$ is chosen sufficiently small and $H$ sufficiently close to $K$. It should be noted that the smallness condition on $\mu$ only depends on the matrix $T$.

\section{Sequences of RG transformations}

So far, we have considered only "self-similar" rotation vectors $\omega \in \mathbb{R}^{d}$ that are eigenvectors of some integer matrix $T$ with determinant \pm 1 . Extending renormalization to more general rotation vectors involves using sequences $\left\{T_{n}\right\}$ of such matrices, coming from a continued fraction expansion for $\omega$; see also references $[71,72,83]$. At this point (having kept track of how the bounds on $\mathcal{R}$ depend on the matrix $T$ ), the main ingredients in this extension are a multidimensional continued fraction expansion, with bounds on the matrices $T_{n}$, and a stable manifold theorem that can be applied to sequences of RG transformations. Both will be discussed in this section.

\subsection{Diophantine and Brjuno numbers}

In the case $d=2$, a self-similar rotation vector is a vectors $\omega=\left(u_{0}, v_{0}\right)$ whose winding number $\alpha_{0}=v_{0} / u_{0}$ has a continued fraction expansion (for $n=0$ )

$$
\alpha_{n}=\left[a_{n} ; a_{n+1}, a_{n+1}, \ldots\right]=a_{n}+\frac{1}{a_{n+1}+\frac{1}{a_{n+2}+\ldots}}
$$


that is periodic. Here, we have assumed that $\alpha_{0}>0$. The positive integers $a_{n}$ in the continued fractions expansion of an irrational $\alpha_{0}>0$ are obtained inductively,

$$
a_{n}=\left\lfloor\alpha_{n}\right\rfloor, \quad \alpha_{n+1}=\frac{1}{\alpha_{n}-a_{n}}, \quad n=0,1,2, \ldots
$$

where $\alpha \mapsto\lfloor\alpha\rfloor$ is the floor (integer part) function on $\mathbb{R}$. Alternatively, the fractional parts $x_{n}=\alpha_{n}-a_{n}$ can be obtained by iterating $x_{n+1}=G\left(x_{n}\right)$, where $G$ is the Gauss map taking $x$ to the fractional part of $x^{-1}$. The relation (7.2) between $\alpha_{n}$ and $\alpha_{n+1}$ can also be written in the form

$$
\left[\begin{array}{c}
1 \\
\alpha_{n+1}
\end{array}\right]=\alpha_{n+1} T_{n}^{-1}\left[\begin{array}{c}
1 \\
\alpha_{n}
\end{array}\right], \quad T_{n}=\left[\begin{array}{cc}
0 & 1 \\
1 & a_{n}
\end{array}\right] .
$$

Thus, if we start e.g. with $\omega_{0}=\left(1, \alpha_{0}\right)$ and define $\omega_{n+1}=\alpha_{n+1} T_{n}^{-1} \omega_{n}$ for $n=0,1, \ldots$, then $\omega_{n}=\left(1, \alpha_{n}\right)$.

A renormalization procedures for Hamiltonians on $\mathbb{T}^{2} \times \mathbb{R}^{2}$, that "zooms in" on orbits with rotation number $\alpha_{0}$, involves a sequence of RG transformations $\mathcal{R}_{n}$ associated with the sequence of matrices $T_{n}$,

$$
\mathcal{R}_{n}(H)=H^{\prime} \circ \mathcal{U}_{H^{\prime}}, \quad H^{\prime}(q, p)=\frac{1}{\eta \mu} H\left(T_{n} q, \mu T_{n}^{-1} p+v\right)
$$

The translation parameter $v=v(H)$ has been included here (optionally) in order to allow elimination of the trivial unstable direction associated with $p$-translations. Instead of working near a RG fixed point, one has to consider a sequence of Hamiltonians like

$$
K_{n}(q, p)=\omega_{n} \cdot p+\frac{m}{2}\left(\Omega_{n} \cdot p\right)^{2}, \quad n=0,1,2 \ldots
$$

with the property that $\mathcal{R}_{n}\left(K_{n}\right)=K_{n+1}$. Define $\widetilde{\mathcal{R}}_{n}=\mathcal{R}_{n-1} \circ \ldots \circ \mathcal{R}_{0}$. One of the goals is to show that if $H_{0}$ is close to $K_{0}$, then $H_{n}-K_{n}$ tends to zero as $n \rightarrow \infty$, where $H_{n}=\widetilde{\mathcal{R}}_{n}\left(H_{0}\right)$. This cannot be expected to work for every irrational rotation number $\alpha_{0}>0$. A technical reason is the following.

Consider e.g. the effect of the step $H \mapsto H\left(T_{n} .,.\right)$ onto a resonant mode whose Fourier index $\nu$ satisfies $\left|\omega_{n} \cdot \nu\right| \leq \sigma_{n}\|\nu\|$. The norm of such a mode changes by a factor (using $\rho=1$ )

$$
f_{n} \approx \exp \left(\left\|T_{n}^{*} \nu\right\|-\|\nu\|\right)
$$

which we would like to be less than one. Now $\left\|T_{n}^{*} \nu\right\| \approx\left\|T_{n}\right\|\left|\omega_{n} \cdot \nu\right|$, and in order to get a negative exponent in (7.6), we need $\sigma_{n}\left\|T_{n}\right\|=\tau_{n}<1$. This yields $\left\|T_{n}^{*} \nu\right\| \lesssim \tau_{n}\|\nu\|$. Given that $\left\|T_{n}^{*} \nu\right\| \geq 1$, we have $\|\nu\| \gtrsim \tau_{n}^{-1}$, and this leads to the bound $f_{n} \lesssim \exp \left(-1 / \tau_{n}\right)$. Next, recall that the elimination procedure $H^{\prime} \mapsto H^{\prime} \circ \mathcal{U}_{H^{\prime}}$ requires that $\mathbb{I}^{-} H^{\prime}$ be bounded in norm by a constant times $\sigma_{n}$. Taking this as the size of the domain for $\mathcal{R}_{n}$, we need

$$
\sigma_{0} f_{0} f_{1} \cdots f_{n-1} \lesssim \sigma_{n}, \quad n=1,2, \ldots
$$


if we wish to have nontrivial Hamiltonians that are infinitely renormalizable. Assume now that we choose $\tau_{n}$ to be a small positive constant, independent of $n$. Then the factors $f_{n}$ are bounded by some fixed positive constant as well. By using that $\sigma_{n} \approx\left\|T_{n}\right\|^{-1} \approx a_{n}^{-1}$, we see that (7.7) can be guaranteed if the sequence $n \mapsto a_{n}$ grows no faster than exponential. (This can be improved by choosing $\tau_{n} \rightarrow 0$.) This is satisfied if $\alpha_{0}$ is Diophantine.

A nonzero vector $\omega \in \mathbb{R}^{d}$ is Diophantine of class $\beta>0$, if there exists a constant $C>0$ such that

$$
|\omega \cdot \nu| \geq C\|\nu\|^{1-d-\beta}, \quad \nu \in \mathbb{Z} \backslash\{0\} .
$$

This set of vectors will be denoted by $\mathrm{DC}(\beta)$. It is well known that $\mathrm{DC}(\beta)$ has full Lebesgue measure in $\mathbb{R}^{d}$; see e.g. [10].

A renormalization group analysis of the type described above was carried out in [81]. It is shown that if $\omega_{0}$ is Diophantine, with $\beta<\sqrt{2}-1$, then $H_{n}-K_{n} \rightarrow 0$ in a space of analytic Hamiltonians, provided that $H_{0}$ is sufficiently close to $K_{0}$. Furthermore, $H_{0}$ has an analytic invariant torus with frequency vector parallel to $\omega_{0}$.

A larger class of irrational numbers is the set of Brjuno numbers. $\alpha_{0}$ is called a Brjuno number if the denominators $q_{n}$ in the rational approximants $p_{n} / q_{n}=\left[a_{0} ; a_{1}, \ldots a_{n}\right]$ of $\alpha_{0}$ satisfy $\sum q_{n}^{-1} \ln \left(q_{n+1}\right)<\infty$. These frequencies were used in an RG analysis of near-constant torus flows [96] and skew flows [97]. For flows on $\mathbb{T}^{2}$ and some other single frequency problems, it is known that the set of Brjuno numbers is exactly the set of frequencies for which one can guarantee linearization.

\subsection{Multidimensional continued fractions}

Here we give a brief description of a multidimensional continued fractions expansion by Khanin, Lopes Dias, and Marklov [70], which is based on the work in [89,73] on geodesic flows on homogeneous spaces.

Consider the one-parameter subgroup of $G=\mathrm{SL}(d, \mathbb{R})$, generated by the matrices

$$
E^{t}=\operatorname{diag}\left(e^{-t}, \ldots, e^{-t}, e^{(d-1) t}\right), \quad t \in \mathbb{R},
$$

Let $F$ be a fundamental domain for the left action of $\Gamma=\operatorname{SL}(d, \mathbb{Z})$ on $G$. Then for every matrix $W_{0} \in G$, and for every time $t \in \mathbb{R}$, there exists a unique matrix $P(t) \in \Gamma$ such that

$$
W(t)=P(t) W_{0} E^{t}
$$

belongs to $F$. A useful invariant under the left action of $\Gamma$ on $G$ is

$$
\delta(W)=\inf _{0 \neq \nu \in \mathbb{Z}^{d}}\left\|\nu^{*} W\right\|
$$

Now consider a special choice of $W_{0}$. Let $\omega=(w, 1)$ be a fixed but arbitrary vector in $\operatorname{DC}(\beta)$, and define $W_{0}$ to be the matrix obtained from the $d \times d$ identity matrix by replacing its last column vector by $\omega$,

$$
W_{0}=\left[\begin{array}{cc}
\mathbf{1} & w \\
0 & 1
\end{array}\right]
$$


In what follows, we assume that the fundamental domain $F$ has been chosen in such a way that it contains $W_{0}$. The Diophantine property of $\omega$ can be used to prove the following bound

$$
\delta\left(W_{0} E^{t}\right) \geq c_{0} e^{-\theta t}, \quad t \geq 0,
$$

where $\theta=\beta /(d+\beta)$. This is one of the main ingredients in the proof of

Theorem 7.1. [70] There exist constants $c_{1}$ and $c_{2}$, depending only on the Diophantine constants $\beta, C$, and on the norm of $\omega$, such that for all $t>0$,

$$
\begin{aligned}
\|W(t)\| & \leq c_{1} \exp \{(d-1) \theta t\} \\
\left\|W(t)^{-1}\right\| & \leq c_{2} \exp \{\theta t\}
\end{aligned}
$$

The information that $W=W(t)$ belongs to $F$ is used as follows. Consider the Iwasawa decomposition of $W=U A K$ of $W$, where $U$ is an upper triangular matrix with diagonal entries $1, A=\operatorname{diag}\left(a_{1}, a_{2}, \ldots, a_{d}\right)$, and $K \in \mathrm{SO}(d)$. It is possible to associate with the fundamental domain $F$ a "Siegel set" $\mathcal{S}$ containing $F$ and being contained in a finite number of $\Gamma$-translates of $F$, which can be characterized by some simple conditions on the matrices $U, A, K$. More specifically, $U$ belongs to a compact fundamental domain for the left action of $\Gamma$ on the group of upper triangular matrices with diagonal entries 1 , and $0<a_{j} \leq 2 \cdot 3^{-1 / 2} a_{j-1}$ for $j>1$.

The crucial step is to prove that $\left|a_{d}\right|$ is bounded from above and below by a constant times $\delta(W)$. Since the norms of $W$ and $W^{-1}$ are of the order of $\left|a_{1}\right|=\left|a_{2} \cdots a_{d}\right|^{-1} \approx$ $\left|a_{d}\right|^{-(d-1)}$ and $\left|a_{d}\right|^{-1}$, respectively, the estimates in Theorem 7.1 now follow from (7.12).

To any "cutting sequence" of times $0=t_{0}<t_{1}<t_{2}<\ldots$, we associate the sequences of matrices $W_{n}=W\left(t_{n}\right)$ and $P_{n}=P\left(t_{n}\right)$. In addition, define $\omega_{0}=\omega$ and

$$
T_{n}=P_{n-1} P_{n}^{-1}, \quad \lambda_{n}=\left\|P_{n} \omega_{0}\right\|, \quad \omega_{n}=\lambda_{n}^{-1} P_{n} \omega_{0},
$$

for $n=1,2, \ldots$.

Let $t_{n}^{\prime}=t_{n}-t_{n-1}$. The following is a straightforward consequence of Theorem 7.1.

Corollary 7.2. [70] There are constants $c_{3}, \ldots, c_{6}>0$, depending only on the Diophantine constants $\beta, C$, and on the norm of $\omega$, such that for all $n>0$,

$$
\begin{aligned}
\left\|P_{n}\right\| & \leq c_{4} \exp \left\{(d \theta+1-\theta) t_{n}\right\}, \\
\left\|P_{n}^{-1}\right\| & \leq c_{3} \exp \left\{(d-1+\theta) t_{n}\right\} \\
\left\|T_{n}\right\| & \leq c_{5} \exp \left\{(d-1)(1-\theta) t_{n}^{\prime}+d \theta t_{n}\right\}, \\
\left\|T_{n}^{-1}\right\| & \leq c_{6} \exp \left\{(1-\theta) t_{n}^{\prime}+d \theta t_{n}\right\} .
\end{aligned}
$$

An important aspect for renormalization is that the matrices $T_{n}$ are hyperbolic whenever $t_{n} \rightarrow \infty$ sufficiently fast, as the following lemma implies.

Lemma 7.3. [70] For all $n>0$, and for all unit vectors $\xi$ that are perpendicular to $\omega_{n-1}$,

$$
\left\|T_{n}^{*} \xi\right\| \leq c_{1} c_{2} \exp \left\{-(1-\theta) t_{n}^{\prime}+d \theta t_{n-1}\right\}
$$


Proof. By definition,

$$
\begin{aligned}
T_{n}^{*} \xi=\left(P_{n}^{-1}\right)^{*} P_{n-1}^{*} \xi & =\left(W_{n}^{-1}\right)^{*} E^{t_{n}} W_{0}^{*} P_{n-1}^{*} \xi \\
& =\left(W_{n}^{-1}\right)^{*} E^{t_{n}^{\prime}}\left[E^{t_{n-1}} W_{0}^{*} P_{n-1}^{*}\right] \xi
\end{aligned}
$$

If $\xi$ is perpendicular to $\omega_{n-1}$ then $P_{n-1}^{*} \xi$ is perpendicular to $\omega_{0}$, and since the last row of $W_{0}^{*}$ is $\omega_{0}$, the last component of $[\ldots] \xi$ is zero. Thus, $[\ldots] \xi$ is an eigenvector of $E^{t_{n}^{\prime}}$ with eigenvalue $e^{-t_{n}^{\prime}}$. Since $[. .]=.W_{n-1}^{*}$, we have

$$
T_{n}^{*} \xi=e^{-t_{n}^{\prime}}\left(W_{n}^{-1}\right)^{*} W_{n-1}^{*} \xi
$$

The estimate (7.16) now follows from Theorem 7.1.

QED

A typical choice for the cutting sequence is $t_{n}=c(1+\alpha)^{n}$, with $c, \alpha>0$ chosen in such a way that the exponential factor in (7.16) is very small; see e.g. Subsection 8.2.

\subsection{Composing different RG transformations}

With each of the vectors $\omega_{n-1}$ and matrices $T_{n}$, we can now associate a RG transformation $\mathcal{R}_{n}$ for vector fields on $\mathbb{T}^{d} \times \mathbb{R}^{\ell}$,

$$
\mathcal{R}_{n}(X)=\eta_{n}^{-1} \mathcal{T}_{n}^{*} \mathcal{U}_{X}^{*} X
$$

Here, $\eta_{n}$ is a time re-normalization factor, that can either be fixed (at the cost of introducing irrelevant expanding directions) or determined by a normalization condition on $\mathcal{R}(X)$. For simplicity, we will choose $\eta_{n}=\lambda_{n-1}^{-1} \lambda_{n}$, independently of the vector field $X$. The trivial RG orbit is given by the constant vector fields $Z_{k}=\left(\omega_{k}, 0\right)$, which satisfy $Z_{n}=\mathcal{R}_{n}\left(Z_{n-1}\right)$ for all $n>0$.

The first task is to show that these RG transformations can be composed properly. To be more precise, denote by $D_{n}$ the domain of $\mathcal{R}_{n}$.

Definition 7.4. Let $B_{0} \subset D_{0}$ be a fixed open neighborhood of $Z_{0}$, and set $\widetilde{\mathcal{R}}_{1}=\mathcal{R}_{1}$. For $n=1,2, \ldots$, define $B_{n}$ to be the set of vector fields $X \in B_{n-1}$ such that $X_{n}=\widetilde{\mathcal{R}}_{n}$ belongs to $D_{n}$, and set $\widetilde{\mathcal{R}}_{n+1}(X)=\mathcal{R}_{n+1}\left(X_{n}\right)$. Then we define the stable manifold for the sequence $\left\{\mathcal{R}_{n}\right\}$ to be the set $\mathcal{W}=\bigcap_{n=0}^{\infty} B_{n}$.

In the next subsection, we will describe the construction of such a stable manifold, in a relatively general setting. The hypotheses can be verified for the action of $\left\{\mathcal{R}_{n}\right\}$ on general vector fields on $\mathbb{T}^{d} \times \mathbb{R}^{\ell}$. We only give a rough description/motivation here and refer to [78] for details. Skew flows will be covered more thoroughly in Section 8.

In order to determine the relative sizes of different quantities, it is convenient to split $\mathcal{R}_{n}$ into a linear part $\mathcal{L}_{n}$ and a nonlinear part $\mathcal{N}_{n}$,

$$
\mathcal{L}_{n} X=\eta_{n}^{-1} \mathcal{T}_{\mu}^{*} \mathbb{I}_{n}^{+} X, \quad \mathcal{N}_{n}(X)=\eta_{n}^{-1} \mathcal{T}_{n}^{*}\left(\mathcal{U}_{X}^{*}-\mathbb{I}_{n}^{+}\right) X
$$


Denote by $\mathbb{E}$ the torus averaging operator. We expect $(\mathbb{I}-\mathbb{E}) X_{n}$ to decrease very rapidly as a function of $n$. As a result, terms involving $\mathcal{N}_{n}$ or $(\mathbb{I}-\mathbb{E}) \mathcal{L}_{n}$ should be extremely small for large $n$. So the hyperbolicity properties of $\widetilde{\mathcal{R}}_{n}$ should be determined essentially by

$$
\mathbb{E} \mathcal{L}_{n} \mathcal{L}_{n-1} \cdots \mathcal{L}_{1}=\lambda_{n}^{-1} \mathcal{T}_{n}^{*} T_{n-1}^{*} \cdots T_{1} \mathbb{E}
$$

Furthermore, we expect $\lambda_{n}^{-1}$ to be a negligible factor in the discussion of these properties.

Recall that $\mathcal{T}_{n}(q, p)=\left(T_{n} q, \mu_{n} S_{n} p\right)$, where $S_{n}$ is either the transposed inverse of $T_{n}$ (if $\ell=d$, and if the class of Hamiltonian vector fields is to be invariant under renormalization) or the identity matrix. Other choices are possible as well, but they seem less natural. For the scaling $\mu_{n}$ there is no natural choice either, unless we wish to impose a nondegeneracy condition on $X_{0}$ and to preserve it under renormalization. In [78] we take $\mu_{n} \rightarrow 0$ at a rate $\left\|T_{n}\right\|^{-\gamma}$, with $\gamma$ sufficiently large (depending on the Diophantine constants for $\omega_{0}$ ). As a result, if $X=\left(X^{\prime}, X^{\prime \prime}\right)$ with $X^{\prime}(q, p)=\mathcal{O}(|p|)$ and $X^{\prime \prime}(q, p)=\mathcal{O}\left(|p|^{2}\right)$, then $X$ is strongly contracted by the operator (7.21). This can be seen from the fact that

$$
\mathcal{T}_{n}^{*} X=\left(T_{n}^{-1} X^{\prime} \circ \mathcal{T}, \mu_{n}^{-1} S_{n}^{-1} X^{\prime \prime} \circ \mathcal{T}\right)
$$

The complementary subspace is spanned by vector fields of the form $X(q, p)=(u, v+M y)$, with $u$ a vector in $\mathbb{R}^{d}, v$ a vector in $\mathbb{R}^{\ell}$, and $M$ a $\ell \times \ell$ matrix. The canonical projection onto this "unstable" subspace will be denoted by $\mathbb{P}$.

Although the nonlinearity $\mathcal{N}_{n}$ contributes very little, it determines the domain of the RG transformation $\mathcal{R}_{n}$. Assume for simplicity that this domain $D_{n}$ is a ball of radius $\rho_{n}$, centered at $Z_{n-1}$. Consider the rescaled RG transformations $R_{n}=\mathcal{V}_{n}^{-1} \circ \mathcal{R}_{n} \circ \mathcal{V}_{n-1}$, where $\mathcal{V}_{k}(X)=Z_{k}+\rho_{k} X$. The following summarizes the expected properties of the RG transformations $\mathcal{R}_{n}$, in a way that relates to the assumptions made in the next subsection, where $\vartheta_{n}$ replaces "very small" and $\varepsilon_{n}$ replaces "tiny".

- $R_{n} \circ \mathbb{P}$ is linear, and its restriction $L_{n}$ to the range of $\mathbb{P}$ has a small inverse.

- $\mathbb{P}_{n} R_{n}-R_{n} \mathbb{P}=\mathbb{P} N_{n}$, and $N_{n}(x)$ is tiny compared to $(\mathbb{I}-\mathbb{E}) x$.

- $(\mathbb{I}-\mathbb{P}) L_{n}=L_{n}(\mathbb{I}-\mathbb{P})$ is very small, so $(\mathbb{I}-\mathbb{P}) R_{n}(x)$ is very small compared to $(\mathbb{I}-\mathbb{P}) x$.

- $\left(\mathbb{I}-\mathbb{E}_{n}\right) L_{n}=L_{n}\left(\mathbb{I}-\mathbb{E}_{n-1}\right)$ is tiny, so $\left(\mathbb{I}-\mathbb{E}_{n}\right) R_{n}(x)$ is tiny compared to $(\mathbb{I}-\mathbb{E}) x$.

\subsection{An invariant manifold theorem}

In this subsection, we describe an invariant manifold theorem for sequences of maps of the type encountered in renormalization. The existence of such a manifold makes the given sequence of maps for many purposes similar to a single dynamical system. In particular, the construction of invariant tori with general Diophantine rotation vectors is essentially the same as the construction described in Section 5 for self-similar rotation vectors; see also the comments at the end of this subsection.

For every integer $n \geq 0$ let $\mathcal{X}_{n}$ be a complex Banach space, and let $\mathbb{E}_{n}, \mathbb{P}_{n}$ be continuous linear projections on $\mathcal{X}_{n}$, satisfying $\mathbb{P}_{n} \mathbb{E}_{n}=\mathbb{E}_{n} \mathbb{P}_{n}=\mathbb{P}_{n}$ and $\left\|\mathbb{E}_{n}\right\|=\left\|\mathbb{I}-\mathbb{E}_{n}\right\|=1$. For each $n>0$, let $R_{n}$ be a bounded analytic map, from an open neighborhood $D_{n-1}$ of the origin in $\mathcal{X}_{n-1}$, to $\mathcal{X}_{n}$, with the following properties: $R_{n} \mathbb{P}_{n-1}$ is linear, and the 
restriction $L_{n}$ of this linear operator to $\mathbb{P}_{n-1} \mathcal{X}_{n-1}$ is invertible. Furthermore, there exist real numbers $\vartheta_{n} \leq \vartheta<1$ and $\varepsilon_{n} \leq \varepsilon=(1-\vartheta) / 4$, such that for all $x \in D_{n-1}$,

$$
\begin{aligned}
\left\|\left(\mathbb{I}-\mathbb{E}_{n}\right) R_{n}(x)\right\| & \leq \varepsilon_{n}\left\|\left(\mathbb{I}-\mathbb{E}_{n-1}\right) x\right\|, \\
\left\|\left(\mathbb{I}-\mathbb{P}_{n}\right) R_{n}(x)\right\| & \leq \vartheta_{n}\left\|\left(\mathbb{I}-\mathbb{P}_{n-1}\right) x\right\|, \\
\left\|\mathbb{P}_{n} R_{n}(x)-L_{n} \mathbb{P}_{n-1} x\right\| & \leq \varepsilon\left\|\left(\mathbb{I}-\mathbb{E}_{n-1}\right) x\right\|, \\
\left\|L_{n}^{-1}\right\| & \leq \vartheta .
\end{aligned}
$$

Consider now the composed maps $\widetilde{R}_{n}=R_{n} \circ R_{n-1} \circ \ldots \circ R_{1}$. The domain of $\widetilde{R}_{1}$ is taken to be $\widetilde{D}_{0}=D_{0}$, and for $n=1,2, \ldots$, the domain $\widetilde{D}_{n}$ of $\widetilde{R}_{n+1}$ is defined inductively as the subset of $\widetilde{D}_{n-1}$ that is mapped into $D_{n}$ by $\widetilde{R}_{n}$.

We will assume that the domain $D_{n-1}$ of $R_{n}$ is given by conditions

$$
\left\|\mathbb{P}_{n-1} x\right\|<1, \quad\left\|\left(\mathbb{I}-\mathbb{P}_{n-1}\right) x\right\|<1, \quad\left\|\left(\mathbb{I}-\mathbb{E}_{n-1}\right) x\right\|<\delta_{n-1},
$$

where $\left\{\delta_{k}\right\}$ is a sequence of positive real numbers, such that $\delta_{k} \geq \varepsilon_{k} \delta_{k-1}$ for all $k>0$.

Theorem 7.5. [78] Let $R_{1}, R_{2}, \ldots$ be a sequence of maps with the properties described above. Then $\mathcal{W}_{0}=\bigcap_{n=0}^{\infty} \widetilde{D}_{n}$ is the graph of an analytic function $W_{0}:\left(\mathbb{I}-\mathbb{P}_{0}\right) D_{0} \rightarrow \mathbb{P}_{0} D_{0}$, satisfying $W_{0}(0)=0$. For every $x \in \mathcal{W}_{0}$,

$$
\begin{aligned}
\left\|\widetilde{R}_{n}(x)\right\| & \leq\left[\vartheta^{(n)}+\varepsilon^{(n)}\right]\left\|\left(\mathbb{I}-\mathbb{P}_{0}\right) x\right\|, \\
\left\|\left(\mathbb{I}-\mathbb{E}_{n}\right) \widetilde{R}_{n}(x)\right\| & \leq \varepsilon^{(n)}\left\|\left(\mathbb{I}-\mathbb{E}_{0}\right) x\right\|,
\end{aligned}
$$

where $\vartheta^{(n)}=\vartheta_{1} \vartheta_{2} \cdots \vartheta_{n}$ and $\varepsilon^{(n)}=\varepsilon_{1} \varepsilon_{2} \cdots \varepsilon_{n}$. Furthermore, if the third condition in (7.23) is strengthened to

$$
\left\|\mathbb{P}_{n} R_{n}(x)-L_{n} \mathbb{P}_{n-1} x\right\| \leq \varphi_{n}\left\|\left(\mathbb{I}-\mathbb{E}_{n-1}\right) x\right\|^{2},
$$

with $\varphi_{n} \delta_{n-1} \leq \varepsilon$, then $D W_{0}(0)=0$, and

$$
\left\|\mathbb{P}_{n} \widetilde{R}_{n}(x)\right\| \leq\left[\vartheta^{(n)}\right]^{2}\left\|\left(\mathbb{I}-\mathbb{P}_{0}\right) x\right\|^{2} .
$$

The proof of this theorem follows the traditional graph transform method: For each $n \geq 0$ we consider a space $\mathcal{F}_{n}$ of analytic families $F: b_{n} \rightarrow \mathcal{X}_{n}$, where $b_{n}$ denotes the open unit ball in $\mathbb{P}_{n} \mathcal{X}_{n}$. Then for $n>0$ and $F \in \mathcal{F}_{n-1}$, define

$$
\Re_{n}(F)=R_{n} \circ F \circ Y_{n, F}^{-1}, \quad Y_{n, F}=\mathbb{P}_{n}\left(R_{n} \circ F\right) .
$$

By construction, $\mathbb{P}_{n} \mathfrak{R}_{n}(F)=I_{n}$, where $I_{n}$ is the inclusion map of $b_{n}$ into $\mathcal{X}_{n}$. Furthermore, $\mathfrak{R}_{n}\left(I_{n-1}\right)=I_{n}$, by the second condition in $(7.23)$.

If $F_{0}$ is any family in the domain of $\mathfrak{R}_{1}$, define $Y_{n}=Y_{n, F_{n-1}}$ and $F_{n}=\mathfrak{R}_{n}\left(F_{n-1}\right)$, for $n=1,2, \ldots$. It can be shown that the orbit $\left\{F_{n}\right\}$ approaches $\left\{I_{n}\right\}$ at a rate $\vartheta^{(n)}$ or 
faster, for any $F_{0}$ in the domain of $\mathfrak{R}_{1}$. The re-parametrization maps $Y_{n}$ approach $L_{n}$ at the same rate, which allows us to define

$$
z_{n}=z_{n}\left(F_{0}\right)=\lim _{m \rightarrow \infty}\left(Y_{n+1}^{-1} \circ Y_{n+2}^{-1} \circ \ldots \circ Y_{m}^{-1}\right)(0)
$$

Notice that $R_{n}\left(F_{n-1}\left(z_{n-1}\right)\right)=F_{n}\left(z_{n}\right)$ for all $n>0$, and in particular, $F_{0}\left(z_{0}\right)$ is infinitely renormalizable. The function $W_{0}$ described in Theorem 7.5 is now defined by setting $W_{0}(x)=z_{0}(s \mapsto x+s)$ for every $x \in\left(\mathbb{I}-\mathbb{P}_{0}\right) D_{0}$.

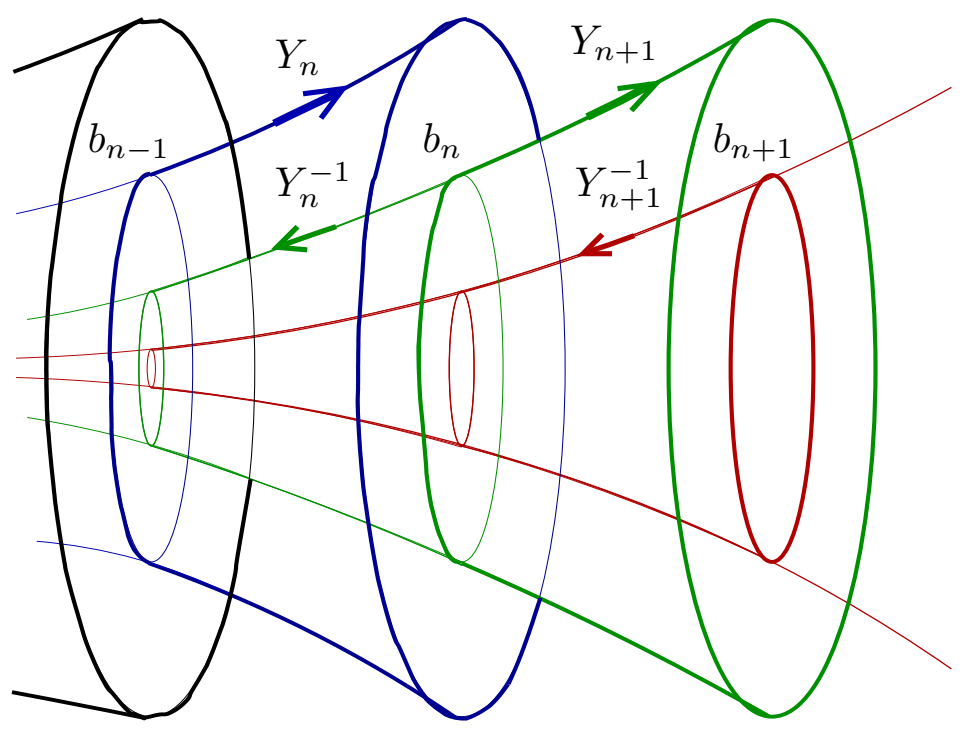

Fig. 11. The reparametrization maps $Y_{n}$

More details, and an application of this theorem to the construction of invariant $d$-tori for analytic flows on $\mathbb{T}^{d} \times \mathbb{R}^{\ell}$ can be found in [78]. Following the construction described in Subsection 5.2, every vector field $X$ on the local "stable" manifold $\mathcal{W}$ at $Z_{0}$ can be shown to have a analytic invariant torus with frequency vector $\omega_{0}$ (assumed to be Diophantine). This translates immediately into a result for $m$-parameter families, where $m$ is the codimension of $\mathcal{W}$. If the vector fields are restricted to an invariant subclass, such as the ones described in subsection 2.4, then the number of required parameter reduces to the number of unstable directions in this subclass. Notice that a trivial reduction by 1 can always be achieved via a time scaling $X \mapsto \tau X$. A further reduction is possible (via translations or scaling in $\mathbb{R}^{\ell}$ ) if the analysis is restricted to vector fields satisfying a nondegeneracy assumption. In the Hamiltonian case, the number of necessary parameters can be reduced to zero in this way, as was explained in Subsection 5.1. 


\section{Reduction of skew flows}

Here we apply the framework developed in Section 7 to a concrete case: the reducibility problem for near-linear skew flows. In addition, we will describe a way of dealing with vector fields near $(\omega, A)$, with $A$ constant but not necessarily equal to zero. The analysis of the case $\operatorname{SL}(2, \mathbb{R})$ also involves a reduction of the number of unstable directions that is not of the type mentioned above. The work described in this section is from reference [79].

\subsection{A general result}

The first result describes a general class of vector fields $X=(\omega, f$.$) that are reducible to$ the trivial vector field $(\omega, 0)$. See Subsections 2.5 and 3.1 for a definition of reducibility, and for notation.

In what follows, the constants $\beta$ and $C$ in the Diophantine condition (7.8) are considered fixed. Define

$$
\gamma_{0}(\beta)=(d+\beta)[1+2 \beta+2 \sqrt{\beta[1+\beta-1 /(d+\beta)]}]-1 .
$$

Theorem 8.1. [79] Given $\gamma \geq \gamma_{2}>\gamma_{0}(\beta)$, there exists an open neighborhood $B$ of the origin in $\mathcal{F}_{\gamma}$, and for each Diophantine unit vector $\omega$ satisfying (7.8) a manifold $\mathcal{W}$ in $B$, such that the following holds. $\mathcal{W}$ is the graph of an analytic map $W:(\mathbb{I}-\mathbb{E}) B \rightarrow \mathbb{E} B$, which vanishes together with its derivative at the origin, and which takes values in $\mathcal{A}_{\gamma}$ when restricted to $\mathcal{A}_{\gamma}$. Every function $f$ on $\mathcal{W}$ is reducible to zero. The corresponding change of coordinates $V$ belongs to $\mathcal{F}_{\varepsilon}$ and depends analytically on $f$, where $\varepsilon=\gamma-\gamma_{2}$. If in addition, $f \in \mathcal{A}_{\gamma}$, then $V$ belongs to $\mathcal{G}_{\varepsilon}$, and if $f$ is the restriction to $\mathbb{T}^{d}$ of an analytic function, then so is $V$.

Here, a function $\psi$ defined on $\mathcal{W}$ is said to be analytic if $\psi \circ W$ is analytic on the domain of $W$.

This theorem can also be applied to vector fields $Y=(w, g$.$) , with g$ is close to a constant matrix $A$, but not necessarily small. Assume that $A \in \mathfrak{A}$ admits a spectral decomposition $A=\kappa \cdot J=\kappa_{1} J_{1}+\ldots+\kappa_{\ell} J_{\ell}$, where the $J_{j}$ are linearly independent mutually commuting matrices in $\mathfrak{A}$, with the property that $t \mapsto \exp \left(t J_{j}\right)$ has fundamental period $2 \pi$. The vector $\kappa \in \mathbb{R}^{\ell}$ will be referred to as the frequency vector of $A$.

In order to see how Theorem 8.1 can be applied to $g \approx A$, we start with a skew system $Y=(w, g$.$) on \mathbb{T}^{m} \times \mathfrak{G}$, and then take $d=m+\ell$. Consider the associated function

$$
f(q)=e^{-r \cdot J} g(x) e^{r \cdot J}-\kappa \cdot J, \quad q=(x, r) \in \mathbb{T}^{m} \times \mathbb{T}^{\ell} .
$$

Notice that $f \approx 0$. If $Y$ is regarded as a vector field on $\mathcal{M}$ by identifying $w$ and $x$ with $(w, 0)$ and $(x, 0)$, respectively, then the above relation between $g$ and $f$ can be written as

$$
f=\Theta^{\star} g, \quad \Theta(q, y)=\left(q, e^{r \cdot J} y\right) .
$$

If $\omega=(w, \kappa)$ is Diophantine of type (7.8) and $g$ belongs to the manifold $\Theta_{\star} \mathcal{W}$, then the flow for $X=(\omega, f$.$) can be trivialized with a change of coordinates V$, as described in 
Theorem 8.1. The same now holds for $g$. However, the corresponding change of variables $V_{g}(q)=V(q) e^{-r \cdot J}$ is not of the desired form, since it still depends on the coordinates $r_{j}$. But as we will show later,

$$
\Psi_{Y}^{t}(x)=V_{g}(x+t \omega)^{-1} V_{g}(x)=V(x+t w)^{-1} e^{t C} V(x),
$$

for some matrix $C \in \mathfrak{A}$ with frequency vector $\kappa$, provided that $V$ is differentiable. What remains to be shown, in specific cases, is that the space of functions of the type (8.2) has a reasonable intersection with the manifold $\mathcal{W}$.

This procedure can be characterized as transforming some (quasi)periodic motion on $\mathfrak{G}$ into motion on an extended torus. This makes it possible to treat all frequencies of the system in a unified way. In the case $\mathfrak{G}=\mathrm{SL}(2, \mathbb{R})$, which will be discussed in Subsection 8.4 , it also has the advantage that the analysis of near-constant skew flows $Y=(\omega, g$. can be reduced to a purely local analysis near $f \equiv 0$.

\subsection{The stable manifold}

With each of the vectors $\omega_{n-1}$ and matrices $T_{n}$ obtained in Subsection 7.2, we can associate a RG transformation for skew flows: $\mathcal{N}_{n}(f)=\eta_{n}^{-1} \mathcal{T}_{n}^{\star} \mathcal{U}_{f}^{\star} f$, where $\eta_{n}=\lambda_{n-1}^{-1} \lambda_{n}$. Let us reformulate Theorem 3.3 for each of these RG transformations. The condition (3.4) reads

$$
2 \sigma_{n}\left\|T_{n}\right\|<\tau_{n}, \quad\left\|T_{n}^{*} \xi\right\| \leq \frac{\tau_{n}}{2}\|\xi\|, \quad \xi \in \omega_{n-1}^{\perp}
$$

and Theorem 3.3 becomes

Theorem 8.2. Assume that $\sigma_{n}$ and $\tau_{n}$ satisfy (8.5). Let $f=C+h$, with $C$ constant and $\mathbb{E} h=0$. If $\|C\|<\sigma_{n} / 6$ and $\|h\|<2^{-9} \sigma_{n}$, then

$$
\mathcal{N}_{n}(f)=\eta_{n}^{-1}[C+\tilde{h}], \quad\|\tilde{h}\| \leq \frac{3}{2} \tau_{n}^{\gamma}\|h\|, \quad|\mathbb{E} \tilde{h}| \leq 2^{4} \sigma_{n}^{-1} \tau_{n}^{\gamma}\|h\|^{2}
$$

$\mathcal{N}_{n}$ is analytic on the region determined by the given bounds on $C$ and $h$.

The goal now is to compose these RG transformations. The domain $B_{n}$ of $\mathcal{N}_{n}$ is defined to be the ball of radius $2^{-9} \sigma_{n}$ in $\mathcal{A}$, centered at zero. Ignoring the constant component $C$ of $f$, the range of $\mathcal{N}_{n}$ is by a factor $<2 \eta_{n}^{-1} \tau_{n}^{\gamma}$ smaller than its domain. By multiplying up these factors, we get the following condition for the composability of the transformations $\mathcal{N}_{n}$.

$$
\lambda_{n}^{-1} \prod_{j=1}^{n}\left(4 \tau_{j}^{\gamma_{1}}\right) \cdot \sigma_{1} \leq \sigma_{n+1}
$$

Lemma 8.3. Given $\gamma_{1}>\gamma_{0}(\beta)$, there exist two sequences $n \mapsto \sigma_{n}$ and $n \mapsto \tau_{n}$ of positive real numbers less than one, both converging to zero, such that the following holds. If $\omega_{0}$ is a unit vector in $\mathbb{R}^{n}$ satisfying the Diophantine condition (7.8), then there exists a sequence 
$n \mapsto P_{n}$ of unimodular integer matrices, such that with $T_{n}$ and $\lambda_{n}$ as defined in (7.14), the conditions (8.5) and (8.7) hold for $n=1,2, \ldots$

It is straightforward to verify these estimates by using the results of [70] described in Subsection 7.2, with a cutting sequence of the form $t_{n}=c(1+\alpha)^{n}$, and with the choice

$$
\sigma_{n}=\sigma_{0} e^{-d t_{n}^{\prime}}, \quad \tau_{n}=\tau_{0} \exp \left\{-[(d-1) \theta+1] t_{n}^{\prime}+d \theta t_{n}\right\},
$$

For the factor $\lambda_{n}^{-1}$ in (8.7), it suffices to use the trivial bound $\lambda_{n}^{-1} \leq\left\|P_{n}^{-1}\right\|$.

Consider now the composed RG transformations $\widetilde{\mathcal{N}}_{n}=\mathcal{N}_{n} \circ \ldots \circ \mathcal{N}_{1}$, with domains defined as usual. Let $r_{0}=2^{-11} \sigma_{1}$. By Theorem 8.2 and Lemma 8.3, the question whether the function $f_{n-1}=\widetilde{\mathcal{N}}_{n-1}\left(f_{0}\right)$ belongs to the domain of $\mathcal{N}_{n}$ only depends on its average $\mathbb{E} f_{n-1}$. To be more precise, given an open set $B \subset B_{1}$ containing zero, define $\widetilde{B}_{0}=B$ and

$$
\widetilde{B}_{n}=\left\{f \in \widetilde{B}_{n-1}:\left\|\mathbb{E} f_{n-1}\right\|<r_{n-1}\right\}, \quad r_{n}=\lambda_{n}^{-1} 4^{-n} \pi_{n}^{\gamma_{1}} r_{0},
$$

for $n=1,2, \ldots$. Then $\widetilde{B}_{n}$ is contained in the domain of $\widetilde{\mathcal{N}}_{n}$.

Theorem 8.4. [79] If $\gamma>\gamma_{1}$ then there exists a non-empty open neighborhood $B$ of the origin in $\mathcal{F}$, such that $\mathcal{W}=\bigcap_{n=0}^{\infty} \widetilde{B}_{n}$ is the graph of an analytic function $W:(\mathbb{I}-\mathbb{E}) B \rightarrow$ $\mathbb{E} B$. Both $W$ and its derivative vanish at the origin.

This is now essentially a corollary of Theorem 7.5, if we set $R_{n}(f)=r_{n}^{-1} \mathcal{N}_{n}\left(r_{n-1} f\right)$. The projections used are are simply $\mathbb{P}_{n}=\mathbb{E}_{n}=\mathbb{E}$, and the parameters are $\varepsilon_{n}=\vartheta_{n}=\tau_{n}^{\gamma-\gamma_{1}}$ and $\delta_{n}=2^{-n}$.

\subsection{Conjugacy to a linear flow}

In this subsection we outline a proof for the remaining parts of Theorem 8.1 concerning the reducibility of vector fields on the manifold $\mathcal{W}$.

Consider $f_{0} \in \mathcal{W}$, and let $f_{n}=\widetilde{\mathcal{N}}_{n}\left(f_{0}\right)$. In order to simplify notation, the transformation $\mathcal{U}_{f_{n}}$ (defined in Subsection 3.2) and the flow $\Psi_{\left(\omega_{n}, f_{n}\right)}$ will be denoted by $U_{n}$ and $\Psi_{n}$, respectively. For $m>n \geq 0$, define

$$
V_{m, n}(q)=U_{m-1}\left(T_{m-1}^{-1} \cdots T_{n+1}^{-1} q\right) \cdots U_{n+1}\left(T_{n+1}^{-1} q\right) U_{n}(q) .
$$

Then the flows for $f_{m}$ and $f_{n}$ are related by the equation

$$
\Psi_{n}^{t}(q)=V_{m, n}\left(q+t \omega_{n}\right)^{-1} \Psi_{m}^{\eta_{m} \ldots \eta_{n+1} t}\left(T_{m}^{-1} \ldots T_{n+1}^{-1} q\right) V_{m, n}(q) .
$$

We would like to show that the three terms on the right hand side of this equation each have limits as $m \rightarrow \infty$, with the $\Psi$ term in the middle converging to the identity. This would yield the desired conjugacy. Due to the matrices $T_{n}^{-1}$ involved, these limits will have to be considered in $\mathcal{G}_{0}$, using that $\left\|f \circ T_{n}^{-1}\right\|_{0}=\|f\|_{0} \leq\|f\|_{\gamma}$.

The time $t$ map $\Psi_{X}^{t}$ associated with a vector field $X=(\omega, f)$ can be estimated as usual by applying the contraction mapping principle to the corresponding integral equation, and 
then using the group property. The resulting bound is $\left\|\Psi_{X}^{t}-\mathrm{I}\right\|_{\gamma} \leq e^{\|t f\|_{\gamma}}-1$. And for changes of variables, we can use the bound

$$
\left\|\mathcal{U}_{f}-\mathrm{I}\right\|_{\gamma} \leq \exp \left(3 \sigma^{-1}\|(\mathbb{I}-\mathbb{E}) f\|_{\gamma}\right)-1
$$

which is obtained from the results in Subsection 3.2 and holds for $f$ in the domain of $\mathcal{N}$.

These estimates can now be combined with the bound

$$
\sigma_{n+1}^{-1}\left\|f_{n}\right\|_{\gamma} \leq 2^{-n} \pi_{n}^{\gamma-\gamma_{1}} \sigma_{1}^{-1}\left\|f_{0}\right\|_{\gamma}
$$

obtained from Theorem 8.2 and Lemma 8.3, to prove the following

Lemma 8.5. If $f_{0} \in \mathcal{W}$, then the limit $V_{n}=\lim _{m \rightarrow \infty} V_{m, n}$ exist in $\mathcal{G}_{0}$ and satisfies

$$
\left\|V_{n}-\mathrm{I}\right\|_{0} \leq 2^{4-n} \pi_{n}^{\gamma-\gamma_{1}} \sigma_{1}^{-1}\left\|f_{0}\right\|_{\gamma}
$$

Furthermore, the maps $f_{0} \mapsto V_{n}$ are analytic, and

$$
\Psi_{n}^{t}(q)=V_{n}\left(q+t \omega_{n}\right)^{-1} V_{n}(q), \quad t \in \mathbb{R}
$$

This lemma will now be applied for $\gamma=\gamma_{2}$, with $\gamma_{2}>\gamma_{1}$ fixed. If $f$ has some excess regularity, that is, if $f$ belongs to $\mathcal{A}_{\gamma}$ with $\gamma>\gamma_{2}$, then this excess regularity is inherited by $V_{0}$. The mechanism is analogous to the one described in Subsection 5.1. To be more specific, consider the torus translations $\left(\mathcal{J}_{p} f\right)(q)=f(q+p)$. It is straightforward to check that the manifold $\mathcal{W}$ is invariant under $\mathcal{J}_{p}$, and that the map $f_{0} \mapsto V_{0}$ commutes with $\mathcal{J}_{p}$. Denote by $\mathcal{H}$ the map that associates to each $f$ in the domain of $W$ via $f_{0}=f+W(f)$ the value $V_{0}(0)$. Then

$$
V_{0}(p)=\mathcal{H}\left(\mathcal{J}_{p} f\right), \quad p \in \mathbb{T}^{d}
$$

Given that $\mathcal{H}$ is analytic, this identity links the regularity of $V_{0}$ to the regularity of $p \mapsto \mathcal{J}_{p} f$ as a family in $\mathcal{F}_{\gamma_{2}}$. More precisely, we have

Lemma 8.6. Let $\gamma \geq \gamma_{2}>\gamma_{1}$ and $\varepsilon=\gamma-\gamma_{2}$. If $f_{0} \in \mathcal{W}$ then the function $V_{0}$ described in Lemma 8.5 belongs to $\mathcal{G}_{\varepsilon}$ and has a directional derivative $D_{\omega_{0}} V_{0}$ in $\mathcal{F}_{\varepsilon}$. As elements of $\mathcal{F}_{\varepsilon}$, both $V_{0}$ and $D_{\omega_{0}} V_{0}$ depend analytically on $f_{0}$. Furthermore, if $f_{0}$ is the restriction to $\mathbb{T}^{d}$ of an analytic function, then so is $V_{0}$.

Lemma 8.5 and Lemma 8.6 imply the statements in Theorem 8.1 concerning the reducibility of functions $f \in \mathcal{W}$ and the regularity of the corresponding change of coordinates. The fact that $V$ takes values in $\mathfrak{G}$ (if $\mathfrak{G}$ is a proper Lie subgroup of $\mathrm{GL}(n, \mathbb{C}$ ), or a Lie subgroup of $\operatorname{GL}(n, \mathbb{R})$ ) whenever $f$ takes values in the corresponding algebra $\mathfrak{A}$, follows by construction; see also the remark at the end of Subsection 3.2.

As was described in Subsection 8.1, Theorem 8.1 can also be applied to vector fields $Y=(w, g$.$) , with g$ close to a nonzero constant matrix $A=\kappa \cdot J$. However, a direct application reduces $Y$ as a skew system over $\mathbb{T}^{d}$ instead of $\mathbb{T}^{m}$, which involves extra frequencies. The following result shows that these frequencies can be factored out. 
Lemma 8.7. Let $\gamma \geq \gamma_{2}+1$ with $\gamma_{2}>\gamma_{1}$. Assume that $f \in \mathcal{A}_{\gamma}$ is of the form (8.2) and belongs to $\mathcal{W}$. Let $V=V_{0}$, corresponding to $f_{0}=f$. Then the flow for $Y=(w, g$.) is given by equation (8.4), for some $C \in \mathfrak{A}$. The corresponding map $f \mapsto C$ is analytic.

Proof. The first equality in (8.4) follows from Lemma 8.5 and the definition (8.3). Let

$$
\phi^{t}(x)=V(x+t w) \Psi_{Y}^{t}(x) V(x)^{-1}=V(x+t w) e^{t A} V(x+t \omega)^{-1},
$$

for $t \in \mathbb{R}$ and $x \in \mathbb{T}^{m}$. Notice that $\phi$ is the flow for a skew system $Z=\left(w, h\right.$.) on $\mathbb{T}^{m} \times \mathfrak{G}$, and since $V \in \mathcal{G}_{1}$ by Lemma 8.6, the function $h$ belongs to $\mathcal{A}_{0}$.

Consider now an arbitrary sequence $\left\{t_{j}\right\}$ such that $t_{j} \kappa \rightarrow 0$ on the torus $\mathbb{T}^{\ell}$, as $j \rightarrow \infty$. Then $\exp \left(t_{j} \kappa \cdot J\right) \rightarrow \mathrm{I}$. Furthermore, $\operatorname{dist}\left(t_{j} \omega, t_{j} w\right) \rightarrow 0$ on the torus $\mathbb{T}^{d}$, and since $V$ is of class $\mathrm{C}^{1}$, we have $\phi^{t+t_{j}}(x) \rightarrow \phi^{t}(x)$ uniformly in $x$, if $t=0$. By the cocycle identity for the flow $\phi$, the same holds for any $t \in \mathbb{R}$, and the convergence is uniform in $t$. This implies (see e.g. [53]) that the function $t \mapsto \phi^{t}(x)$ is periodic or quasiperiodic, with frequencies in $F(\kappa)=\left\{\kappa_{1}, \ldots, \kappa_{\ell}\right\}$. As a result,

$$
h(x+t w)=\dot{\phi}^{t}(x) \phi^{t}(x)^{-1}
$$

is also periodic or quasiperiodic in $t$, with frequencies in $F(\kappa)$. But the frequency module of $t \mapsto h(x+t w)$ is clearly a subset of $F(w)=\left\{w_{1}, \ldots, w_{m}\right\}$, and since $F(w) \cap F(\kappa)$ is empty, $h$ has to be constant. Setting $C=h$, we obtain $\phi^{t}(x)=e^{t C}$, and the identity (8.4) now follows from (8.16). A computation of $h(x)$ from the equations (8.17) and (8.16) yields $C=V A V^{-1}-\left(D_{\kappa} V\right) V^{-1}$, evaluated at $x$. This identity (between matrices, if $x$ is fixed), together with Lemma 8.6, shows that $C$ depends analytically on $f$.

QED

\subsection{The special case $\mathfrak{G}=\mathrm{SL}(2, \mathbb{R})$}

Consider now the group $\mathfrak{G}=\operatorname{SL}(2, \mathbb{R})$ and the corresponding Lie algebra $\mathfrak{A}$ of traceless $2 \times 2$ matrices. In this case, Theorem 8.1 yields a stable manifold of codimension 3 . On the other hand, it is known (at least in the analytic setting) that reducibility is a codimension 1 phenomenon, governed by the so-called fibered rotation number. The goal here is to describe how this fits in the framework of renormalization.

Consider first the flow for $Y=(w, g$.$) on the product of \mathbb{T}^{d-1}$ with $\mathbb{R}^{2} \backslash\{0\}$,

$$
\dot{v}(t)=g\left(x_{0}+t w\right) v(t), \quad v(0)=v_{0} .
$$

Denote by $\alpha(t)$ the angle between $v(t)$ and some fixed unit vector $u_{0}$, and let $\alpha_{0}=\alpha(0)$. Then the lift of this angle to $\mathbb{R}$ evolves according to the equation

$$
\dot{\alpha}(t)=-\left\langle e^{-\alpha(t) J} J g\left(x_{0}+t w\right) e^{\alpha(t) J} u_{0}, u_{0}\right\rangle, \quad \alpha(0)=\alpha_{0},
$$

where $\langle.,$.$\rangle denotes the standard inner product on \mathbb{R}^{2}$. Here, and in the remaining part of this section, $J=\left[\begin{array}{rr}0 & -1 \\ 1 & 0\end{array}\right]$. Assuming that the components of $w$ are rationally independent, we can define the fibered rotation number of $Y$,

$$
\varrho(Y)=\lim _{t \rightarrow \infty} \frac{\alpha(t)}{t} .
$$


This limit exists and is independent of the initial conditions $x_{0}$ and $\alpha_{0}$ [66]. If $w$ is fixed, we will also write $\varrho(g)$ in place of $\varrho(Y)$.

From the definition of $\Theta$, we see that $\varrho(Y)=\kappa$ if and only if $\varrho(X)=0$. Thus, we may restrict our analysis to skew flows with fibered rotation number zero. Theorem 8.1 deals with precisely such flows. However, the functions (8.2) are of a particular type, and

more can be said in this case. Denote by $\mathcal{A}_{\gamma}^{0}$ the subspace of functions $g \in \mathcal{A}_{\gamma}$ with the property that $g(q)=g(x)$ for all $q=(x, r)$ in $\mathbb{T}^{d-1} \times \mathbb{T}^{1}$.

Theorem 8.8. [79] Given $\gamma \geq \gamma_{2}>\gamma_{0}(\beta)$ and $a>0$, the following holds for some $R>0$. Consider a constant skew system $(w, A)$ on $\mathbb{T}^{d-1} \times \mathfrak{G}$, for a matrix $A \in \mathfrak{A}$ that has purely imaginary eigenvalues, say $\pm \kappa i$. Assume that $\omega=(w, \kappa)$ satisfies the Diophantine condition (7.8), and that $\|A\| \leq a|\kappa|\|\omega\|$. Then there exists an open neighborhood $B_{0}$ of the constant function $x \mapsto A$ in $\mathcal{A}_{\gamma}^{0}$, containing a ball of radius $R$ centered at this function, such that for any $g \in B_{0}$, the one-parameter family $\lambda \mapsto g+\lambda A$ contains a unique member in $B_{0}$, say $g^{\prime}$, whose associated skew flow has a fibered rotation number $\kappa$. If $\gamma-\gamma_{2}=\varepsilon \geq 1$, then $g^{\prime}$ is reducible to a constant $C \in \mathfrak{A}$, as described by equation (8.4), via a change of coordinates $V \in \mathcal{G}_{\varepsilon}$. Furthermore, the function $g^{\prime}$, and (if $\varepsilon \geq 1$ ) the quantities $C$ and $V$, depend real analytically on $g$.

This theorem is proved by first performing a change of coordinates $g \mapsto L^{-1} g L$ with $L \in \mathfrak{G}$, such that $L^{-1} A L=\kappa J$, followed by a constant scaling $Y \mapsto c Y$ of the resulting skew system, which converts $(w, \kappa)$ to a unit vector. After that, the task is reduced via the map $\Theta$ to the study of vector fields $X=(\omega, f$.$) with f$ of the type (8.2). Thus, in view of Theorem 8.1 and Lemma 8.7 , it suffices to prove (besides real analyticity) that the family $\lambda \mapsto f+\lambda J$ intersects the manifold $\mathcal{M}$ in exactly one point, characterized by $\varrho(f+\lambda J)=0$. A sketch of the proof will be given at the end of the next subsection.

The main difficulty with this approach is that the subspace $\mathcal{A}^{1}$ of functions $f \in \mathcal{A}$ of the form (8.2) is not invariant under renormalization. Notice that this subspace can also be characterized by the identity $f(q+(0, r))=e^{-r J} f(q) e^{r J}$. Thus, that the torusaverage of $f \in \mathcal{A}^{1}$ is necessarily a constant multiple of $J$. This may seem to explain the statement about one-parameter families in Theorem 8.8. However, this property is neither invariant under renormalization, nor does is guarantee that the flow for $X=(\omega, f$.) remains bounded.

\subsection{Excluding hyperbolicity}

The three unstable directions under renormalization correspond to elliptic, parabolic, and hyperbolic behavior of the flow. The goal is to show that a vector field $X=(\omega, f$.$) , with$ $f \in \mathcal{A}^{1}$ close to zero, can only generate an elliptic flow, by excluding e.g. the possibility that the renormalized functions $f_{n}=\widetilde{\mathcal{N}}_{n}\left(f_{0}\right)$ have the following property.

Definition 8.9. Let $S^{1}$ be the set of unit vectors in $\mathbb{R}^{2}$. We say that a vector field $X=(\omega, f$.$) has the expanding cone property if for every q \in \mathbb{T}^{d}$, there exists an open cone $\mathcal{C}(q)$ in $\mathbb{R}^{2}$ not intersecting its negative, with vertex at zero, and a unit vector $u(q)$ in this cone, such that the following holds. The map $q \mapsto S^{1} \cap \mathcal{C}(q)$ defines two continuous functions from $\mathbb{T}^{d}$ to $S^{1}$. The function $q \mapsto u(q)$ is continuous as well, and homotopic to a 
constant. Furthermore, for every $q \in \mathbb{T}^{d}$, the cone $\Psi_{X}^{t}(q) \mathcal{C}(q)$ is contained in $\mathcal{C}(q+t \omega)$ for all $t>0$, and the length of $\Psi_{X}^{t}(q) u(q)$ tends to infinity as $t \rightarrow \infty$.

The usefulness of the expanding cone property stems from the fact that it is invariant under coordinate changes of the form (3.2) or (2.26), with $V$ continuous and homotopic to the identity. A simple condition that implies this property is the following.

Proposition 8.10. Assume that $f: \mathbb{T}^{d} \rightarrow \mathfrak{A}$ is continuous and of the form $f=C+h$, with $C \in \mathfrak{A}$ symmetric and $\|h(q)\|<\|C\| / 4$ for all $q \in \mathbb{T}^{d}$. Then $X=(\omega, f$.) has the expanding cone property.

This proposition is proved first for $h=0$, where it is trivial, and then a perturbative argument is used for $\|h(q)\|<\|C\| / 4$.

Lemma 8.11. If $f$ belongs to $\mathcal{A}^{1}$ then $X=(\omega, f$.) cannot have the expanding cone property.

Proof. Consider first an arbitrary $f \in \mathcal{A}$ such that $X=(\omega, f$.) has the expanding cone property. Let $q \in \mathbb{T}^{d}$ be fixed. Using the notation of Definition 8.9, denote by $A(q)$ the set of all nonzero $v_{0} \in \mathbb{R}^{2}$ such that $v(t)=\Psi_{X}^{t}(q) v_{0}$ belongs to $\mathcal{C}(q+t w)$ for some (and thus each sufficiently large) positive $t$. This set is clearly open. Notice that if $v_{0}$ is any nonzero vector in $\mathbb{R}^{2}$, with the property that $v(t)=\Psi_{X}^{t}(q) v_{0}$ tends to infinity as $t \rightarrow \infty$, then $v_{0}$ belongs to either $A(q)$ or $-A(q)$. This follows from the fact that $\Psi_{X}^{t}(q)$ is area-preserving (so the angle between $v(t)$ and $\Psi_{X}^{t}(q) u(q)$ has to approach zero), and that the opening angles of our cones are bounded away from zero. Thus, given that the two disjoint open sets $\pm A(q)$ cannot cover all of $\mathbb{R}^{2} \backslash\{0\}$, it is not possible that $|v(t)| \rightarrow \infty$ as $t \rightarrow \infty$, for every nonzero $v_{0} \in \mathbb{R}^{2}$.

Assume now for contradiction that $f$ belongs to $\mathcal{A}^{1}$. Define $z_{r}(x)=e^{r J} u(q)$, with $u$ as described in Definition 8.9. Then $\Psi_{Y}^{t}(x) z_{r}(x)=e^{(r+t \kappa) J} \Psi_{X}^{t}(q) u(q)$ tends to infinity as $t \rightarrow \infty$. But as $r$ increases from 0 to $2 \pi$, the vectors $z_{r}(x)$ cover all of $S^{1}$, since $u$ is homotopic to a constant function. This implies that $\Psi_{Y}^{t}(x) v_{0}$ tends to infinity (in length) for each nonzero $v_{0} \in \mathbb{R}^{2}$, which was shown above to be impossible.

QED

Now we are ready to renormalize and to continue our sketch of the proof of Theorem 8.8. Denote by $\mathfrak{J}$ the one-dimensional subspace of $\mathfrak{A}$, consisting of real multiples of the matrix $J$.

The idea is to consider the family $F(s)=s+(\mathbb{I}-\mathbb{E}) f$ associated with a function $f \in \mathcal{A}^{1}$ close to zero, where $s \in \mathcal{A}$, and to show that the parameter value $s=z_{0}$, where this family intersects the stable manifold $\mathcal{W}$, belongs to $\mathfrak{J}$. This is proved by contradiction: If the intersection takes place at a point $s$ outside $\mathfrak{J}$, then by renormalizing the family $F$ as in the proof of the stable manifold theorem (see subsection 7.4), we can find $n>0$ and $s_{0} \in \mathfrak{J}$, such that $F_{n}\left(s_{n}\right)=\mathfrak{N}_{n}\left(F\left(s_{0}\right)\right)$ is much closer to its average value $s_{n}$ than this value is to $\mathfrak{J}$. By using that the re-parametrization maps $Y_{n}$ for skew flows are very close to multiples of the identity, we can in fact choose $s_{0}$ in such a way that $s_{n}$ is symmetric. Then $F_{n}\left(s_{n}\right)$ has the expanding cone property. The same is true for $F\left(s_{0}\right)$, since this property is preserved under coordinate changes of the form (3.2) or (2.26). But $F\left(s_{0}\right)$ belongs to $\mathcal{A}^{1}$, 
and we get a contradiction with Lemma 8.11. This shows that $z_{0} \in \mathfrak{J}$. And Lemma 8.5 implies that $\varrho\left(F\left(z_{0}\right)\right)=0$.

Finally, assume for contradiction that $\varrho\left(F\left(s_{0}\right)\right)=0$ for some $s_{0} \in \mathfrak{J}$ different from $z_{0}$, By renormalizing $F$ we can achieve $\left\|s_{n}-z_{n}\right\|>2\left\|z_{n}\right\|$, which implies (using again that the maps $Y_{n}$ are close to multiples of the identity) that $F_{n}\left(s_{n}\right)$ is close enough to an antisymmetric matrix to have a strictly positive determinant. But then $F_{n}\left(s_{n}\right)$ cannot have a vanishing fibered rotation number, and the same is true for $F\left(s_{0}\right)$. This shows that the family $f \mapsto f+\lambda J$ intersects $\mathcal{W}$ at a unique point, characterized by $\varrho(f+\lambda J)=0$.

For details we refer to the proof of Lemma 7.5 in [79].

\section{Acknowledgments}

This review grew out of lecture notes for a mini-course given at the Fields Institute in Toronto during the Fall 2006 thematic program on Renormalization and Universality in Mathematics and Mathematical Physics. The author would like to thank the organizers of this program, especially Mikhail Lyubich and Michael Yampolsky, as well as the Fields Institute and its staff, for their generous support and hospitality.

\section{References}

[1] J.J. Abad, H. Koch, Renormalization and periodic orbits for Hamiltonian flows. Commun. Math. Phys. 212, 371-394 (2000).

[2] J.J. Abad, H. Koch, and P. Wittwer, A Renormalization Group for Hamiltonians: Numerical Results. Nonlinearity 11, 1185-1194 (1998).

[3] A. Apte, A. Wurm and P.J. Morrison, Renormalization and destruction of $1 / \gamma^{2}$ tori in the standard nontwist map. Chaos 13, 421-433 (2003).

[4] A. Apte, A. Wurm and P.J. Morrison, Renormalization for breakup of invariant tori. Physica D 200, 47-59 (2005).

[5] V. I. Arnold, Proof of A.N. Kolmogorov's Theorem on the Preservation of Quasi-Periodic Motions under Small Perturbations of the Hamiltonian. Usp. Mat. Nauk, 18, No. 5, $13-40$ (1963). Russ. Math. Surv., 18, No. 5, 9-36 (1963).

[6] A. Ávila, R. Krikorian, Reducibility or non-uniform hyperbolicity for quasiperiodic Schrödinger cocycles, Ann. of Math., to be published (2005).

[7] R. Balescu, Hamiltonian nontwist maps for magnetic field lines with locally reversed shear in toroidal geometry. Phys. Rev. E 58, 3781-3792 (1998).

[8] J. Bricmont, K. Gawedzki, A. Kupiainen, KAM theorem and quantum field theory, Commun. Math. Phys. 201, 699-727 (1999).

[9] J. Bricmont, A. Kupiainen, A. Schenkel, Renormalization Group and the Melnikov Problem for $P D E$ 's, preprint mp_arc 01-73 (2001).

[10] J.W.S. Cassels, An introduction to Diophantine approximation, Cambridge University Press (1957).

[11] C. Chandre, Renormalization for Cubic Frequency Invariant Tori in Hamiltonian Systems with Two Degrees of Freedom, Discrete Contin. Dynam. Systems B 2, 457-465 (2002).

[12] C. Chandre, D. Farrelly and T. Uzer, Threshold to chaos and ionization for the hydrogen atom in rotating fields. Phys. Rev. A 65, 053402 1-10 (2002).

[13] C. Chandre, M. Govin, H.R. Jauslin, KAM-Renormalization Group Analysis of Stability in Hamiltonian Flows. Phys. Rev. Lett. 79, 3881-3884 (1997).

[14] C. Chandre, M. Govin, H.R. Jauslin, H. Koch, Universality for the Breakup of Invariant Tori in Hamiltonian Flows. Phys. Rev. E 57, 6612-6617 (1998).

[15] C. Chandre, H. R. Jauslin, G. Benfatto, A. Celletti, An Approximate Renormalization-Group Transformation for Hamiltonian Systems with Three Degrees of Freedom, Phys. Rev. E 60, 5412-5421, (1999). 
[16] C. Chandre and H.R. Jauslin, Renormalization-group analysis for the transition to chaos in Hamiltonian systems, Physics Reports 365, 1-64, (2002).

[17] C. Chandre, R.S. MacKay, Approximate renormalization with codimension-one fixed point for the break-up of some three-frequency tori. Phys. Lett. A 275, 394-400, (2000).

[18] P. Collet, J.-P. Eckmann, Iterated Maps on the Interval as Dynamical Systems. Birkhäuser Verlag, Basel · Boston · Berlin (1980).

[19] P. Collet, J.-P. Eckmann, H. Koch, Period doubling bifurcations for families of maps on $\mathbf{R}^{n}$, J. Stat. Phys. 25, 711-724 (1981).

[20] P. Collet, J.-P. Eckmann, H. Koch, On universality for area-preserving maps of the plane, Physica D 3, 457-467 (1981).

[21] P. Collet, J.-P. Eckmann, O.E. Lanford III, Universal properties of maps on the interval, Commun. Math. Phys. 76, 211-254 (1980).

[22] P. Coullet, C. Tresser, It'eration d'endomorphismes et group de renormalisation, J. de Physique Colloque C 539, 5-25 (1987). CRAS Paris 287 A (1987).

[23] E. de Faria, Proof of universality for critical circle mappings, Thesis, CUNY (1992).

[24] E. de Faria, W. de Melo, Rigidity of critical circle mappings I, J. Eur. Math. Soc. 1, 339-392 (1999)

[25] E. de Faria, W. de Melo, Rigidity of critical circle mappings II, Amer. Math. Soc. 13, 343-370 (2000)

[26] R. de la Llave, A tutorial on KAM theory. In "Proceedings of Symposia in Pure Mathematics" 69, A. Katok et al (eds), Amer. Math. Soc., 175-292 (2001).

[27] R. de la Llave, A. Olvera, The Obstruction criterion for non existence of invariant circles and renormalization. preprint mp_arc 03-211 (2003).

[28] D. Del-Castillo-Negrete, Dynamics and Transport in Rotating Fluids and Transition of Chaos in Area Preserving Non-twist Maps. Ph.D. Thesis, University of Texas, Austin, TX (1995).

[29] D. Del-Castillo-Negrete and P.J. Morrison, Chaotic transport by Rossby waves in shear flow. Phys. Fluids A 5, 948-965 (1993).

[30] D. Del-Castillo-Negrete, J.M. Greene, P.J. Morrison, Area Preserving Non-Twist Maps: Periodic Orbits and Transition to Chaos. Physica D 91, 1-23 (1996).

[31] D. Del-Castillo-Negrete, J.M. Greene, P.J. Morrison, Renormalization and transition to chaos in area preserving non-twist maps. Physica D 100, 311-329 (1997).

[32] A. Delshams and R. de la Llave, KAM theory and a partial justification of Greene's criterion for non-twist maps. SIAM J. Math. Anal. 31, 1235-1269 (2000).

[33] W. de Melo, S. van Strien, One-Dimensional Dynamics, Springer Verlag, New York (1993)

[34] E.I. Dinaburg, Ja. G. Sinai, The one-dimensional Schrödinger equation with quasiperiodic potential, Funkcional. Anal. i Priložen. 9, 8-21 (1975).

[35] J.-P. Eckmann, H. Epstein, On the existence of fixed points of the composition operator for circle maps, Commun. Math. Phys. 107, 213-231 (1986).

[36] J.-P. Eckmann, H. Koch, and P. Wittwer: Existence of a Fixed Point of the Doubling Transformation for Area-Preserving Maps of the Plane. Phys. Rev. A, 26, 720-722 (1982).

[37] Computer Methods and Borel Summability Applied to Feigenbaum's Equation. Lecture Notes in Physics, 227. Springer-Verlag, Berlin Heidelberg New York (1985).

[38] J.-P. Eckmann and P. Wittwer: A complete proof of the Feigenbaum conjectures. J. Stat. Phys., 46, 455-477 (1987).

[39] L.H. Eliasson, Absolutely Convergent Series Expansions for Quasi Periodic Motions. Report 2-88, Dept. of Mathematics, University of Stockholm (1988). Math. Phys. EJ, 2, No. 4, 33pp (1996).

[40] L.H. Eliasson, Floquet solutions for the 1-dimensional quasi-periodic Schrödinger equation, Commun. Math. Phys. 146, 447-482 (1992).

[41] L.H. Eliasson, Ergodic skew-systems on $\mathbb{T}^{d} \times \mathrm{SO}(3, \mathbb{R})$, Erg. Theor. Dyn. Syst. 22, 1429-1449 (2002).

[42] H. Epstein, New proofs of the existence of the Feigenbaum functions, Commun. Math. Phys. 106, 395-426 (1986).

[43] H. Epstein, Fixed Points of Composition Operators II, Nonlinearity 2, 305-310 (1989).

[44] H. Epstein, Existence and properties of p-tupling fixed points, Commun. Math. Phys 215, 443-476 (2000).

[45] H. Epstein, J. Lascoux, Analyticity properties of the Feigenbaum function, Commun. Math. Phys 81, 437-453 (1981).

[46] A. Epstein, M. Yampolsky, The universal parabolic map, Ergodic Theory and Dynam. Systems, to appear. 
[47] D.F. Escande, Stochasticity in Hamiltonian Systems: Universal Aspects, Phys. Rep. 121, $165-261$ (1985).

[48] D.F. Escande, F. Doveil, Renormalisation Method for Computing the Threshold of the Large Scale Stochastic Instability in Two Degree of Freedom Hamiltonian Systems. J. Stat. Phys. 26, $257-284$ (1981).

[49] M.J. Feigenbaum, Quantitative universality for a class of nonlinear transformations, J. Stat. Phys. 19, 25-52 (1978).

[50] M.J. Feigenbaum, Universal metric properties of non-linear transformations, J. Stat. Phys. 21, 669-706 (1979)

[52] M.J. Feigenbaum, L.P. Kadanoff, S.J. Shenker, Quasi-Periodicity in Dissipative Systems: A Renormalization Group Analysis. Physica D 5, 370-386 (1982).

[51] J. Feldman, E. Trubowitz, Renormalization in Classical Mechanics and Many Body Quantum Field Theory. Journal d'Analyse Mathématique, 58, 213-247 (1992).

[53] A. Fink, Almost periodic differential equations, Lecture Notes in Mathematics, 377, Springer Verlag, Berlin · Heidelberg · New York (1974).

[54] D.G. Gaidashev, Renormalization of isoenergetically degenerate Hamiltonian flows and associated bifurcations of invariant tori, Discrete Contin. Dynam. Systems A 13, 63-102 (2005).

[55] D.G. Gaidashev, H. Koch, Renormalization and Shearless Invariant Tori: Numerical Results, Nonlinearity 17,1713-1722 (2004).

[56] D.G. Gaidashev, M. Yampolsky, Cylinder renormalization of Siegel disks, preprint mp_arc 06-43 (2006).

[57] G. Gallavotti, Perturbation Theory. In "Mathematical Physics towards the XXI Century", R. Sen, A. Gersten (eds), Ben Gurion University Press, Ber Sheva, 275-294 (1994).

[58] G. Gallavotti, G. Gentile, V. Mastropietro, Field theory and KAM tori. Math. Phys. EJ, 1, No. 5, 13pp (1995).

[59] G. Gentile, V. Mastropietro, Tree Expansion and Multiscale Decomposition for KAM Tori. Nonlinearity, 8, 1-20 (1995).

[60] M. Govin, C. Chandre, H.R. Jauslin, KAM-Renormalization Group Approach to the Breakup of Invariant Tori in Hamiltonian Systems. Phys. Rev. E 57, 1536-1543 (1998).

[61] A. Haro, An algorithm to generate canonical transformations: application to normal forms, Physica D 167, 197-217 (2002).

[62] E. Hille, R.S. Phillips, Functional analysis and semi-groups, AMS Colloquium Publications, 31 (1974).

[63] B. Hinkle, Parabolic limits of renormalization, Ergodic Theory Dynam. Systems 20, 173-229 (2000).

[64] M.W. Hirsch, C.C. Pugh, M. Shub, Invariant Manifolds. Lecture Notes in Mathematics, Volume 583, Springer Verlag, Berlin · Heidelberg · New York (1977).

[65] W. Horton, H.-B. Park, J.-M. Kwon, D. Strozzi, P.J. Morrison and D.-I. Choi, Drift wave particle transport in reversed shear profile. Phys. Plasma 5, 3910-3917 (1998).

[66] R. Johnson, J. Moser, The rotation number for almost periodic potentials, Commun. Math. Phys. 84, 403-438 (1982).

[67] L. Jonker, D. Rand, Universal properties of maps of the circle with $\varepsilon$-singularities, Commun. Math. Phys 90, 273-292 (1983).

[68] L.P. Kadanoff, Scaling for a critical Kolmogorov-Arnold-Moser trajectory. Phys. Rev. Lett. 47, 1641-1643 (1981).

[69] J.A. Ketoja and R.S. MacKay, Fractal Boundary for the Existence of Invariant Circles for AreaPreserving Maps: Observations and Renormalisation Explanation, Physica D 35, 318-334 (1989).

[70] K. Khanin, J. Lopes Dias, J. Marklof, Multidimensional continued fractions, dynamical renormalization and KAM theory, preprint mp_arc 05-304 (2005).

[71] K. Khanin, Ya.G. Sinai, The Renormalization Group Method and KAM Theory. In "Nonlinear Phenomena in Plasma Physics and Hydrodynamics", R.Z. Sagdeev (ed), Mir, 93-118 (1986).

[72] K. Khanin, Ya.G. Sinai, Renormalization Group Methods in the Theory of Dynamical Systems. Int. J. Mod. Phys., B2, 147-165 (1988).

[73] D.Y. Kleinbock, G.A. Margulis, Flows on homogeneous spaces and Diophantine approximation on manifolds, Ann. of Math. (2) 148, 339-360 (1998).

[74] H. Koch, A renormalization group for Hamiltonians, with applications to KAM tori, Erg. Theor. Dyn. Syst. 19, 1-47 (1999).

[75] H. Koch, On the renormalization of Hamiltonian flows, and critical invariant tori. Discrete Contin. Dynam. Systems A, 8, 633-646 (2002). 
[76] H. Koch, A Renormalization Group Fixed Point Associated with the Breakup of Golden Invariant Tori. Discrete Contin. Dynam. Systems 11, 881-909 (2004).

[77] H. Koch, Existence of Critical Invariant Tori, Erg. Theor. Dyn. Syst., to appear.

[78] H. Koch, S. Kocić, work in progress.

[79] H. Koch, J. Lopes Dias, Renormalization of Diophantine skew flows, with applications to the reducibility problem, preprint mp_arc 05-285 (2005).

[80] H. Koch, A. Schenkel and P. Wittwer, Computer-Assisted Proofs in Analysis and Programming in Logic: A Case Study, SIAM Rev. 38, 565-604 (1996).

[81] S. Kocić, Renormalization of Hamiltonians for Diophantine frequency vectors and KAM tori, Nonlinearity 18, 2513-2544 (2005).

[82] A. N. Kolmogorov, On Conservation of Conditionally Periodic Motions Under Small Perturbations of the Hamiltonian. Dokl. Akad. Nauka SSSR, 98, 527-530 (1954).

[83] D. Kosygin, Multidimensional KAM Theory from the Renormalization Group Viewpoint. In "Dynamical Systems and Statistical Mechanics", Ya.G. Sinai (ed), AMS, Adv. Sov. Math., 3, 99-129 (1991).

[84] R. Krikorian, Réductibilité des systèmes produits-croisés à valeurs dans des groupes compacts, Astérisque 259, vi+216 (1999).

[85] R. Krikorian, Réductibilité presque partout des flots fibrés quasi-périodiques à valeurs dans des groupes compacts, Ann. Sci. École Norm. Sup. (4) 32, 187-240 (1999).

[86] R. Krikorian, $C^{0}$-densité globale des systèmes produits-croisés sur le cercle réductibles, Erg. Theor. Dyn. Syst. 19, 61-100 (1999).

[87] R. Krikorian, Global density of reducible quasi-periodic cocycles on $\mathbf{T}^{1} \times \mathrm{SU}(2)$, Ann. of Math. (2) 154, 269-326 (2001).

[88] W.T. Kyner, Rigorous and formal stability of orbits about an oblate planet. Mem. Am. Math. Soc. 81, 1-27 (1968).

[89] J.C. Lagarias, Geodesic multidimensional continued fractions, Proc. London Math. Soc. 69, 464-488 (1994).

[90] O.E. Lanford, Remarks on the accumulation of period-doubling bifurcations, In: Mathematical Problems in Theoretical Physics. Lecture Notes in Physics, 116, 340-342 (1979).

[91] O.E. Lanford, Computer-Assisted Proof of the Feigenbaum Conjectures, Bull. A.M.S. (New Series), 6, 427-434 (1982).

[92] O.E. Lanford, A Shorter Proof of the Existence of the Feigenbaum Fixed Point, Commun. Math. Phys., 96, 521-538 (1984).

[93] O.E. Lanford, Renormalization group methods for critical circle mappings with general rotation number. In: VIII-th International Congress on Mathematical Physics (Marseille, 1986), Wordl Sci. Publishing, Singapore, 532-536 (1987)

[94] J. Lopes Dias, Renormalisation of flows on the multidimensional torus close to a KT frequency vector, Nonlinearity 15, 647-664, (2002).

[95] J. Lopes Dias, Renormalisation scheme for vector fields on $T^{2}$ with a Diophantine frequency, Nonlinearity 15, 665-679, (2002).

[96] J. Lopes Dias, Brjuno condition and renormalization for Poincaré flows, preprint (2004).

[97] J. Lopes Dias, Renormalization and reducibility of Brjuno skew-systems, preprint (2004).

[98] M. Lyubich, Renormalization ideas in conformal dynamics, In: Cambridge Seminar "Current Developments in Mathematics", May 1995, International Press, Cambridge MA, 155-184 (1995).

[99] M. Lyubich, Feigenbaum-Coullet-Tresser Universality and Milnor's Hairiness Conjecture, Annals of Mathematics 149, 319-420 (1999).

[100] R.S. MacKay, Renormalisation in Area Preserving Maps. Thesis, Princeton (1982). World Scientific, London (1993).

[101] R.S. MacKay, Renormalisation Approach to Invariant Circles in Area-Preserving Maps, Physica D 7 (1983), 283-300.

[102] R.S. MacKay, Three Topics in Hamiltonian Dynamics. Preprint U. Warwick (1994). Also in: "Dynamical Systems and Chaos", Vol.2, Y. Aizawa, S. Saito, K. Shiraiwa (eds), World Scientific, London (1995).

[103] R.S. MacKay, J.D. Meiss, J. Stark, An Approximate Renormalization for the Break-up of Invariant Tori with Three Frequencies. Phys. Lett. A 190, 417-424 (1994).

[104] M. Martens, The periodic points of renormalization, Ann. Math. 147, 543-584 (1998).

[105] C. McMullen, Complex Dynamics and Renormalization. Annals of Mathematics Studies 135, Princeton Univ. Press (1994). 
[106] C. McMullen, Renormalization and 3-Manifolds which Fiber over the Circle. Annals of Mathematics Studies 142, Princeton Univ. Press (1996).

[107] A. Mehr, D.F. Escande, Destruction of KAM Tori in Hamiltonian Systems: Link with the Destabilization of nearby Cycles and Calculation of Residues. Physica D 13, 302-338 (1984).

[108] B.D. Mestel, A computer assisted proof of universality for cubic critical maps of the circle with golden mean rotation number. Ph.D. Thesis, Math. Dept., University of Warwick (1985).

[109] B.D. Mestel, A. Osbaldestin, Feigenbaum theory for unimodal maps with asymmetric critical point: rigorous results, Commun. Math. Phys. 197, 211-228 (1998).

[110] J. Moser, On Invariant Curves of Area-Preserving Mappings of an Annulus. Nachr. Akad. Wiss. Gött., II. Math. Phys. Kl 1962, 1-20 (1962).

[111] J. Moser, J. Pöschel, An extension of a result by Dinaburg and Sină on quasiperiodic potentials, Comment. Math. Helv. 59, 39-85 (1984).

[112] S. Ostlund, D.A. Rand, J. Sethna, E. Siggia, Universal Properties of the Transition from Quasiperiodicity to Chaos in Dissipative Systems. Physica D 8, 303-342 (1983).

[113] J. Palis, W. de Melo, Geometric Theory of Dynamical Systems. An Introduction. Springer-Verlag, Berlin · New York (1982).

[114] D.A. Rand, Existence, Non-Existence and Universal Breakup of Dissipative Golden Invariant Tori. Nonlinearity, 5, 639-706 (1992).

[115] M. Rychlik, Renormalization of cocycles and linear ODE with almost-periodic coefficients, Invent. Math. 110, 173-206 (1992).

[116] S.J. Shenker, L.P. Kadanoff, Critical behavior of a KAM surface. I. Empirical results. J. Stat. Phys. 27, 631-656 (1982).

[117] A. Stirnemann, Towards an Existence Proof of MacKay's Fixed Point. Comm. Math. Phys. 188, $723-735$ (1997).

[118] E.C.G. Stueckelberg, A. Petermann, La normalisation des constantes dans la theorie des quanta, Helv. Phys. Acta 26, 499-520 (1953).

[119] D. Sullivan, Quasiconformal homeomorphisms and dynamics, topology and geometry. In: Proc. ICM-86 Berkeley II, AMS, Providence RI, 1216-1228 (1987).

[120]. Sullivan, Bounds, quadratic differentials, and renormalization conjectures. In: AMS Centennial Publications II, Mathematics into Twenty-first Century, 417-466 (1992).

[121] W. Thirring, A Course in Mathematical Physics I: Classical Dynamical Systems. Springer-Verlag, Berlin · New York · Wien (1978).

[122] S. Tompaidis, Approximation of Invariant Surfaces by Periodic Orbits in High-Dimensional Maps: some Rigorous Results. Experimental Math. 5, 197-209 (1996).

[123] J. Wilbrink, New Fixed Point of the Renormalisation Operator Associated with the Recurrence of Invariant Circles in Generic Hamiltonian Maps, Nonlinearity 3, 567-584, (1990).

[124] M. Yampolsky, Complex bounds for renormalization of critical circle maps, Ergodic Theor. Dyn. Syst. 19, 227-257 (1999).

[125] M. Yampolsky, Hyperbolicity of renormalization of critical circle maps, Publ. Math. Inst. Hautes Etudes Sci. 96, 1-41 (2002).

[126] M. Yampolsky, Global renormalization horseshoe for critical circle maps, Commun. Math. Phys. 240, 75-96 (2003).

[127] M. Yampolsky, On the eigenvalues of a renormalization operator, Nonlinearity 16, 1565-1571 (2003).

[128] References $[18,19,20,21,22,33,36,37,38,43,42,45,44,49,50,63,92,91,90,98,99,105,109$, $119,120, \ldots]$.

[129] References [3, 4, 24, 25, 23, 33, 31, 30, 29, 28, 35, 46, 52, 63, 67, 68, 69, 93, 98, 101, 100, 103, 104, $105,106,108,112,114,117,123,124,126,127,125, \ldots]$. 